\title{
FINITE ELEMENT INVESTIGATION OF THERMO-ELASTIC AND THERMO-PLASTIC CONSOLIDATION
}

\author{
By \\ B. L. Aboustit \\ January 1984
}

Work Performed Under Contract No.: DE-AS20-82LC10886

For

U. S. Department of Energy

Office of Fossil Energy

Morgantown Energy Technology Center

Laramie Project Office

Laramie, Wyoming

By

Ohio State University

Columbus, Ohio

Technical Information Center

Office of Scientific and Technical Information United States Department of Energy 


\section{DISCLAIMER}

This report was prepared as an account of work sponsored by an agency of the United States Government. Neither the United States Government nor any agency Thereof, nor any of their employees, makes any warranty, express or implied, or assumes any legal liability or responsibility for the accuracy, completeness, or usefulness of any information, apparatus, product, or process disclosed, or represents that its use would not infringe privately owned rights. Reference herein to any specific commercial product, process, or service by trade name, trademark, manufacturer, or otherwise does not necessarily constitute or imply its endorsement, recommendation, or favoring by the United States Government or any agency thereof. The views and opinions of authors expressed herein do not necessarily state or reflect those of the United States Government or any agency thereof. 


\section{DISCLAIMER}

Portions of this document may be illegible in electronic image products. Images are produced from the best available original document. 


\title{
DISCLAIMER
}

\begin{abstract}
This report was prepared as an account of work sponsored by an agency of the United States Government. Neither the United States Government nor any agency thcreof, nor any of their employees, makes any warranty, express or implied, or assumes any legal liability or responsibility for the accuracy, completeness, or usefulness of any information, apparatus, product, or process disclosed, or represents that its use would not infringe privately owned rights. Reference herein to any specific commercial product, process, or service by trade name, trademark, manufacturer, or otherwise does not necessarily constitute or imply its endorsement, recommendation, or favoring by the United States Government or any agency thereof. The views and opinions of authors expressed herein do not necessarily state or reflect those of the United States Government or any agency thereof.
\end{abstract}

This report has been reproduced directly from the best available copy.

Available from the National Technical Information Service, U. S. Department of Commerce, Springfield, Virginia 22161.

Price: Printed Copy A07

Microfiche An1

Codes are used for pricing all publications. The code is determined by the number of pages in the publication. Information pertaining to the pricing codes can be found in the current issues of the following publications, which are generally available in most libraries: Energy Reseurch Abstracts (ERA); Government Reports Announcements and Index (GRA and I); Scientific and Technical Abstract Reports (STAR); and publication NTIS-PK-360 available from NTIS at the above address. 


\title{
FINITE ELEMENT INVESTIGATION OF THERMO-ELASTIC AND THERMO-PLASTIC CONSOLIDATION
}

\author{
By \\ B. L. Aboustit
}

January 1984

Work Performed Under Contract No.: DE-AS20-82LC10886

\author{
For \\ U. S. Department of Energy \\ Office of Fossil Energy \\ Morgantowu Eutigy Technology Center \\ Laramie Project Office \\ Laramie, Wyoming 82071
}

By

Ohio State University

Columbus, Ohio 43210 


\section{THIS PAGE}

\section{WAS INTENTIONALLY \\ LEFT BLANK}


TABLE OF CONTENTS

Page

LIST OF TABLES .................. $v$

LIST OF FIGURES ...................... vi

NOMENCLATURE ................................ vii

ABSTRACT ........................... $x^{i}$

Chapter

I. INTRODUCTION ..................... 1

1.1 General Remarks. . . . . . . . . 1

1.2 Review of Literature ......... 2

1.3 Objectives and Scope.......... 9

II. THERMAL CONSOLIDATION - AN ELASTIC

ANALYSIS ..................... 11

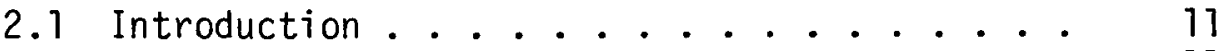

2.2 Batance Equations. . . . . . . . . 11

2.2.1 Conservation of Mass ....... 12

2.2.2 Conservation of Linear Momentum 12

2.2.3 Total Stress Tensor ........ 13

2.2.4 Diffusive Resistance........ 13

2.3 Constitutive Equations .......... 14

2.3.1 Darcy's Law. ........... . 16

2.3.2 Continuity Equation. ...... . 17

2.3.3 Fourier's Law. . . . . . . . . . 18

2.3.4 Energy Equation. . . . . . . . 18

2.4 Variational Formulation. . . . . . . . 19

2.4 .1 Generàl. . . . . . . . . . 19

2.4.2 The Initial Boundary Value Problem of Thermoelastic Consolidation .... 21

2.4.3 Alternative Forms for Continuity and Energy Equations........ 24

2.4.5 Extended Variational Principle.... . 26

2.4.6 Specializations ........ . 32

2.5 Finite Element Formulations. . . . . . 33

2.5.1 Spatial Discretization ....... 33

2.5.2 Temporal D1scret1zation....... 36

2.5.3 Choice of Finite Elements. . . . . . 37 
III. THERMAL CONSOLIDATION - AN ELASTOPLASTIC

ANALYSIS ...................... 41

3.1 Introduction ............ 41

3.2 Plastic Strain Increment. . . . . . . . 42

3.3 Stress Strain Relation. ......... 45

3.4 Field Equations in Incremental Form . . . 48

3.5 Finite Element rormulation. ....... 50

3.5 .1 Incremental Scheme. . . . . . . 50

3.5.2 Incremental Interative Scheme .... 51

IV. NUMERICAL RESULTS. .................. 54

4.1 Introduction. . . . . . . . . 54

4.2 Code Validation ............ . . 54

4.2.1 Elastic Anarysis. ........ . 54

4.2.2 Efficiency of the Discretization

Schemes ........... 57

4.2.3 Elastoplastic Analysis. . . . . . . 60

4.3 Field Application ........... 62

V. CONCLUSIONS ................. 81

5.1 Preliminary Remarks . . . . . . . . 81

5.2 Concluding Remarks. . . . . . . . . . 81

5.3 Applications............ 83

5.4 Research Recommendations........ 84

REFERENCES

$\begin{array}{ll}\text { APPENDICES } & 91\end{array}$

A. Classical Yield Criteria and Alternative ................... yi

B. Yield Surface with an Expanding Cap ....... 101

C. Incremental Stress Strain Relation in

Plane Strain............ 108

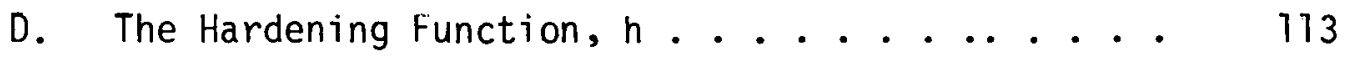

E. Numerical Procedure for Evaluating Stress
Increment . . . . . . . . . . 117

F. Proof of Theorem I . . . . . . . . 127 


\section{LIST OF TABLES}

Table 4.1 Vertical surface settlement History

Page Using $=.875$ for the $8-8-8$ and the 8-4-4 Element. . . . . . . . . 64

Table 4.2 Material Properties for Centralia Coal Gasification Site . . . . . . 65

Table A-1 Definition of C constants . . . . . 98 


\section{LIST OF FIGURES}

2.1 The 8-4-4 Isoparametric Element......

2.2 The 8-8-8 Isoparametric Element......

4.1 One-Dimensional Thermoelastic Consolidation . . . . . . . . . . . 66

4.2 Mesh for the 8-4-4 and the 8-8-8 Elements. 67

4. 3 Pressure and Temperature Profiles at Two Different Time Steps . . . . . . . . 68

4.4 Surface Settlement History for Isothermal and l'hermal consolidation . . . . . . .

4.5a through 4.5h Effect of Spatial Discretization on Pressure and Temperature Profiles.

4.6a through 4.6c Effect of Temporal Partitioning on Pressure and Temperature Profiles. . .

4.7 'Thick Circular Cylinder Subjected to Internal Pressure . . . . . . . . . .

4.8 Inner Surface Displacement with Increasing Pressure . . . . . . . . .

4.9 Surface Settlement History and Progression of Plastic Zones. . . . . . . . . . . .

4.10a through 4.10h Elastic and Elasto-plastic Pressure and Temperature Profiles . . . .

4.11 Finite Element Mesh and Strata Description for Centralia Coal Gasification Site. . .

4.12 Cavity Temperature versus Time. . . . . .

1.13 Surfaoe settloment History and Progrcsion of Plastic Zones, Centralia Coal Gasification (Hatched area implies yielded element.) . .

A.1 Mohr-Coulomb and Tresca Yield Criteria. .

A.2 Drucker-Prager and Von Mises Yield Criteria 
A. $3 \pi$-Plane Representation of Tresca, Von Mises, Mohr-Coulomb and Drucker-Prager Yield Criteria................. . . 100

B.l Elliptic Cap Model . . . . . . . . . 106

B.2 Response of Idealized soil to Hydrostatic Stress . . . . . . . . . . . . .

E.l Incremental Stress Changes at a Point in an Elasto-Plastic Continuum at Initial Yield. .

E.2 Incremental Stress Changes in an Already Yielded Point in an Elasto-Plastic Continuum

E.3 Secant Stiffness Method. . . . . . . . .

E.4 Multi-Step Tangent Stiffness Method with Scaling. . . . . . . . . . . . . . .

E.5 The Incremental Iterative Scheme . . . . 


\section{NOMENCLATURE}

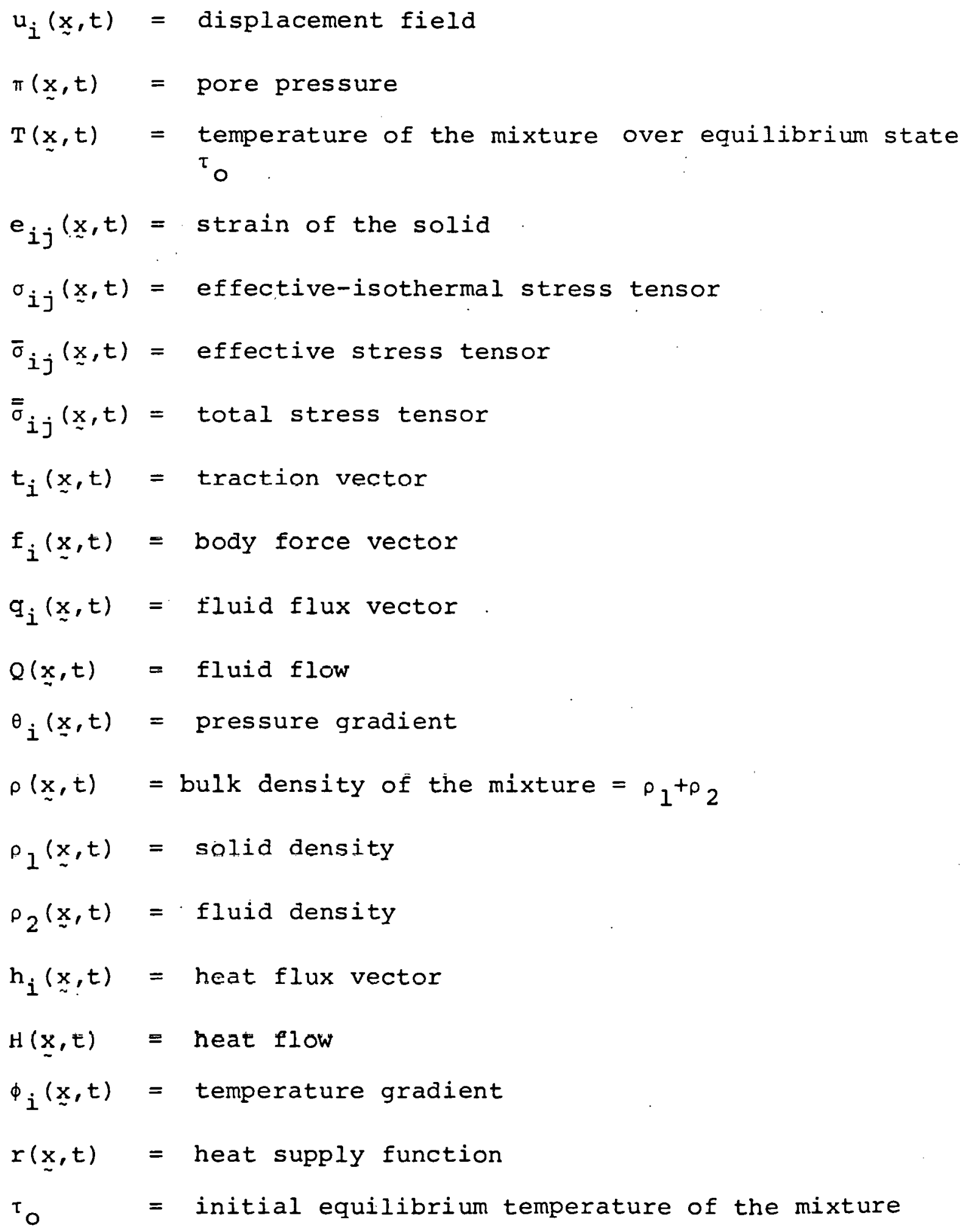




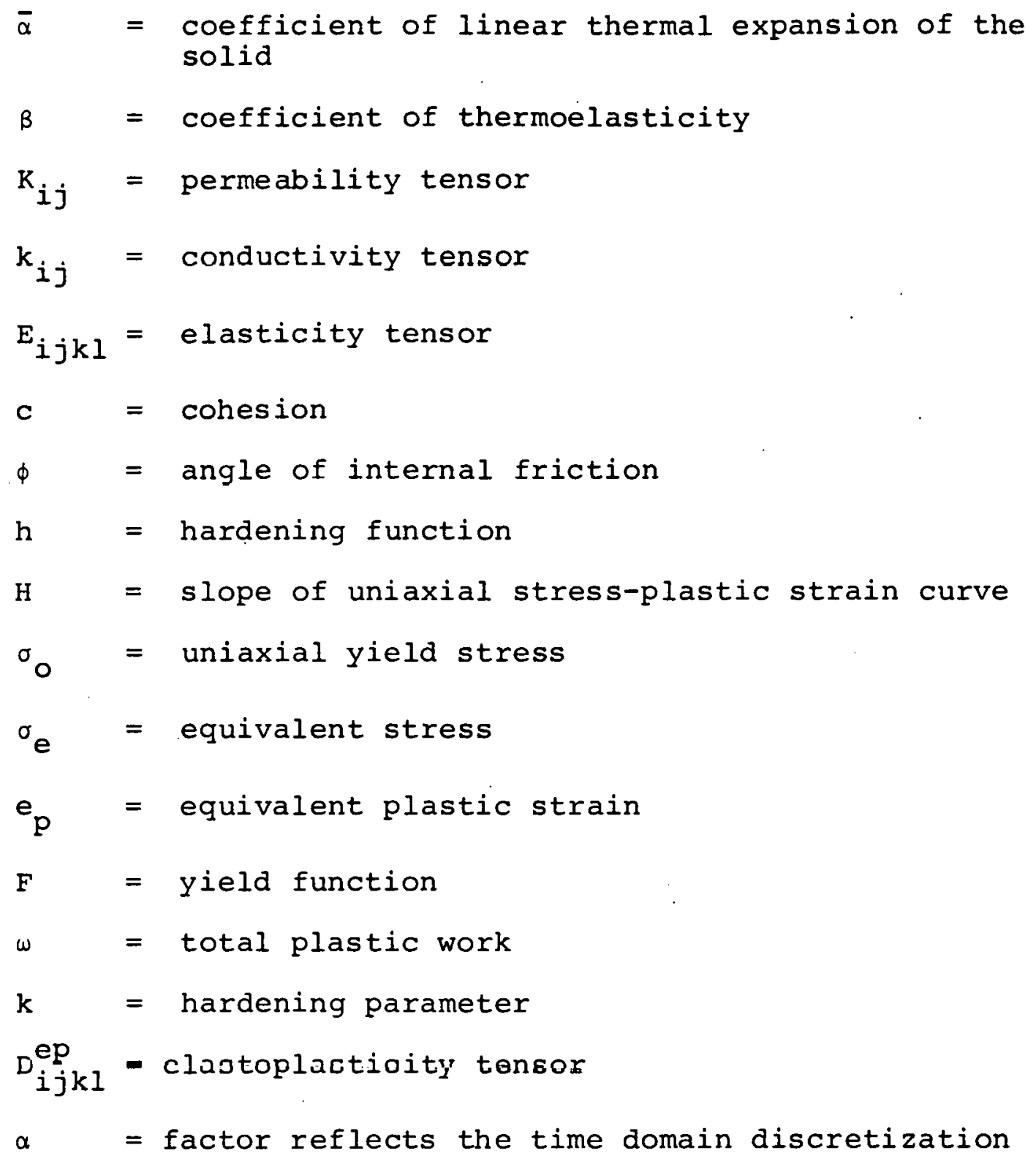


THIS PAGE

\section{WAS INTENTIONALLY \\ LEFT BLANK}




\section{FIIITE ELENENT INVESTIGATIONS OF THERMO-ELASTIC}

AIND THERNAC-EDASTIC CONSOLIDATION

By:

Baher Iabeeb Aboustit, Ph.D.

The Ohio State University, 1983

The transient response of saturated continua due to thermal as well as mechanical loads is investigated in both elastic and plastic ranges.

When the two phase saturated meaia are subjectec to thermomechanical loacing, the energy equation is couplec with the mass flow ari solid deformation equations resulting in the initial boundary value problem of thermal consolidation. The solid behavior may be assumed to be either elastic or elastoplastic leading to the associated theorice of thermuelastic and thermoelastoplastic consolidation. Numerous solutions to isothermal consolidation problens ate avaliable in the iiterature, but thermal consolidation response solutions are not available.

The governing equations for the quasi-static infinitesimal theory of thezmoelastic consolidation are developed by using the theory ce mixtures. An equivalent variational principle is acvelogs 
formulations. Two isoparanetric elements of the composite type are employed for the spatial discretization. The formulation is extenced to the plastic ranges by modeling the solid phase as an elastic work hardening material with an associatę flow rule. An incremental iterative scheme is developed to solve this nonlinear transient problem. Several special purpose computer codes are developed for evaluating the isothermal, thermal, elastic and elastoplastic plane strain consolidation responses. These codes have been evaluated against limiting cases available in the literature. The effects of temporal and spatial interpolation schemes are investigated for oncdimensional thermoelastic consolidation problems. An application dealing with a plane strain underground coal gasification problem is also presented.

Although the analysis is restricted to the infinitesimal theory of deformations, second ordcr geometris effects can be inciuded in the andysis: Fluid compressibility and a direct coupling between mass and fluid flow can alsu be considered. The incorporation of the effects of stress and temperature dependent permeabilities, conductivities and yield functions is recommended for future work. Aithough only geotechnical applications have been investigated, the analysis is also generally applicable to polymer and composite hygrothermal material response evaluation. 
CHAPTER I

INTRODUCTION

\subsection{General Remarks}

The problem of fluid flow through porous media has received considerable attention in many engineering applications including foundation analysis, reservoir flow/depletion, energy resource extraction, and nuclear waste interaction.

When saturated clay is subjected to an external loading, water drains gradually out of the pores and the solid skeleton deforms instantaneously. This transient coupled phenomenon is known as consolidation.

In some situations, saturated soils are subjected to merhanical as well as thermal loading such as in situ coal gasification, geothermal energy recovery, hydraulic fracturing, and nuclear waste management. This increases the complexity of the problem, since the transient thermal flow problem is coupled with the consolidation problem. This phenomenon is called thermal consolidation.

Generalization of Biot's isothermal theory of consolidation to include thermal effects is alfficult becaues it is based on phenomenological assumptions. The theory of mixtures provides an excellent basis for deriving thermal and 
isothermal theories of consolidation. For thermoelastic consolidation, the soil or rock medium is assumed to be elastic. However, experiments have demonstrated that geotechnical materials are strongly nonlinear and can be modeled as elastoplastic materials. This constitutive characterization leads to thermo-elasto-plastic consolidation theories.

Analytical solutions for this complex phenomenon, even for simple thermoelastic one-dimensional problems, are not available in the literature. For thermo-plastic probleils, closed form analytical representation of the solutions is impossible because of the inherent non-linearities. This suggests the use of the finite element method for solving such nonlinear and transient problems. with complex geometries, layering conditions and boundary conditions.

\subsection{Review of Literature}

The subject of flow through porous media has been frequently dealt with in the literature. Scheidegger [54] and Polubarinova-Kochina [41] have given an excellent review on this subject. For a one-dimensional rluw of fluid through an undeformabile porous solid, Darcy observed a linear relationship between fluid velocity and the potential causing the flow, where the constant of proportionality is the permeability coefficient of the porous media. In general, the permeability coefficient depends on the size, shape, distribution of the pores, temperature and fluid velocity. For three-dimensional flow through porous media; Darcy's law is 
also used by extending the permeability coefficient to a third order tensor. Analytical and numerical solutions to the problem of flow through a incompressible porous media, which use Darcy's law as a basic equation can be found in many publications, e.g. Scheidegger [54], Pluborinova-Kochina [41] and Desai [17] .

Terzaghi [64] used both experimental and analytical methods to consider the deformation of the solid skeleton. In 1923, Terzaghi developed the one-dimensional theory of consolidation with the following assumptions: (1) the soil is completely saturated with water; (2) the soil particles and the pore water are incompressible; (3) the flow of fluid obeys Darcy's law; (4) the solid skeleton is linearly elastic in terms of effective stresses; (5) homogeneous soil properties; (6) small strains and small displacements. For a column of soil subjected to constant vertical loading, the equation governing the transient phenomenon of consolidation, is the same as the well known diffusion equation in engineering mechanics. The definition of Terzaghi for the effective stress agrees with the modern theory of consolidation [48]. The effective stress is defined as the difference between the total stress acting on the soil sample and the pore pressure. This theory is limited to cases where the soil is subjected to constant loading extended over a relatively large area. Subsequent generalizations and extensions of the theory can be found in the literature (cf. [56] for a review). 
Biot $[2,3,4,5,6]$ assuming the existence of strain energy for the fluid solid mixture, postulated constitutive relations for the three-dimensional theory of consolidation. The solid is assumed to be linearly elastic and isotropic. The fluid flow obeys Darcy's law and the fluid pressure is assumed to act only over the pore space. Generalizations to anisotropic and viscoelastic solids are also considered. Biot's consolidation theory has been criticized by Sandhu [48]. The theory involves the dependency of the pore pressure on the solid deformation which contradicts Darcy's law.

Green and Naghdi [23] proposed a dynamical theory of interacting continua, based on the energy balance equation employing invariance conditions under superposed rigid body motion and entropy production inequality. Crochet and Nagdhi [15] used the dynamical theory of interacting continua to present governing equations for fluid flow through a porous elastic solid.

Sandhu [48] used the work of Green and Naqhdi $[231$ and Crochet and Nagdhi [15] to derive the field equations for the initial boundary value problem of linear elastic quasi-static isothermal consolidation. Sandhu [48] and Sandhu and wilson [49] presented a variational principle and associated finite element formulation. A composite six noded triangular element is developed for the spatial discretization, wherein the displacement is assigned at all the nodes (i.e., quadratic 
displacement), while the pore pressure is assigned to the corner nodes only (i.e., linear pressure). A linear interpolation as well as logarthmic interpolation is used in the time domain discretization.

Hwang et al. [25] proposed a different logarthmic interpolation for the temporal discretization. In this scheme, the time integration factor, $\alpha$, depends on the current value of the time variable, which results in an expensive equation solving process for constant time increments. Yooko et al. $[67,68,69]$ employed eight noded isoparametric elements with a discontinuous pressure field to allow for the jump conditions on boundaries with specified tractions, which resulted in good solutions to the undrained problem. However, at subsequent time steps it gave poor approximations.

In the displacement interpolation, Christian and Boehmer [14] used a quadrilateral element composed of four constant strain triangles with the displacement of the central node eliminated by static condensation. The pressure is assumed to be constant throughout the element, and the value of the pressure at the center of the element and its four surrounding elements was used to evaluate the quadratic variation of the pore pressure. This procedure is based on equating the rate of outflow and the reduction in the solid volume. The scheme is explicit and is subjected to stability conditions.

Ghaboussi and Wilson [21] extended the formulation by Sandhu and wilson [49] to account for fluid compressibility 
using Biot's assumption [4] of the existence of the strain energy function for the mixture. Four noded isoparametric elements were used for both the displacement and pore pressure. However, an additional incompatible mode was included in the displacement interpolation. Smith and Hobbs [63] used the same element of Ghaboussi and Wilson [21] but without the incompatible displacement modes. Inaccurate results were observed especially near the impervious strata. Booker and Small [8] investigated the stahility of the time domain discretization for the consolidation equations. It was found that for the integration factor, $\alpha \geq .5$, the scheme is unconditionally stable. Krause [28] used the virtual work principle to formulate the finite element equations for the isothermal consolidation problem. Two different schemes were suggested to reduce the number of degrees of freedom, either by eliminating the pressure or the displacement. However, the resulting matrices are no longer banded as they were in the complete formulation.

Sandhu [51] presented general variational principles equivalent to the direct, the complementary and the mixed forms for the field equations governing the isothermal consolidation. Generalizations to nonlinear constitutive laws were also proposed. Sandhu [53] presented an eight noded isoparametric element in which the displacements are assigned to all the nodes and the pore pressure is assigined at the corner nodes only: This element is referred to as the 8-4 
element. Numerical comparisons were conducted using this element, and the element proposed by Yooko et al. (8-8 element) and Sandhu and Wilson's [49] element (6-3 element). The 6-3 element exhibits a directional bias, although for a symmetric mesh it gives good results. The 8-4 element was found to be superior to the 8-8 element. Oscillatory pressure responses were obtained when the 8-8 element is used with $\alpha=0.5$.

Sharabi [59] used the theory of mixtures [15,23] to write the governing equations for the isothermal consolidation problem allowing for finite deformations, nonlinear elastic soil properties and variable permeability. The Galerkin method is used to derive finite element equations in a linearized incremental form. A five noded quadrilateral element is used for the displacement with constant shear strain and the pore pressure is assigned at the corner nodes only. Reasonably good results are obtained in the linear analysis.

Small, Booker and Davis [62] used the initial stress method in plastic analysis [70] to obtain an incremental iterative scheme for the isothermal elastoplastic consolidation for a solid skeleton obeying the Mohr-Coulomb yield criterion with a non-associated flow rule. Carter, Small and Booker [10] presented the theory and finite element formulations for finite elastic isothermal consolidation, using an Eulerian description. These authors [11] combined their previous work 
$[10,62]$ to present an elastoplastic analysis with finite deformation.

Prevost $[43,44]$ used an incremental scheme for solving the isothermal consolidation problem using the four noded element of Smith et al. $\lceil 631$ but with a different numbcr of Gaussian points for the solid and the coupling matrices. Siriwardan and Desai. [61] presented two numerical schemes for isothermal elastoplastic consolidation. The two schemes are of an incremental nature, the first one uses the residual load approach and the second one utilizes the tangential stiffness approach.

Schiffman [55] used the theory of mixtures [15,23] along with Biot's theory [4] to write the field equations for the thermoelastic consolidation theory. Onsager's principle [20] is used to define the coupling between hoat and fluid flow. Witherspoon et al. [65] have presented field equations for the thermuelastic consolidation similar to those previously presented by Schiffman [55]. In their Galerkin-type finite element formulation, the temperature was added "physieally" to the functional developed earlier by Ghaboussi and Wilson [22] for the poroelastic component of the hydrothermoelastic phenomenon.

It is worth noting that Biot's theory, with its drawback listed earlier, has been the primary basis for formulating the thermoelastic consolidation equations in Refs.[55, $65]$. 


\subsection{Objectives and Scope}

The purpose of this work is to study the transient behavior of saturated soils subjected to both thermal and mechanical loads in both elastic and plastic ranges.

In this study, the governing equations for the quasistatic, infinitesimal theory of thermoelastic consolidation are developed using the theory of mixtures $[15,23]$ and the isothermal consolidation theory [48]. A variational principle equivalent to the thermoelastic consolidation field equations, initial and boundary conditions is developed, along with associated finite element formulation. Two different isoparametric elements are suggested. The work is then extended to the elastoplastic case. An incremental iterative scheme is used to obtain the solution.

In Chapter II, the governing equations for thermoelastic consolidation are obtained following the approach of Green and Naqdhi [23] and Crochet and Naghdi [15] for the theory of mixtures and Sandhu [48] for the isothermal consolidation. A general variational principle is obtained based on the approach of Sandhu and Pister [50]. The finite element method is used to develop numerical procedures for the solution. Two types of isoparametric elements are utilized.

In Chapter III, the formulation is extended to the plastic range by adopting several isothermal yield criteria with an associated flow rule. An incremental iterative scheme is used for the solution. 
Applications are demonstrated in Chapter IV. Thermoelastic and thermoelastoplastic conslidation of a bounded column of saturated clay are first considered. Results for the elastic analysis are compared with the analytical solution of Terzaghi and numerical solutions of Sandhu [53] for the isothermal consolidation problem and the temperature profile is compared with the analytical solution of Carslaw and Jaeger [9]. Computational efficiencies of the two elements are discussed. Field simulation pertaining to underground coal conversion site are also conducted.

Conclusions and a summary of the investigations are presented in Chapter $V$. Future research recommendations are also discussed. Detailed computational procedures and supporting theories are discussed in the Appendices. 
CHAPTER II

THERMAL CONSOLIDATION - AN ELASTIC ANALYSIS

\subsection{Introduction}

In this chapter, the field equations for flow of an incompressible fluid through a linearly elastic solid subjected to both thermal and mechanical loads (thermoelastic consolidation), are obtained from the theory of mixtures within the framework of the theories advanced by Green and Nagdhi [23] and Crochet and Nagdhi [15]. A general variational principle equivalent to the field equations and boundary conditions is developed using the approach of Sandhu and Pister [50] in constructing variational principles for initial boundary value problems. A finite element formulation in space and time is presented. In the spatial discretization two isoparametric elements of the composite type are presented.

Throughout the development, the usual index notation is employed with the implied summation on repeated indices and the comma denoting spatial derivatives.

\subsection{Balance Equations}

Green and Nadghi [23] considered a mixture of two continua $s_{1}$ and $s_{2}$ occupying a volume $V$ bounded by an area $A$. 
An energy balance equation was postulated in their work for the whole continuum mixture by suitable combinations of the equations deduced for each constituent. The invariance conditions under superimposed rigid body motions were applied to the energy balance equation and the resulting equations for non chemically reacting continuua are as follows:

\subsubsection{Conservation of Mass}

I'he mass conservation cquation for eash ronstituent is

$$
\frac{\partial \rho_{n}}{\partial t}+\left(\rho_{n} v_{k}^{(n)}\right)_{k}=0 \quad \text { for } n=i, 2
$$

where $\rho_{\mathrm{n}}$ and $\mathrm{v}_{\mathrm{k}}^{(\mathrm{n})}$ are mass density and components of velocity vector for $n^{\text {th }}$ constituent respectively. The combined mass density is defined as

$$
\rho=\rho_{1}+\rho_{2}
$$

\subsection{Conservation of Linear Momentum}

The equations for conservation of linear momentum can be expressed as :

$$
\sum_{n=1}^{2} \bar{\sigma}_{k i, k}^{(n)}+\sum_{n=1}^{2} \rho_{n}\left(f_{i}^{(n)}-r_{i}^{\prime}\right)=0
$$

where $\bar{n}_{k 1}^{(n)}, f_{i}^{(n)}$ and $F_{i}^{(n)}$ are the components of the partial 
stress tensor, body force and acceleration vectors for the $\mathrm{n}^{\text {th }}$ constituent, respectively.

\subsubsection{Total Stress Tensor:}

The conservation of angular momentum for the mixture guarantees the symmetry of the total stress, i.e.,

$$
\sum_{n}^{2} \bar{\sigma}_{k i}^{(n)}={ }_{n}^{2} \underline{\Sigma}_{1} \bar{\sigma}_{i k}^{(n)}
$$

Along the boundary, we have

$$
\left.\sum_{n=1}^{2} \bar{\sigma}_{k i}^{(n)}\right) n_{k}=\sum_{n=1}^{2} t_{i}^{(n)}
$$

where $t_{i}^{(n)}$ are the components of surface traction vectors for the $n^{\text {th }}$ constituent.

\subsubsection{Diffusive Resistance}

The diffusive resistance represents the interaction betwen the two continuua and is defined by

$$
\bar{\pi}_{i}=\frac{1}{2}\left(\bar{\sigma}{ }_{k i}^{(1)}-\bar{\sigma}_{k i}^{(2)}\right)_{k}+\frac{1}{2} \rho_{1}\left(f_{i}^{(1)}-F_{i}^{(1)}\right)-\frac{1}{2} \rho_{2}\left(f_{i}^{(2)}-F_{i}^{(2)}\right)
$$

which can be reduced to, in view of $\mathrm{Eq}$ (2.3),

$$
\bar{\pi}_{i}=\rho_{2} \quad\left(F_{i}^{(2)} \underline{f}_{i}^{(2)}\right)-\bar{\sigma}_{k i, k}^{(2)}
$$

and

$$
-\bar{\pi}_{i}=\rho_{l}\left(F_{i}^{(1)}-f_{i}^{(l)}\right)-\bar{\sigma}_{k i, k}^{(1)}
$$




\subsection{Constitutive Equations}

The material behavior needs to be specified in order to apply the balance equations discussed in the preyious section. This behavior is defined by the constitutive equations for stress, entropy, energy, heat flux and flow flux.

Crochet and Naghdi [15] started by admitting fairly general constitutive assumptions prior to placing restrictions by using the clausius-Duhem inequality. Their results are further specialized to the case of infinitesimal theory of fluid flow through a linearly elastic isotropic solid. The continuum is assumed to be initially at rest with constant temperature and zero initial stress. For infinitesimal deformations the solid strain $e_{i j}$ is

$$
e_{i j}=\frac{1}{2}\left(u_{i, j}+u_{j, i}\right)
$$

where $u_{i}$ are the solid displacements.

We let $s_{1}$ be the solid and $s_{2}$ be the fluid. Then at the equilibrium state when the relative velocity and velocity gradients are zero, the constitutive relations are given as

$$
\begin{aligned}
& \bar{\sigma}_{0 i j}^{(I)}=2 \mu_{1} e_{i j}+\lambda_{1} e_{k k} \delta_{i j}-\gamma \cdot \rho_{2} \delta_{i j}-\beta T \delta_{i j}(2.10 a) \\
& \bar{\sigma}_{0 i j}^{(2)}=\left[-K \rho_{2}+\gamma e_{k k}-\zeta T\right] \delta_{i j} \\
& \bar{\pi}_{0 i}=0
\end{aligned}
$$


where the subscript 0 is used to denote the equilibrium values. $T$ is the current temperature and $\mu, \lambda, \gamma, \beta, K, \zeta$ are material constants. Furthermore, the anti-symetric part of partial stresses must vanish at equilibrium, that is,

$$
\bar{\sigma}_{o[i j]}^{(n)}=0 \quad \text { for } n=1,2
$$

The "extra" values of the partial stresses and diffusive resistance are given by

$$
\begin{aligned}
& \bar{\sigma}_{e(i j)}^{(1)}=\sum_{n=1}^{2}\left(\lambda_{3}^{(n)} D_{k k}^{(n)} \delta_{i j}+2 \mu_{3}^{(n)} D_{i j}^{(n)}\right) \\
& \bar{\sigma}_{e(i j)}^{(2)}=\sum_{n=1}^{2}\left(\lambda_{2}^{(n)} D_{k k}^{(n)} \delta_{i j}+2 \mu_{2}^{(n)} D_{i j}^{(n)}\right) \\
& \bar{\sigma}_{e[i j]}^{(1)}=-\bar{\sigma}_{e[i j]}^{(2)}=-c_{1} \Lambda_{i j} \\
& \bar{\pi}_{e i}=c_{3} v_{i}
\end{aligned}
$$

where (.) denotes the symmetric part and [.] denotes the antisymmetric part, $D_{i j}^{(n)}$ are the deformation rates, $n_{i j}$ and $v_{i}$ are relative vorticities and relative velocities, respectively, $c_{1}, c_{3}, \lambda_{3}^{(n)}, \lambda_{2}^{(n)}, \mu_{3}^{(n)}$ and $\mu_{2}^{(n)}$ are material constants. The heat flux is given by

$$
\mathrm{h}_{j}=-\mathrm{k} \mathrm{T}_{, j}+\mathrm{c}_{5} \mathrm{v}_{\mathrm{j}}
$$

where $k$ and $c_{5}$ are material constants. 
For isothermal consolidation, Sandhu [48] assumed that the solid stress is independent of the deformation rates of the solid and fluid, the fluid partial stress is independent of the deformation rate of the solid and vorticity effect is negligible. Thus, combination of Eqs $(2,11)$ and (2.12) yields

$$
\begin{aligned}
& \bar{\sigma}_{i j}^{(1)}=2 \mu_{1} e_{i j}+\lambda_{1} e_{k k} \delta_{i j}-\beta T \delta_{i, j}-\gamma \rho_{2} \delta_{i j}(2.14 a) \\
& \left.\bar{\sigma}_{i j}^{(2)}=1-k \rho_{2}+\gamma e_{k k}-\zeta T\right] \delta_{i j}+2 \mu{ }^{(2)} D_{i j}^{(2)} \\
& \quad+\lambda_{2}^{(2)} D_{k k} \delta_{i j} \\
& \bar{\pi}_{i}=c_{3} v_{i}
\end{aligned}
$$

\subsubsection{Darcy's Law}

Crochet and Nagdhi [15] discussed the possibility of deriving Darcy's law from Eq (2.12). Sandhu [48] used Eq (2.14c) and the definition of diffusive iesistance, Eq (2.7) to obtain Darcy's law for the quasi-static case, namely

$$
c_{3} v_{i}=-\left(\rho_{2} f_{i}^{(2)}+\bar{\sigma}_{k i, k}^{(2)}\right)
$$

By setting $t_{i}^{(1)}=f_{i}^{(2)}=F_{i}$ ald ieplacing $c_{j}$ by $k_{i j}^{-1}$, then

$$
v_{i}=-k_{i j}\left(\bar{\sigma}_{k j, k}^{(2)}+\rho_{2} f_{j}\right)
$$

noting that $v_{i}=v_{i}^{(1)}-v_{i}^{(2)}$ and $v_{i}^{(1)} \ll v_{i}^{(2)}$, then 


$$
v_{i}^{(2)}=k_{i j}\left(\bar{\sigma}_{k j, k}^{(2)}+\rho_{2} f_{j}\right)
$$

Furthermore, Sandhu [48] set $\gamma=0$ in Eq (2.14b) since it contradicts with Darcy's law which postulates that fluid stresses depend only on $\rho_{2}$ and $v_{i}^{(2)}$ and are independent of the solid deformations. Viscosity effects were also ignored by setting $\mu_{2}^{(2)}=\lambda^{(2)}=0$ in Eq $(2.14 \mathrm{~b})$. The resulting equations are

$$
\begin{aligned}
& \bar{\sigma}_{i j}^{(1)}=2 \mu_{1} e_{i j}+\lambda_{1} e_{k k} \delta_{i j}-\beta T \delta_{i j} \\
& \bar{\sigma}_{i j}^{(2)}=\left[-K \rho_{2}-\zeta T\right] \delta_{i j} \\
& \bar{\pi}_{i}=c_{3} v_{i}
\end{aligned}
$$

\subsubsection{Continuity Equation}

The divergence of the relative velocity yields, in view of Eq (2.16),

$$
\dot{e}_{i i}-\dot{\varepsilon}_{i i}=k_{i j}\left(\bar{\sigma}, j+\rho_{2} f_{j}\right), i
$$

where $\dot{e}_{i i}=v_{i, i}^{(1)}, \dot{\varepsilon}_{i i}=v_{i, i}^{(2)}$, and $\bar{\sigma}^{(2)}=\bar{\sigma}_{i i}^{(2)} / 3$.

For an incompressible fluid, $\dot{\rho}_{2}=0$, and by virtue of Eq (2.1), the continuity equation can be expressed as

$$
\dot{e}_{i i}+k_{i j}\left(\ddot{\sigma}(2)+\rho_{2} f_{j}\right)=0
$$




\subsubsection{Fourier's Law}

In the present analysis, the effect of velocity on heat flux is ignored, since the process is quasi-static, by setting $c_{5}=0$ in Eq (2.13). By writing $\mathrm{k}$ as a symmetric tensor of the second order in Eq (2.11) gives

$$
h_{i}=-k_{i j} T_{, j}
$$

\subsubsection{Energy Equation}

Accorting to Crochet and Nagdhi [15], the entropy per unit llids of the mixture can be written as

$$
S=S_{0}+\frac{C}{\tau_{0}} T+\frac{\beta}{\rho_{0}} e_{i i}-\frac{\zeta}{\rho_{0}} \frac{\rho_{2}}{\rho_{20}}
$$

where $\mathrm{S}_{0}$ is the reference value of the entropy, $c, \beta$ and $\zeta$ are material constants and $\tau_{0}$ is the reference temperature. The rate of change of entrupy is given by, [20]

$$
\rho_{0} \frac{\partial S}{\partial t}=-\frac{1}{T} h_{i, i}+\frac{n_{0}}{T} r
$$

where $r$ is the heat supply function. Thus, for an incompressible fluid, substituting from Fig (2.22) into Eq (2.23) yields

$$
\frac{c \rho_{o}}{\tau_{0}} \dot{T}+\beta \dot{e}_{i i}+\frac{1}{T} h_{i, i}-\frac{1}{T} \rho_{o} r=0
$$

Substituting for $h_{i}$ from $\mathrm{Eq}(2.21)$ and after linearization yields 


$$
\rho_{0} C \dot{T}+\beta \tau_{0} \dot{e}_{i i}=k_{i j} T, j i+\rho_{0} r
$$

As mentioned earlier, Crochet and Nagdhi [15] assumed that the solid is isotropic. The mechanical anisotropy can be introduced by a fourth order tensor $E_{i j k l}$ in lieu of $\lambda$ and $\mu$ in Eq.(2.18). Hydraulic and thermal anisotropy can be noted in the two second order tensors $k_{i j}$ and $k_{i j}$ ' respectively. It is also worth mentioning that the three types of anisotropy are mutually independent.

Before closing this section, the notations for solid and fluid partial stresses will be changed to be in line with the commonly used notation in the isothermal theory of consolidation $[48,49]$. The effective stress in the solid will be denoted by $\bar{\sigma}_{i j}$ instead of $\bar{\sigma}_{i j}^{(1)}$ and $\bar{\sigma}_{i j}^{(2)}$ will be replaced by $\pi_{i j}$ to denote the pore pressure. That is,

$$
\begin{array}{ll}
\sigma_{i j}=E_{i j k l} e_{k l} & \begin{array}{l}
\text { is the effective isothermal } \\
\text { stress tensor }
\end{array} \\
\bar{\sigma}_{i j}=\sigma_{i j}-\beta T \delta_{i j} & \text { is the effective stress tensor } \\
\bar{\sigma}_{i j}=\bar{\sigma}_{i j}+\pi \delta_{i j} & \text { is the total stress tensor }
\end{array}
$$

\subsection{Variational Formulation}

\subsubsection{General}

Finite element formulations often rely on variational principles. In this section, a variational principle for the 
problem of thermoelastic consolidation will be developed.

Sandhu and Pister [50] extended Mikhlin's variational problem [34] to the case of coupled problems which involve several dependent variables. The set of variables are treated as a single vector variable and the set of operators are replaced by a single generalized symmetric operator.

Sandhu and Salaam 152J included the non humugentuus boundary rnnditions in the variational formulation by writing the boundary operator in a "consistent" form with the field operator. As an example, consider the boundary value problem expressed by

$$
\mathrm{Au}=\mathrm{f} \text { on } \mathrm{R}
$$

with $\mathrm{Cu}=\mathrm{b}$ on $\mathrm{\partial R}$ $(2.27)$

where $A$ is a self adjoint operator with respect to a symmetric nondegenerate bilinear map, and $c$ is a boundary operator consistent with $A, 1 . e .$, il should satiof $f_{Y}$

$$
\int_{R} \text { UAvaR }=\int_{R}^{\int v A u d R} i \underset{\partial R}{\int}(\mathrm{C} v-v C u) d S
$$

The variational principle equivalent to the problem is given by

$$
J[u]=\underset{R}{\int}(u A u-2 u f) d R+\underset{\partial R}{\int}(u C u-2 u b) d S
$$


The vanishing of the variation of $J[u]$ among all kinematically admissible functions implies Eqs (2.26) and (2.27).

For an initial boundary value problem, Gurtin [24] proposed a bilinear map defined as the spatial integral of the convolution product, to include initial conditions explicitly in the variational principle,

$$
\langle w, v\rangle=\int_{R}^{\int} \int_{0}^{t} w(\underset{\sim}{x}, t) v(\underset{\sim}{x}, t-\tau) d \tau d R
$$

It was shown that the vanishing of the variation of $\mathrm{J}[\mathrm{u}]$ is equivalent to vanishing of its Gateaux differential, defined by

$$
\Delta_{v} J[u]=\left.\frac{d}{d \lambda} J[u+\lambda v]\right|_{\lambda=0}
$$

The above approach will be used in constructing a variational principle for the initial boundary value problem of thermoelastic consolidation. Extended variational principles together with some specializations are also presented.

\subsubsection{The Initial Boundary Value Problem of Thermoelastic Consolidation}

The field equations governing the flow of an incompressible fluid through a linearly elastic homogenous solid subjected to both thermal and mechanical loads were presented in Section 2.3, and will formally be restated here. 
We let $\mathrm{R}$ be an open connected region occupied by the fluid-solid mixture, $\partial \mathrm{R}$ its boundary and $\overline{\mathrm{R}}$, its closure. The domain of definition of all the variables is $\bar{R} \times[0, \infty]$ is the non-negative interval of time. The field equations are:

(i) stress-strain and strain displacement relations for a linear elastic solid matrix

$$
\begin{aligned}
& o_{i j}=E_{i j k l} e_{k l} \\
& e_{i j}=\frac{1}{2}\left(u_{i, j}+u_{j ; i}\right)
\end{aligned}
$$

(ii) equilibrium equations for the solid-fluid mixture

$$
\left(\sigma_{i j}+\pi \delta_{i j}-\beta T \delta_{i j}\right)_{, j}+\rho f_{i}=0
$$

(iii) Darcy's law for irrotational fluid flow

$$
q_{i}=k_{i j} \theta_{j}
$$

where $\theta_{j}$ is given by

$$
\theta_{j}=\pi, j+\rho_{2} f_{j}
$$

(1v) the curlinuity equation for a non-rhemically reacting continuum with the solid skeleton fully saturated by an incompressible fluid

$$
q_{i, i}=-\dot{e}_{i i}
$$

(v) Fourier's law of heat condition

$$
h_{i}=-k_{i j} \phi_{j}
$$


where $\phi_{j}$ is given by

$$
\phi_{j}=T, j
$$

(vi) an energy equation for the solid-fluid mixture with convection ignored

$$
h_{i, i}+\rho c_{v} \dot{T}+\beta \tau_{0} \dot{e}_{i i}=\rho_{0} r
$$

The boundary conditions associated with the problem are (a) displacement boundary conditions

$$
u_{i}(\underset{\sim}{x}, t)=\hat{u}_{i}(\underset{x}{x}, t) \quad \text { on } s_{1} x[0, \infty)
$$

where $S_{1} \subset \partial R$

(b) traction boundary conditions

$$
t_{i}(\underset{\sim}{x}, t)=\hat{t}_{i}(\underset{\sim}{x}, t) \text { on } s_{2} x[0, \infty)
$$

where $t_{i}(\underset{\sim}{x}, t)=\left(\sigma_{i j}+\pi \delta_{i j}-\beta T \delta_{i j}\right) n_{j}$

where $n_{j}$ is the outward unit normal to $s_{2}$ and $s_{2} \subset \partial R$ such that

$$
\mathrm{s}_{1} \cap \mathrm{s}_{2}=\phi \quad \text { and } \overline{\mathrm{s}} \cup \overline{\mathrm{s}}_{2}=\partial \overline{\mathrm{R}}
$$

(c) pore pressure boundary conditions

$$
\pi(\underset{\sim}{x}, t)=\hat{\pi}(\underset{x}{x}, t) \quad \text { on } s_{3} x[0, \infty)
$$

where $\mathrm{s}_{3} \subsetneq \partial \mathrm{R}$

(d) Fluid flow boundary conditions

$$
Q(\underset{\sim}{x}, t)=\hat{Q}(\underset{\sim}{x}, t) \quad \text { on } S_{4} x(0, \infty)
$$


where

$$
Q=q_{i} n_{i}
$$

where $\mathrm{n}_{i}$ is the outward unit normal to $\mathrm{s}_{4}$ and $\mathrm{s}_{4} \subset \mathrm{R}$ such that

$$
\mathrm{S}_{3} \cap \mathrm{S}_{4}=\phi \text { and } \overline{\mathrm{S}}_{3} \cup \overline{\mathrm{S}}_{4}=\partial \overline{\mathrm{R}}
$$

(e) temperature boundary conditions

$$
T(\underset{\sim}{x}, L)=\hat{T}(\underset{x}{x}, L) \quad \text { un } S_{5} \times[0, \infty)
$$

where $\mathrm{S}_{5} \subset \partial \mathrm{R}$

(f) heat flux boundary conditions

$$
H(x, t)=\hat{H}(x, t) \quad \text { on } S_{6} x[0, \infty)
$$

where $\mathrm{H}=\mathrm{h}_{\mathrm{i}} \mathrm{n}_{\mathrm{i}}$

where $n_{i}$ is the outward unit normal to $s_{6}$ and $s_{6} \subset \partial R$ such that

$$
\mathrm{s}_{5} \cap \mathrm{s}_{6}=\phi \text { and } \overline{\mathrm{s}}_{5} \cup \overline{\mathrm{s}}_{6}=\partial \overline{\mathrm{R}}
$$

The initial conditions for the temperature and displacement are

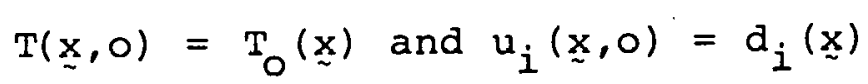

\subsubsection{Alternative Forms for Continuity and Energy Equations}

Following Gurtin [24], an integral form for both of the continuity and energy equations is required to include the initial conditions explicitly in the variational principle. This can be done by Iaplace transformation followed by an inversion. For the continuity equation, considering Laplace transformation of Eq (2.20), we have 


$$
d_{i, i}-s \bar{u}_{i, i}=\bar{q}_{i, i}
$$

where a superposed bar denotes the transformed function and $\mathbf{s}$ is the transformation parameter. Dividing Eq $(2.51)$ by $s$ and inverting back after use of the convolution theorem:

$$
d_{i, i}-u_{i, i}=g^{\prime} * q_{i, i}
$$

where

$$
g^{\prime}(t)=1 \quad v t \in(0, \infty)
$$

and * denotes the convolution integral. The equivalence of Eq 2.52 with Eq 2.20 can be shown as follows. Assuming Eq (2.20) to hold,

$$
\begin{aligned}
g^{\prime} * q_{i, i}=l * q_{i, i}=\int_{0}^{t} q_{i, i} d \tau & =-\int_{0}^{t} u_{i, i} d \tau \\
& =-u_{i, i}+d_{i, i}
\end{aligned}
$$

conversely, assuminy (2.52) to hold. The right hand side of Eq (2.52) is

$$
g^{\prime} * q_{i, i}=\int_{0}^{t} q_{i, i} d \tau
$$

while the left hand side is 


$$
d_{i, i}-u_{i, i}=u_{i, i}(0)-u_{i, i}(t)=-\int_{0}^{t} u_{i, i}(\tau) d \tau
$$

Hence according to $\mathrm{Eq}(2.52)$

$$
\int_{0}^{t}\left(u_{i, i}+u_{i, i}\right) d \tau=0
$$

thereby yielding Eq (2.20).

For the energy equation, we consider Laplace transformation for $\mathrm{Eq}(2.25)$, namely

$$
\bar{h}_{i, i}+\rho c_{v}\left(s \bar{T}-T_{o}\right)+\beta \tau_{o}\left(s \bar{u}_{i, i}-d_{i, i}\right)=\rho \bar{r}
$$

We divide Eq (2.58) by $s$ and invert back using the convolution theorem to obtain

$$
y^{\prime} * h_{i, i}+\rho c_{v}\left(T-T_{0}\right)+\beta \tau_{0}\left(u_{i, i}-d_{i, i}\right)=g^{\prime} * \rho r
$$

The equivalence of Eqs (2.59) and (2.25) can be proved in a manner similar to that demonstrated for the continuity equation.

\subsubsection{Variational Principle}

In order to transform the initial boundary value problem of thermoelastic consolidation into an equivalent variational principle, an admissible state, denoted by $w=\{\underset{\sim}{u}, \underset{\sim}{e}, \sigma, \pi, \underset{\sim}{q}, \underset{\sim}{\theta}, T, \underset{\sim}{h}, \underset{\sim}{\phi}\}$ is defined on $\overline{\mathrm{R}} \times(0, \infty)$ and $t, Q, H$ are 
defined on $\partial R x[0, \infty)$. The set of all $w$ is a linear vector space. The solution to the problem is defined as an admissible state $w_{0}$, which satisfies the field equations as well as initial and boundary conditions. $t, Q$ and $H$ are not considered separately in the admissible state and are assumed in the form $t_{i}=\left(\sigma_{i j}+\pi \delta_{i j}-\beta T \delta_{i j}\right) n_{j}, Q=q_{i} n_{i}$ and $\mathrm{H}=\mathrm{h}_{\mathrm{i}} \mathrm{n}_{\mathrm{i}}$.

The bilinear mapping used in constructing the variational principle is the one proposed by Gurtin [24], Eq (2.30). The field equations are presented by an operator $A$ of 9x9 matrix. The boundary operators are written in a 'consistent' form with the field equations in the sense of Sandhu and Salaam [52]. The field Eqs (2.32) through (2.40) along with Eqs (2.52) and (2.59) can be rewritten as

$$
A w=v \quad \text { on } \bar{R} \times[0, \infty)
$$

where $A, w$ and $v$ are as follows.

$$
\begin{aligned}
& w^{T}=\left\{u_{i}, e_{k l}, \sigma_{i j}, q_{i}, \theta_{j}, \frac{1}{\tau_{o}} \phi_{j}, h_{i}, T\right\} \\
& v^{T}=\left\{\rho f_{k}, 0,0, g^{\prime} \star^{\rho_{2}} f_{j}, 0,0,0,0,0,\right\} \\
& A_{i j}=0 \text { except the following entries: } \\
& A_{13}=-\frac{1}{2}\left(\delta_{i k} \frac{\partial}{\partial j}+\delta_{j k} \frac{\partial}{\partial}\right), A_{16}=-\frac{\partial}{\partial i} \delta_{k i}
\end{aligned}
$$




$$
\begin{aligned}
& A_{19}=\frac{\partial}{\partial i} B \delta_{k i} \quad, \quad A_{22}=E_{i j k l} \\
& A_{23}=-1 \quad, A_{31}=\frac{1}{2}\left(\delta_{k i} \frac{\partial}{\partial 1}+\delta_{1 i} \frac{\partial}{\partial_{k}}\right) \\
& \mathrm{A}_{32}=-1 \quad, \mathrm{~A}_{45}=\mathrm{g}^{\prime *} \\
& A_{46}=g^{\prime *} \frac{\partial}{\partial_{j}} \quad, A_{54}=g^{\prime *} \\
& A_{55}=-K_{i j} * \quad, A_{61}=\frac{\partial}{\partial k} \delta_{i k} \\
& A_{64}=g^{\prime}+\frac{\partial}{\partial_{i}} \quad, A_{77}=-\tau_{0} k_{i j} * \\
& A_{78}=-g^{\prime *} \quad, A_{87}=g^{\prime *} \\
& A_{89}=\frac{1}{\tau_{0}} g^{\prime *} \frac{\partial}{\partial_{j}} \quad, \quad A_{91}=-\frac{\partial}{\partial_{k}} B \delta_{i k} \\
& A_{98}=-\frac{I}{\tau_{0}} g^{\prime} * \frac{\partial}{\partial_{i}} \quad, A_{99}=-\frac{\rho C_{V}}{\tau_{0}}
\end{aligned}
$$

Similarly, the boundary conditions can be written as

$$
\mathrm{Cw}=\mathrm{v}
$$

where

$$
\begin{gathered}
w^{T}=\left\{u_{i}, \bar{\sigma}_{i j}, Q, \pi, H, T\right\} \\
v^{T}=\left\{\hat{t}_{i},-n_{j} \hat{u}_{i}, g^{\prime} * \hat{\pi}_{,}-g^{\prime} * \hat{Q}, g^{\prime} * \hat{T} / \tau_{0},-g^{\prime} * \hat{H} / \tau_{0} \cdot\right\}
\end{gathered}
$$




$$
\begin{array}{ll}
c_{i j}=0 \text { except the following entries: } \\
c_{12}=n_{j} & , c_{21}=-n_{j} \\
c_{34}=g^{\prime *} & , c_{43}=-g^{\prime *} \\
c_{56}=\frac{1}{\tau_{0}} g^{\prime *} & , c_{65}=-\frac{1}{\tau_{0}} g^{\prime *}
\end{array}
$$

where $\overline{\bar{\sigma}}_{i j}$ is the total stress tensor, i.e.,

$\overline{\bar{\sigma}}_{i j}=\sigma_{i j}+\pi \delta_{i j}-\beta T \delta_{i j}$.

In this coupled problem, the uncoupled operators are symmetric, while the coupling operators constitute a pair of adjoint operators with respect to the bilinear map. Green's theorem can be employed to represent the boundary terms associated with the coupling operators (non-zero off diagonal terms of $A$ ), i.e.,

$$
\begin{aligned}
& \int_{R} u_{i}{ }^{\star} \sigma_{i j, j} d R=-\int_{R} u_{i, j}{ }^{*_{\sigma}}{ }_{i j} d R+\int_{\partial R} u_{i}{ }^{*_{\sigma}}{ }_{i j} n_{j} d S \\
& \int_{R} \pi{ }^{*} u_{i, i} d R=-\int_{R} \pi, i{ }^{*} u_{i} d R+\int_{\partial R} \pi * u_{i} n_{i} d S \\
& \int_{R}^{\int \pi *} g^{*}{ }^{*} q_{i, i} d R=\underset{R}{-\int \pi},{ }^{*} g^{\prime}{ }^{*} q_{i} d R+\int_{\partial R} \pi * g^{\prime}{ }^{*} q_{i} n_{i} d S \\
& \int_{R} T * B u_{i, i} d R=-\int_{R} \beta T, i{ }^{*} u_{i} d R+\int_{\partial R} \beta T * u_{i} n_{i d S}
\end{aligned}
$$




$$
\int_{R} T^{*} g^{\prime} \star^{h^{h}} \frac{i}{\tau_{0}} d R=-\int_{R} T,{ }^{*} g^{\prime *} \frac{h_{i}}{\tau_{0}} d R+\underset{\partial R}{\int} T^{*} g^{\prime}{ }^{*} \frac{h_{i} h_{i}}{\tau_{0}} d S
$$

Starting from the initial undeformed state, i.e., $\mathrm{d}_{i}=0$, and $\mathrm{T}_{0}=0$, and following the approach presented by Eq (2.29), the following variational principle is presented.

\section{THEOREM I}

Let $w$ be the oct of all admiojible states. Lel

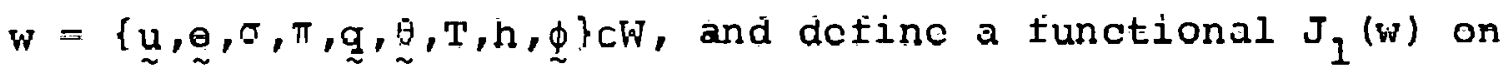
W by

$$
\begin{aligned}
& J_{I}(w)=\int_{R} u_{i}{ }^{*}-\left[\left(\sigma_{i j}+\pi \delta_{i j}-\beta T \delta_{i j}\right)_{, j}+2 \rho f_{i}\right] d R \\
& +\int_{R} e_{i j}{ }^{*}\left[E_{i j k 1} e_{k l}-\sigma_{i j}\right] d R+\int_{R} \sigma_{i j}{ }^{*}\left[u(i, j)-e_{i j}\right] d R
\end{aligned}
$$

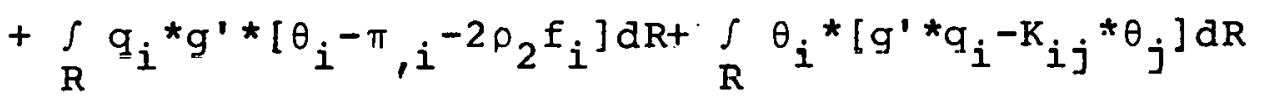

$$
\begin{aligned}
& +\int_{R} \pi *\left[u_{i, i}+g^{\prime} *_{q_{i, i}}\right] d R-\int_{i} \frac{\phi_{i}}{\tau_{0}} *\left[k_{i j}{ }^{*} \phi_{j}+g^{\prime} *_{h_{i}}\right] d R \\
& +\int_{R} h_{i}{ }^{*} g^{\prime *}\left(-\frac{\phi_{i}}{\tau_{0}}+\frac{T, i}{\tau_{0}}\right) d R-\int_{R} T *\left[B u_{i, i}+\frac{l}{\tau_{0}} g^{\prime} * h_{i, i}+\frac{\rho C_{v} T}{\tau_{0}}\right] d \bar{R} \\
& -\int_{s_{1}}\left(\sigma_{i j}+\pi \delta_{i, j}-\beta T \delta_{i, j}\right) *\left(n_{j} u_{i}-2 n_{j} \hat{u}_{i}\right) d s+ \\
& +\int_{S_{2}} u_{i} *\left(t_{i}-2 \hat{t}_{i}\right) d S+\int_{S_{3}} Q^{*} g^{* *}(\pi-2 \hat{\pi}) d S-
\end{aligned}
$$




$$
-\int_{S_{4}} \pi^{*} g^{\prime *}(Q-2 \hat{Q}) d S-\int_{S_{5}} H * \frac{g^{\prime}}{\tau_{0}} *(T-2 \hat{T}) d S+\int_{S_{6}} T * \frac{g^{\prime}}{\tau_{0}^{\prime}} *(H-2 \hat{H}) d S
$$

where ${ }_{\sim}, Q$, and $H$ are defined by Eqs.(2.43),(2.46) and (2.49), respectively.

It can be shown that the Gateaux differential of $J_{1}(w)$ along an arbitrary path $\bar{w} \in W$ vanishes, if and only if the field equations together with the boundary conditions are satisfied. The proof of this theorem is given in Appendix F.

\subsubsection{Extended Variational Principles}

As mentioned earlier, Green's theorem can be used to represent the relations between the coupling operators. Thus, one or more of the adjoint operators can be eliminated from the basic variational principle resulting in many alternative forms. This will induce an extension in the domain of definition of $J_{l}(w)$. For example, the term $\sigma_{i j, j}$ can be eliminated from $J_{l}(w)$ by using $\mathrm{Eq} \cdot(2.68)$.

Then, by eliminating $\sigma_{i j, j} ; \pi, i, B T, i, g^{\prime+} q_{i, i}$ and $\frac{l}{\tau_{0}} g^{\prime *} h_{i, i}$ from $J_{l}(w)$, the following variational principle is obtained:

$$
\begin{aligned}
& J_{2}(w)=-2 \int_{R} u_{i}{ }^{\star} \rho f_{i} d R+\int_{R} e_{i j}{ }^{\star} E_{i j k l} e_{k l} d R+2 \int_{R} \sigma_{i j}{ }^{*}\left[u_{i, j}-e_{i j}\right] d R \\
& +\int_{R} q_{i}{ }^{*} g^{\prime *}\left[\theta_{i}-2\left(\pi, i^{+\rho} 2_{i} f_{i R}+\int_{R} \theta_{i}{ }^{*}\left[g^{\prime} * q_{i}-K_{i j}{ }^{\theta}\right] d R\right.\right. \\
& +2 \int_{R} \pi^{*} u_{i, i} d R+\int_{R} \frac{\phi_{i}}{\tau_{0}} *-\left[g^{\prime}{ }^{*} h_{i}+k_{i j}{ }^{*} \phi_{j}\right] d R
\end{aligned}
$$




$$
\begin{aligned}
& +\int_{R} T *\left[-2 \beta u_{i, i}+\frac{\rho C_{v}}{\tau_{0}}\right] d R+\int_{R} h_{i}{ }^{*} g^{\prime} *\left[-\frac{\phi_{i}}{\tau_{0}}+\frac{2 T, i}{\tau_{0}}\right] d R \\
& -2 S_{S_{2}} u_{i} * \hat{t}_{i} d s-2 S_{S_{1}}\left(\sigma_{i j}{ }^{*} \pi \delta_{i j}-\beta T \delta_{i j}\right) *\left(n_{j} u_{i}-n_{j} \hat{u}_{i}\right) d s
\end{aligned}
$$

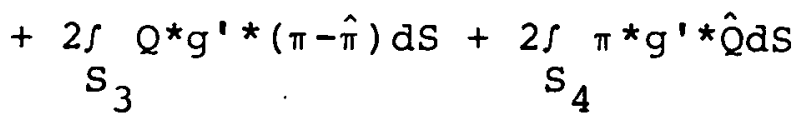

$$
\begin{aligned}
& -2 \int_{S_{5}} H * \frac{g^{\prime}}{\tau_{0}} *(T-\hat{T}) d S-2 S_{S_{6}} T * \frac{g^{\prime}}{\tau_{0}} * \hat{H} d S
\end{aligned}
$$

\subsubsection{Specializations}

One of the interesting specializations is to require the aduissible slale in $J_{2}(w)$ lo ideidlieally, sälisfy,

$$
\begin{aligned}
& e_{i j}=\frac{1}{2}\left(u_{i, j}+u_{j, i}\right) \\
& \sigma_{i j}=E_{i j k l} e_{k l} \\
& \theta_{i}=\pi, i+\rho_{2} f_{i} \\
& q_{i}=k_{i j}{ }_{j} \\
& \text { on } \bar{R} \times[0, \infty) \\
& \phi_{i}=' I ', i \\
& h_{1}=-k_{1 f} \phi_{f} \\
& u_{i}=\hat{u}_{i} \\
& \text { on } S_{1} \times(0, \infty) \\
& \pi=\hat{\pi} \\
& \text { on } s_{3} \times[0, \infty) \\
& \mathrm{T}=\hat{\mathrm{T}} \\
& \text { in } s_{5} \times[0, \infty)
\end{aligned}
$$

i.e., the admissible state is completely defined by $\{\underline{u}, \pi, T\}$. The resulting variational functional is 


$$
\begin{aligned}
& J_{3}(\underset{\sim}{u}, \pi, T)=-2 \int_{R} u_{i}{ }^{*} \rho f_{i}+\int_{R} e_{i j}{ }^{*} \sigma_{i j} d R-\int_{R} q_{i}{ }^{*} g^{\prime} \star_{\theta} d R \\
& +\int_{R} h_{i}{ }^{*} g^{\prime *} \frac{\phi_{i}}{\tau_{0}} d R+\underset{R}{2} \pi^{*} u_{i, i} d R-\underset{R}{2 \int} \beta T * u_{i, i} d R \\
& -\int_{R} T * \frac{\rho C_{V}}{\tau_{0}} T d R-2 S_{S_{2}} u_{i} * \hat{t}_{i} d S+2 S_{S_{4}} \pi^{*} g^{\prime} * \hat{Q} d S \\
& -2 \int_{S_{6}} T^{*} g^{\prime} * \frac{\hat{H}}{\tau_{0}} d S
\end{aligned}
$$

It is worth noting that the variational principle above is a generalization of the one proposed by Sandhu and Wilson [49] for isothermal consolidation, and the one proposed by Nickell and Sackman [39] for linear coupled thermoelasticity. This functional forms the basis of the finite element discretization in the next section.

\subsection{Finite Element Formulations}

The element displacement, pore pressure and temperature are defined in terms of a set of generalized coordinates. Vanishing of the variation of the spatial discretization results in a set of the first order linear differential equations in time. Further discretization in the time domaln yields a set of linear algebraic equations.

\subsubsection{Spatial Discretization}

For a typical element $m$, the displacement, pressure and temperature are expressed in terms of its nodal values as,

$$
u_{i}^{m}(x, t)=\left[N_{u}^{m}(x)\right]\{u(t)\}
$$




$$
\begin{aligned}
& \pi^{m}(x, t)=\left\{N_{\pi}^{m}(x)\right\}\{\pi(t)\} \\
& T^{m}(x, t)=\left\{N_{T}^{m}(x)\right\}\{T(t)\}
\end{aligned}
$$

The strains, volumetric strains, pressure and temperature gradients, are obtained from the above relations as

$$
\begin{aligned}
& e^{m}(\underset{\sim}{x}, t)=\left[N^{m}(\underset{\sim}{x})\right]\{u(t)\} \\
& \Delta^{m}(\underset{x}{x}, t)=\left[N_{\Delta}{ }^{m}(\underset{\sim}{x})\right]\{u(t)\} \\
& \nabla \pi^{m}(\underset{\sim}{x}, t)=\left[N_{q}{ }^{m}(\underset{\sim}{x})\right]\{\pi(t)\} \\
& \nabla T^{\prime \prime \prime}(\underset{\sim}{x}, t)=\left[N_{h}{ }^{1 i l}(\underset{\sim}{x})\right]\{T(t)\}
\end{aligned}
$$

By considering $M$ elements, and substituting from Eqs. (2.77) through (2.83) into Eq.(2.76) yields a discrete form of the functional,

$$
\begin{aligned}
& J_{3}^{h}(u, \pi, T)=\sum_{m-1}^{M} \int_{m}\left(-2\{u\}^{T}\left[N_{u}{ }^{m}\right]^{T} *\left\{\rho f^{m_{3}}\right\}\right. \\
& +\{u\}^{T}\left[N_{e}\right]^{T}\left[E^{m}\right] *\left[N_{Q} m^{m}\right\}\{u\} \\
& -\left(\{\pi\}^{T}\left[N_{q}{ }^{m}\right]^{T}+\left\{\rho_{2} f^{m}\right\}\right) * g^{\prime} *\left[K^{m}\right]\left(\left[N_{q}{ }^{m}\right]\{\pi\}+\left\{\rho_{2} f^{m}\right\}\right) \\
& +\{T\}^{T}\left[N_{h}{ }^{m}\right]^{T} *-g \cdot * \frac{l}{\tau_{0}}\left[k^{m}\right]\left[N_{h}{ }^{m}\right]\{T\} \\
& +2\{\pi\}^{\mathrm{T}}\left\{\mathrm { N } _ { \pi } \mathrm { m } ^ { \prime } \mathrm { l } ^ { \mathrm { l } } * \left\{\mathrm{N}_{\Delta} \mathrm{m}^{\mathrm{m}}\{\mathrm{u}\}\right.\right. \\
& -2 \beta m_{\{T\}} T_{\left\{N_{T}\right.} m_{\}} *\left\{N_{\Delta} m\right\}\{u\} \\
& -\frac{\rho C_{v}}{\tau_{0}}\{T\} T_{\left\{N_{T}\right.} m_{T} T_{*}\left\{N_{T} m_{j}\{T\}\right) d R_{m} \\
& \left.-2 \int \operatorname{su}_{2}\right\}^{T}\left[N_{u}{ }^{m}\right]^{T} *\left\{\iota^{m}\right\} d S
\end{aligned}
$$




$$
\begin{aligned}
& +\int_{S_{4}}\{\pi\}^{T} \mathrm{~T}_{\mathrm{m}}\left\{\mathrm{N}_{\pi}\right\}^{\mathrm{T} \star^{\prime} \mathrm{g}^{\prime}{ }^{*} \hat{\mathrm{Q}}_{\mathrm{m}} \mathrm{dS}} \\
& -2 S_{6}\{T\}^{T}{ }^{T}\left\{N_{T}\right\}^{T}{ }^{T} g^{\prime *} \hat{H}_{\tau_{0}} d S
\end{aligned}
$$

We define

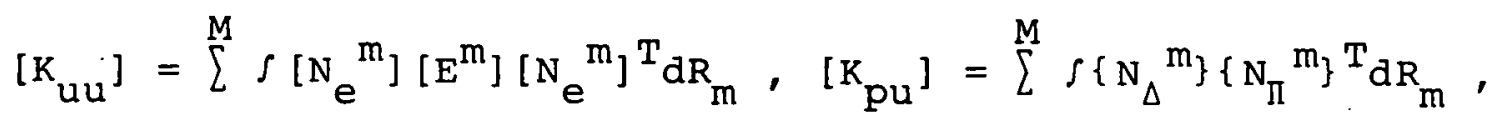

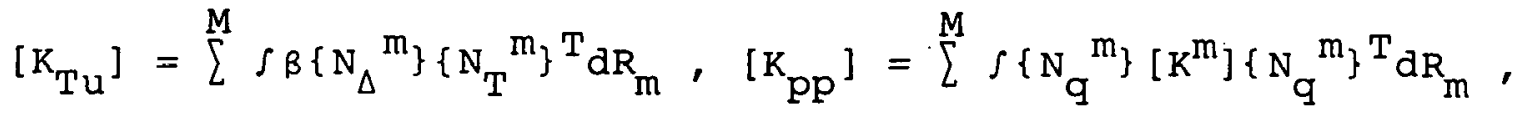

$$
\begin{aligned}
& {\left[\mathrm{K}_{\mathrm{TT}}\right]=\sum^{\mathrm{M}} \int\left\{\mathrm{N}_{\mathrm{h}}{ }^{\mathrm{m}}\right\}\left[\mathrm{k}^{\mathrm{m} / \tau_{0}}\right]\left\{\mathrm{N}_{\mathrm{h}}{ }^{\mathrm{m}}{ }^{\mathrm{T}} \mathrm{dR_{ \textrm {m } }}\right. \text {, }}
\end{aligned}
$$

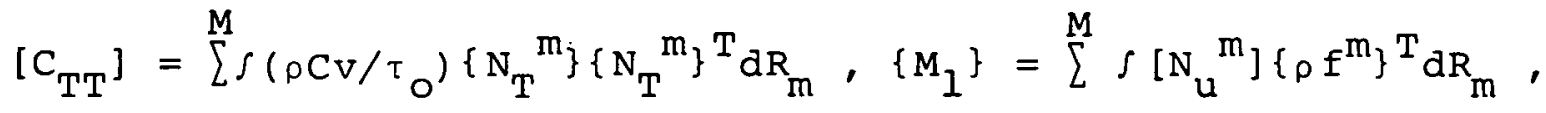

$$
\begin{aligned}
& \left\{M_{2}\right\}=\sum \int\left[N_{q}{ }^{m}\right]\left[K^{m}\right]\left\{\rho_{2} f^{m^{m}}\right\} d R_{m},\left\{M_{3}\right\}=\sum^{M} s\left[N_{u}{ }^{m}\right]\left\{\hat{t}_{m}\right\} d s_{2}^{m} \text {, }
\end{aligned}
$$

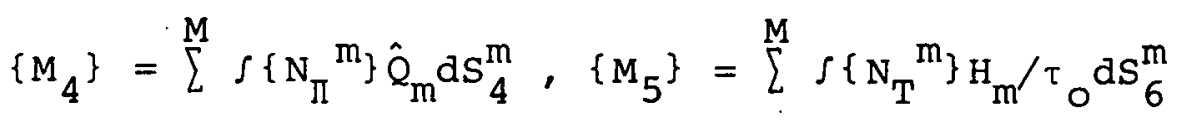

Using the matrices and vectors defined in (2.85), we can write the discrete functional in the matrix form,

$$
\begin{aligned}
& J_{3}^{h}(\underline{u}, \pi, T)=-2\{u\}^{T}\left\{M_{1}\right\}+\{u\}^{T}\left[K_{u u}\right]\{u\}-2 g^{\prime} *\{\pi\} T_{\left\{M_{2}\right\}} \\
& -g^{\prime} *\{\pi\}^{T}\left[K_{p p}\right][\pi]+g^{\prime} *\{T\}^{T}\left[K_{T T}\right][T\} \\
& -\{T\}^{T}\left[C_{T T}\right]\{T\}+2\{u\}^{T}\left[K_{p u}\right]\{\pi\}-2\{u\}^{T}\left[K_{T u}\right]\{T\} \\
& -2\{u\}\}^{T}\left\{M_{3}\right\}+2 g^{\prime *}\{\pi\}^{T}\left\{M_{4}\right\}-2 g^{\prime *}\{T\}^{T}\left\{M_{5}\right\}
\end{aligned}
$$

Applying the variational principle with respect to $\mathfrak{u}_{, \pi}$, and $T$, respectively, yields 


$$
\begin{aligned}
& {\left[K_{u u}\right]\{u\}+\left[K_{p u}\right]\{\Pi\}-\left[K_{T u}\right]\{T\}=\left\{M_{1}\right\}+\left\{M_{3}\right\}} \\
& {\left[K_{p u}\right]^{T}\{u\}-g^{\prime *}\left[K_{p p}\right]\{\pi\}=g^{\prime} *\left\{M_{2}\right\}-g^{\prime} *\left\{M_{4}\right\}} \\
& -\left[K_{T u}\right]^{T}\{u\}-\left[C_{T T}+g^{\prime} * K_{T T}\right]\{T\}=g^{\prime} *\left\{M_{5}\right\}
\end{aligned}
$$

It is worth noting that Eqs.(2.89), (2.90) and (2.91) represent discrete forms of the equilibrium, continuity and energy equations, respectively.

\subsubsection{Temporal Discretization}

Eqs. (2.88) and (2.89) sontain convolution products. A step forward integration scheme will be adopted by using an interpolation function for the displacement, pressure and temperature with the end conditions as the generalized coordinates.

The logarithmic interpolation schemes proposed by Sandhu [48] are utilized herein, which begins by expressing a time dependent function

$$
f(\tau)=f\left(t_{n-1}\right)+\left[f\left(t_{n}\right)-f\left(t_{n-1}\right)\right] \frac{\operatorname{Ln}(\tau+1)}{\operatorname{Ln}(\Delta t+1)}
$$

within a time interval $\tau \in\left[t_{n-1}, t_{n}\right]$. Then

$$
q^{\prime} *_{f}(\tau)=(1-\alpha) \Delta t f\left(t_{n-1}\right)+\alpha \Delta t f\left(t_{n}\right)
$$

where

$\alpha=1+\frac{1}{\Delta t}-\frac{1}{\operatorname{Ln}(\Delta t+1)}$

Note that various conventional time stepping methods can be identified from (2.91) by properly selecting $\alpha$. 
Substituting from Eq.(2.91) into Eqs.(2.88) and (2.89) gives

$$
\left[\begin{array}{ccc}
\mathrm{K}_{\mathrm{uu}} & \mathrm{K}_{\mathrm{pu}} & -\mathrm{K}_{\mathrm{Tu}} \\
\mathrm{K}_{\mathrm{pu}}^{\mathrm{T}} & -\alpha \Delta t \mathrm{~K}_{\mathrm{pp}} & 0 \\
-\mathrm{K}_{\mathrm{Tu}}^{\mathrm{T}} & 0 & -\left(\mathrm{C}_{\mathrm{TT}}+\alpha \Delta \mathrm{K}_{\mathrm{TT}}\right)
\end{array}\right]\left\{\begin{array}{l}
u\left(t_{n}\right) \\
\pi\left(t_{n}\right) \\
T\left(t_{n}\right)
\end{array}\right\}=\left\{\begin{array}{l}
R_{u}\left(t_{n}\right) \\
R_{\pi}\left(t_{n}\right) \\
R_{T}\left(t_{n}\right)
\end{array}\right\}
$$

where

$$
\begin{aligned}
& R_{u}\left(t_{n}\right)=\left\{M_{1}\right\}+\left\{M_{3}\right\} \\
& R_{\pi}\left(t_{m}\right)= {\left[K_{p u}\right]\left\{u\left(t_{n-1}\right)\right\}+(1-\alpha) \Delta t\left[K_{p p}\right]\left\{\pi\left(t_{n-1}\right)\right\}+} \\
& \alpha \Delta t\left\{M_{2}\left(t_{n}\right)\right\}+(1-\alpha) \Delta t\left\{M_{2}\left(t_{n-1}\right)\right\}-\alpha \Delta t\left\{M_{4}\left(t_{n}\right)\right\}- \\
&(1-\alpha) \Delta t\left\{M_{4}\left(t_{n-1}\right)\right\} \\
& R_{T}\left(t_{n}\right)=-\left[K_{T u}\right]\left\{u\left(t_{n-1}\right)\right\}-\left(\left[C_{T T}\right]+(1-\alpha) \Delta t\left[K_{T T}\right]\right)\left\{T\left(t_{n-1}\right)\right\} \\
&+ \alpha \Delta t\left\{M_{5}\left(t_{n}\right)\right\}+(1-\alpha) \Delta t\left\{M_{5}\left(t_{n-1}\right)\right\}
\end{aligned}
$$

\subsubsection{Choice of Finite Elements}

Since the composite elements are preferable in the isothermal consolidation analysis, Sandhu [53], this approach is extended by presenting two isoparametric composite elements for plane strain thermoelastic consolidation. The first element which will be referred to as the 8-4-4 element has displacements assigned at all nodes, while the pressure and temperature are assigned at the corner nodes only, Fig. (2.1). The second element, whịch will be referred to as the 8-8-8, has displacements, pressure and temperature assigned 
at all the nodes, Fig.(2.2). A numerical comparison revealing the performance of the two elements is presented in Chapter IV. 


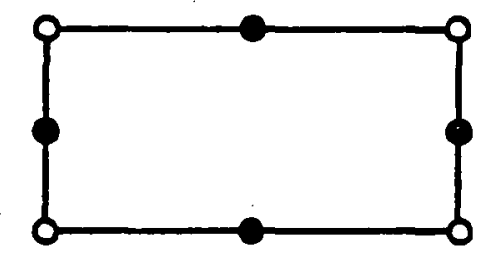

- Nodes with $(u, v, \pi, T)$

- Nodes with $(u, v)$

Figure 2.1. The 8-4-4 Isoparametric Element 


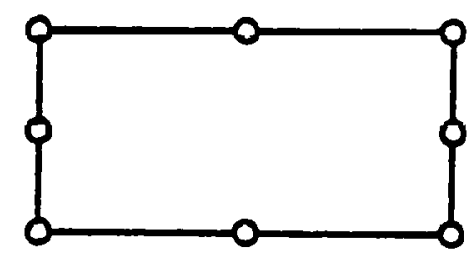

- Nodes with $(u, v, \pi, T)$

Pigure 2.2. The 8-8-8 Isoparametric Element 
CHAPTER III

THERMAL CONSOLIDATION - AN ELASTOPLASTIC ANALYSIS

\subsection{Introduction}

In this chapter, the plastic strain increment due to thermomechanical loading is obtained according to Prager's theory of nonisothermal plastic deformations [42]. The total strain increment is written according to Nadghi [35], and the inverse incremental relation for stress in terms of strain is obtained. The result is specialized to the case of nonisothermal yield surfaces. The incremental form of the field equations is listed and incremental as well as iterative solution schemes are studied. Supplementary information is presented in Appendices A through E. In Appendix $\lambda$, somo classiral yipld criteria and alternative forms are discussed. In Appendix B, the yield surface consisting of the Druckcr-prager line with an expanding elliptic cap is discussed. Appendix $C$ deals with the incremental elastoplastic stress-strain matrix in plane strain and Appendix D discussed the hardening rules and the hardening functions. In Appendix E, numerical methods to obtain the stress increment are discussed. 


\subsection{Plastic Strain Increment}

The effective stress, $\bar{\sigma}_{i j}$, is responsible for all deformations, linear or nonlinear, and failure conditions. For nonisothermal plastic deformations, Prager [42] assumed the yield function in the form

$$
F\left(\bar{\sigma}_{i j}, e_{i j}^{p}, T, k\right)=0
$$

which equivalently can be written as

$$
f\left(\bar{\sigma}_{i j}, e_{i j}^{p}\right)=\sigma_{o}(k, T)
$$

where $\sigma_{0}$ is the uniaxial yield stress of the material, $T$ is the temperature, $e_{i j}^{p}$ is the accumulated plastic strain, and $k$ is a hardening parameter, which can be postulated to be the total plastic work, $\omega_{p}$, i.e.

$$
k=\omega_{p}
$$

where ${ }^{\omega} p$ is given by

$$
\omega_{p}=\int \bar{\sigma}_{i j} d e_{i j}^{p}
$$

Fur the above, the material will be termed as work hardening. Alternatively, $k$ can be related to the measure of the total plastic deformations, termed the equivalent plastic strain, i.e.

$$
k=e_{p}
$$

where

$$
d e_{p}=c\left(d e_{i j}^{p} d e_{i j}^{p}\right)^{1 / 2}
$$

and $c$ is a constant depending on the yield criterion (see Appendix D), and the material in this case will be classified 
as a strain hardening material. The work hardening hypothesis will be assumed throughout this study.

For developing plastic deformations, the consistency condition can be obtained from $\mathrm{Eq} \cdot(3.1)$ as

$$
d F=\frac{\partial F}{\partial \bar{\sigma}_{i j}} d \bar{\sigma}_{i j}+\frac{\partial F}{\partial e_{i j}^{p}} d e_{i j}^{p}+\frac{\partial F}{\partial T} d T+\frac{\partial F}{\partial k} d k=0
$$

We also define

$$
d^{\prime} F=\frac{\partial F}{\partial \bar{\sigma}_{i j}} d \bar{\sigma}_{i j}+\frac{\partial F}{\partial T} d T
$$

Upon unloading from a given plastic state, the stress state moves inwards from the yield surface, i.e. $d F<0$, $d e_{i j}^{p}=0, d k=0$. Thus in this case

$$
F=0, d^{\prime} F<0
$$

If the stress state moves along the yield surface, $d F=0$, then some components of stress increase while others decrease at a constant temperature, keeping the solid in a plastic state without undergoing any plastic deformation. Such a state is termed the neutral change of state. For this case

$F=0, d^{\prime} F=0$

If the stress state moves along the yield surface for all times then $\mathrm{dF}=0$, and plastic loading corresponds to

$$
F=0, \quad d^{\prime} F>0
$$

For the isothermal case prager [42], Boley [7] and Kachanov [26] assumed that $\mathrm{de}_{i j}^{\mathrm{p}}$ is proportional to d'F which characterizes the transition from loading to unloading, i.e.

$$
d e_{i j}^{p}=A_{i j} d^{\prime} F \quad \text { when } \quad F=0, d^{\prime} F>0
$$


The associated flow rule (Appendix A) is assumed to be valid. Consequently, $\mathrm{de}_{i j}^{\mathrm{p}}$ must be directed along the normal to the loading surface. This condition may be fulfilled by setting

$$
A_{i j}=\frac{1}{h} \frac{\partial F}{\partial \bar{\sigma}}
$$

where $h$ is a positive scalar; a consequence of the Drucker stability postulate,

$$
d \sigma_{i j} d e_{i j}^{p}>0 \text { when } F=0 \text { and } d \cdot F>0
$$

By substituting Eq.(3.13) into Eq.(3.12), the plastic strain increment can be given by

$$
d e_{i j}^{p}=\frac{1}{h} d^{\prime} F \frac{\partial F}{\partial \bar{\sigma}_{i j}} \text { when } F=0
$$

The scalar $\mathrm{h}$ in Eq.(3.15) is known as the hardening function which expresses the amount of hardening and can be obtained by substituting Eq.(3.15) into the consistency condition, Eq.(3.7), to give

$$
h=-\left(\frac{\partial F}{\partial e_{1 j}^{p}}+\frac{\partial F}{\partial k} \frac{\partial k}{\partial e_{i j}^{p}}\right) \frac{\partial F}{\partial \bar{\sigma}} i j j
$$

By using the work hardening hypothesis, Eq. (3.4),

F.q. $(3.6)$ gives

$$
h=-\left(\frac{\partial F}{\partial e_{i j}^{P}}+\frac{\partial F}{\partial k} \bar{\sigma}_{i j}\right) \frac{\partial F}{\partial \bar{\sigma}_{i j}}
$$

Calculations of $\mathrm{h}$ for isotropic and mixed hardening are given in Appendices $B$ and $D$. By letting

$$
d \lambda=\frac{1}{h} d \cdot F
$$


Eq. (3.15) becomes

$$
d e_{i j}^{p}=d \lambda \frac{\partial F}{\partial \bar{\sigma}_{i j}}
$$

\section{3 Stress Strain Relation}

The total strain increments are assumed to be the sum of the elastic and plastic strain increments.

$$
d e_{i j}=d e_{i j}^{e}+d e_{i j}^{p}
$$

According to Nagdhi [35], the elastic strain increment can be expressed as

$$
d e_{i j}^{e}=D_{i j k I}^{-1} d \bar{\sigma}_{k I}+\bar{\alpha}_{i j} d T
$$

where $\bar{\alpha}$ is the coefficient of linear thermal expansion, $D_{i j k l}$ is the elasticity tensor. Substituting from Eqs. (3.15) and (3.21) into $\mathrm{Eq} \cdot(3.20)$ gives

$$
d e_{i j}=D_{i j k l}^{-1} d \bar{\sigma}_{k l}+\bar{\alpha} \delta_{i j} d T+\frac{1}{\hbar} d \cdot F_{\frac{\partial F}{i j}}
$$

Sulviny fus the incremental effectlve stress,

$$
d \bar{\sigma}_{k l}=D_{k l i j} d e_{i j}-\bar{\alpha} D_{k l i j}{ }_{i j} d T-\frac{1}{h} D_{k l i j} \frac{\partial F}{\partial \bar{\sigma}_{i j}} d^{\prime} F \text { (3.23) }
$$

which can be used in the consistency condition, Eq. (3.8) to obtain

$$
d^{\prime} F=\frac{\frac{\partial F}{\partial \bar{\sigma}_{k l}} D_{k l i j}\left[d e_{i j}-\bar{\alpha} \delta_{i j} d T\right]+\frac{\partial F}{\partial T} d T}{1+\frac{1}{h} \frac{\partial F}{\partial \bar{\sigma}_{m n}} D_{m n p q} \frac{\partial F}{\partial \bar{\sigma}} p q}
$$

and insertion of Eq.(3.24) into Eq.(3.23) yields 


$$
d \bar{\sigma}_{k l}=D_{k l i j}^{e p}\left[d e_{i j}-\bar{\alpha} \delta_{i j} d T\right]-D_{k I}^{T} d T
$$

where

$$
\begin{aligned}
D_{k l i j}^{e p} & =D_{k l i j}-D_{k l i j}^{p} \\
D_{k l i j}^{p} & =\frac{D_{k l u v} \frac{\partial F}{\partial \tilde{\sigma}_{u v}} \frac{\partial F}{\partial \bar{\sigma}_{s t}} D_{s t i j}}{h+\frac{\partial F}{\partial \bar{\sigma}_{m n}} D_{m n p q} \frac{\partial F}{\partial \bar{\sigma}_{p q}}}
\end{aligned}
$$

and

$$
D_{k l}^{\prime \prime}=\frac{D_{k l u v} \frac{\partial F}{\partial \bar{v}} u v}{\partial T} \frac{\partial F}{\partial+\frac{\partial F}{\partial \bar{\sigma}_{m n}} D_{m n p q} \frac{\partial F}{\partial \bar{\sigma}_{p q}}}
$$

It should be noted that $D_{k l}^{T}$ reflects the dependency of the yield surface on temperature. Unfortunately, however, a yield function that depends explicitly on the temperature is not available in the literature. It is recommended for future research to perform experimental. work to derive the relation between the yield stress and temperature. Thus, although the derived stress-strain relation is written in a general form it is specialized to the case of isothermal yield functions in the sequel. The yield criteria listed in Appendices $A$ and $B$ will be used in the analysis. This assumption had been used in metal thermoplastic analysis Ref.[27]. For an isothermal yield criterion, the last term in $\mathrm{Eq} \cdot(3.25)$ is neglected to yield

$$
d \bar{\sigma}_{k l}=D_{k l i j}^{e p} d e_{i j}-\beta_{k l}^{e p} d T
$$


where

$$
\beta_{k l}^{e p}=\beta_{k l}-\beta_{k l}^{p}
$$

in which

$$
B_{k l}=\bar{\alpha} \delta_{i j}
$$

and

$$
{ }_{\beta}^{\mathrm{P} l}=\bar{\alpha} \delta_{i j} \mathrm{D}_{\mathrm{k} l i j}^{\mathrm{P}}
$$

For an isotropic material,

$$
D_{k l i j}=2 \mu \delta_{i k} \delta_{l j}+\lambda \delta_{i j} \delta_{k l}
$$

Hence

$$
\beta_{k l}=\beta \delta_{k l}
$$

and

$$
\beta{ }_{\mathrm{kl}}^{\mathrm{p}}=\frac{\beta}{\gamma} \frac{\partial}{\partial \bar{\sigma}_{\mathrm{SS}}}\left(2 \mu \frac{\partial F}{\partial \bar{\sigma}_{\mathrm{kl}}}+\lambda \frac{\partial F}{\partial \bar{\sigma}_{\mathrm{uu}}}\right) .
$$

where

$$
\beta=\bar{\alpha}(2+3 \lambda)
$$

and

$$
\gamma=h+\frac{\partial F}{\partial \bar{\sigma}_{m n}} D_{m n p q} \frac{\partial F}{\partial \bar{\sigma}_{p q}}
$$

It should be noted that Eq. (3.35) implies that $\beta \frac{\mathrm{p}}{\mathrm{kl}}$ are zero for the case where in yield surfaces are independent on the first stress invariant, $I_{i}$, e.g., Tresca and Von Mises. This, however, is not the case for Drucker -Prager, Mohr-Coulomb and the cap yield criteria frequently used in geotechnical applications. 
Eq. (3.29) can be put into a more suitable computaticnal form, viz.,

$$
\begin{aligned}
d \bar{\sigma}_{k l} & =D_{k l i j} d e_{i j}-\beta_{k l} d T-\alpha \lambda D_{k l u v} \frac{\partial F}{\partial \bar{\sigma}_{u v}} \\
& =d \sigma_{k l}-\beta_{k l}^{e p} d T
\end{aligned}
$$

where

$$
d \sigma_{k l}=D_{k l i j}^{e p} d e_{i j}
$$

and

$$
d \lambda=a \cdot \lambda-\frac{\beta}{\gamma} \frac{\partial F}{\partial \bar{z}}+i i
$$

in which $d \lambda$ ' is the isothermal plastic multiplier which can be obtained from Eqs.(3.18), (3.24) and (3.37) as

$$
d \lambda^{\prime}=\frac{1}{\gamma} \frac{\partial F}{\partial \delta_{k l}} D_{k l i j}{ }^{\lambda e_{i j}}
$$

The second term in Eq. (3.41) represents the effect of thermoplastic strains and vanishes when the yield surface is independent of pressure or in the isothermal analysis.

\subsection{Field Equations in Incremental Form}

The field equations for thermoelastic consolidation, listed in chapter II, will be rewritten here in an incremental form for the thermoelastoplastic consolidation problem. They are (i) Stress-strain and strain displacement relations for an elastoplastic homogeneous solid

$$
\begin{aligned}
d \sigma_{i j} & =D_{i j k l}^{e p} d e_{k l} \\
d e_{i j} & =\frac{l}{2}\left(d u_{i, j}+d u_{j, i}\right)
\end{aligned}
$$


(ii) Equilibrium Equations for the solid fluid mixture

$$
\left(d \sigma_{i j}+d \pi \delta_{i j}-\beta e p_{d T \delta_{i j}}\right)_{, j}+d \rho f_{i}=0
$$

(iii) Darcy's law for irrotational fluid flow

$$
\mathrm{dq}_{i}=k_{i j} \mathrm{d \theta} j
$$

where

$$
d \theta_{j}=d\left(\pi, j+\rho_{2} f_{j}\right)
$$

(iv) Continuity equation

$$
d q_{i, i}=-d e_{i i}
$$

(v) Fourier's law of heat condition

$$
d h_{i}=-k_{i j} d_{j}
$$

where

$$
\mathrm{d} \phi_{j}=\mathrm{dT}, j
$$

(vi) Energy equation

$$
\mathrm{dh}_{i, i}+\rho \mathrm{C}_{\mathrm{v}} \mathrm{d} \dot{\mathrm{T}}+\beta \mathrm{ep}_{\tau_{0}} \mathrm{de}_{i i}=\mathrm{d} \rho \mathrm{r}
$$

The boundary conditions are as follows:

(a) Displacement boundary conditions

$$
d u_{i}(\underset{x}{x}, t)=d \hat{u}_{i}(\underset{x}{x}, t) \text { on } s_{1} x[0, \infty)
$$

(b) Trāction boundary conditions

$$
d t_{i}=d \hat{t}_{i}(x, t) \quad \text { on } s_{2} x[0, \infty)
$$

(c) Pore pressure boundary conditions

$$
d \pi(x, t)=d \hat{\pi}(x, t) \text { on } s_{3} x[0, \infty)
$$


(d) Fluid flow boundary conditions

$$
d Q(\underset{\sim}{x}, t)=d \hat{Q}(\underset{x}{x}, t) \text { on } s_{4} x[0, \infty)
$$

(e) Temperature boundary conditions

$$
\mathrm{dT}(\underset{\sim}{x}, t)=\mathrm{d} \hat{\mathrm{T}}(\underset{\sim}{x}, t) \text { on } \mathrm{s}_{5} \mathrm{x}(0, \infty)
$$

(f) Heat flux boundary conditions

$$
H(\underset{\sim}{x}, t)=\hat{H}(\underset{x}{x}, t) \quad \text { on } s_{6} x[0, \infty)
$$

It is worth emphasizing that in the present analysis no nonlinearity has been assumed elther in Darcy's law or in Fourier's law.

\subsection{Finite Element Formulation}

The incremental scheme and an iterative incremental scheme are presented in this section.

3.5.1 Incremental scheme

Following the thermo-elastic analysis in Chapter II, the incremental form of the equilibrium, continuity and energy equations are

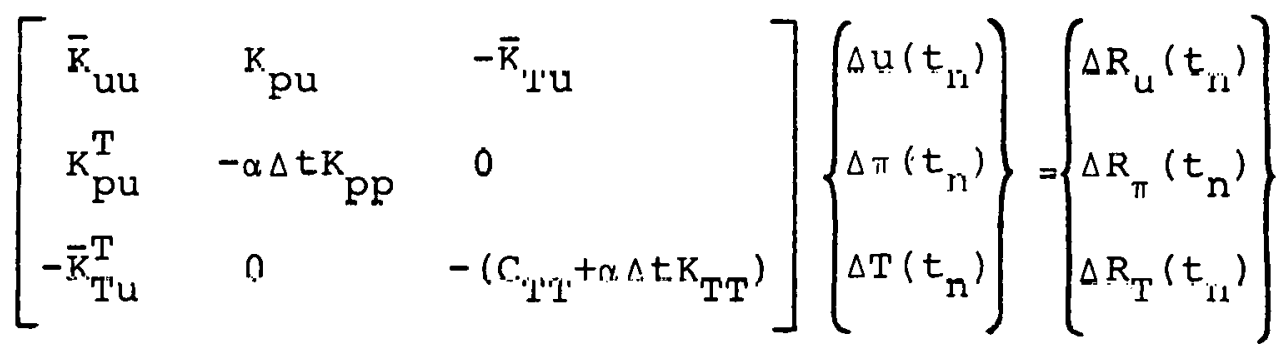

where the above matrix coefficients are the same as given by Eq.(2.85) with the exceptions

$$
\overline{\mathrm{K}}_{\mathrm{uu}}=\int \mathrm{N}_{\mathrm{e}}^{\mathrm{T}} \mathrm{D}^{e p} \mathrm{~N}_{\mathrm{e}} \mathrm{dv}
$$

and 


$$
\overline{\mathrm{K}}_{\mathrm{Tu}}=\int \mathrm{N}_{\mathrm{e}}^{\mathrm{T}} \mathrm{B}_{\mathrm{T}}^{\mathrm{ep}} \mathrm{N}_{\mathrm{T}} \mathrm{dv}
$$

The incremental scheme is the same as the one proposed by Siriwardane and Desai [51] and Prevost [44] for the isothermal elastoplastic consolidation. In this method, the loads are applied incrementally and the matrices $\overline{\mathrm{K}}_{\mathrm{uu}}$ and $\overline{\mathrm{K}}_{\mathrm{Tu}}$ are assumed to be constant throughout each increment. Essentially, the incremental procedure approximates the nonlinear problem as a series of linear problems, i.e., the nonlinearity is treated as piecewise linear. The method had been used extensively in elastoplastic analysis, Refs. $[30,32,33,66]$. The accuracy of the method can be improved by taking smaller increments of load and time or by scaling the load increment to produce plastic flow in one element per increment. Either approach requires longer computational time. The main disadvantage of the method is that the error in the solution is created at the end of each increment and accumulates as the elapsed time is increased. An incremental iterative scheme will be presented in the next section to overcome this problem.

\subsubsection{Incremental iterative scheme}

Here, the heat condition equation is decoupled from the equilibrium and continuity equations since the effect of the displacement on the temperature is neqliqible. For eash increment, the temperature solution is obtained by solving Eq. (3.62C) and dropping the $\bar{K}_{T u}^{T} \Delta u\left(t_{n}\right)$ term, i.e. 


$$
-\left(C_{T T}+\alpha \Delta t K_{T T}\right) \Delta T\left(t_{n}\right)=\Delta R_{T}\left(t_{n}\right)
$$

Then the temperature contribution to the right hand side of the equilibrium equation is considered, i.e.

$$
\left[\begin{array}{cc}
\bar{K}_{u u} & K_{p u} \\
K_{p u}^{T} & -\alpha \Delta t K_{p p}
\end{array}\right]\left\{\begin{array}{l}
\Delta u\left(t_{n}\right) \\
\Delta \pi\left(t_{n}\right)
\end{array}\right\}=\left\{\begin{array}{l}
\Delta R_{u}\left(t_{n}\right)+\bar{K}_{T u} \Delta T\left(t_{n}\right) \\
\Delta R_{\pi}\left(t_{n}\right)
\end{array}\right\}
$$

So far the scheme is still incremental and iterations are performed during each increment using

$$
\left[\begin{array}{cc}
\bar{K}_{u u} & K_{p u} \\
K_{p u}^{T} & -\alpha \Delta t K_{p p}
\end{array}\right]\left\{\begin{array}{c}
\Delta u\left(t_{n}\right)_{1} \\
\Delta \pi\left(t_{n}\right)_{i}
\end{array}\right\}=\left\{\begin{array}{l}
\phi_{u}\left(t_{n}\right)_{i} \\
\phi_{\pi}\left(t_{n}\right)_{i}
\end{array}\right\}
$$

where $i=1,2, \ldots$ is the number of iterations and

$$
\begin{aligned}
\phi_{u}\left(t_{n}\right)_{i} & =M_{1}\left(t_{n}\right)+M_{3}\left(t_{n}\right)-\int_{v} N_{e}^{T}\left(\sigma\left(t_{n}\right)_{i}+\pi\left(t_{n}\right)_{i}\right) d v+\bar{K}_{T u}^{T}\left(t_{n}\right) \\
\phi_{\pi}\left(t_{n}\right)_{i} & =K_{p u}^{T} u\left(t_{n-1}\right)+(1-\alpha) \Delta t K_{p p}^{\pi}\left(t_{n-1}\right) \\
& +\alpha \Delta t\left(M_{2}\left(t_{n-1}\right)-M_{4}\left(t_{n}\right)\right)+(1-\alpha) \Delta t\left(M_{2}\left(t_{n-1}\right) M_{4}\left(t_{n-1}\right)\right. \\
& -K_{p u}^{T} u\left(t_{n}\right)_{i-1}+\alpha \Delta t K_{p p^{\pi}}\left(t_{n}\right)_{i-1}
\end{aligned}
$$

Iterations are stopped when

$$
\frac{\| \Delta U\left(t_{n}\right)}{\left\|U\left(t_{n}\right)\right\|} \times 100 \leq \varepsilon
$$

where $U^{i T}\left(t_{n}\right)=\left\{u\left(t_{n}\right), \pi\left(t_{n}\right)\right\}$

and $\varepsilon$ is an allowable tolerance.

Finally, stiffnesses are allowed to change during each increment. It is advisable to keep stiffness constant at the 
onset of loading as well as prior to failure if a perfectly plastic model is adopted to avoid ill conditioning. Keeping Eq. (3.63) with constant stiffness throughout the loading program requires many iterations. The number of these iterations can be reduced by modifying the load vector to account for plastic loading, e.g. the initial stress method in elastoplastic analysis $[1,37,38,70]$. 
CHAPTER IV

NUMERICAL RESULTS

\subsection{Introduction}

Based on the preceding finite element formulations, several special purpose computer codes were developed for evaluating the isothermal, thermal, elastic and alaston]astic consolidation responses. In this chaptcr, validation nf the. developed codes is presented along with applications to a field problem applicable to underground coal conversion.

\subsection{Code Validation}

'lie computer codes developed in this study are validated for both the thermo-elastic and thermo-elastoplastic analysis.

\subsubsection{Elastic analysis}

In the early stages of this study, the code consol was developed using the formulation of Sandhu and wilson [49] to investigate isothermal plane strain consolidation problems and the 8-4 and 8-8 isoparametric elements, Ref. [53]. An extension of this program, TCONSL, was then applied to solve thermoelastic plane strain consolidation problems using the formulation $\mathrm{Eq} \cdot(2.93)$ and the 8-4-4 and the 8-8-8 isopardmetric elements in Figs. $(2.1,2.2)$. 
To validate the finite element programs, a problem with known analytical solution has to be selected and compared. Unfortunately, there are no available analytical or numerical solutions even for a simple thermoelastic consolidation problem. Analytical and finite element solutions to Terzaghi's one-dimensional isothermal consolidation problem can be found in Ref.[53]. These solutions were used to validate the programs CONSOL and TCONSL. The closed form solution for a simple one-dimensional heat conduction problem, found in Ref.[9], was used to validate TCONSL. As an additional step, the solutions associated with thermoelastic consolidation (displacement, pressure and temperature) from TCONSL are compared qualitatively with the solutions for isothermal consolidation (displacement and pressure) and heat conduction (temperature). The problem selected is a generalization of Terzaghi's one-dimensional isothermal consolidation problem subjected to thermal loading in addition to the tradionally applied surface tractions. 'l'he selected problem is a linear elastic saturated soil column under constant surface tractions and constant surface temperature, Fig.(4.1). The problem is solved with the following numerical values: $L=7, E=6000, \nu=.4$, $\mathrm{K}=4 \times 10^{-6}, \mathrm{k}=.2, \rho \mathrm{C}_{\mathrm{V}}=40, \tau_{\mathrm{o}}=100, \bar{u}=.3 \times 10^{-6}$. A surface traction of unity is applied together with a surface temperature $\hat{\mathrm{T}}=50$ and initial temperature $T_{0}=0$. The soil column is assumed to be insulated and sealed everywhere, except at the top surface. From this data the following problems are analyzed:. 
(i) Thermoelastic consolidation problem (TCONSL)

(ii) Isothermal consolidation problem (TCONSL, CONSOL, analytical and numerical solutions from Ref.[53])

(iii) Heat conduction problem (TCONSL and analytical solution of Ref. [9]).

It shoula be noted TCONSL is used in problem (ii) by prescribing the temperature to be zero at all nodes and is used in (iii) by prescribing the displacement and pore pressure to be zero at all nodes. The two special interpolation schemes mentioned earlier are used in the analysis, but the presented results are obtained by using the 8-4-4 element. The mesh used in the analysis is shown in Fig.(4.2). Fig. (4.3) illustrates pressure and temperature prof1les at two different time steps $A$ and $B$. In this figure, the finite element profiles for the thermoelastic consullation problem (using TCONSL) and the heat conduction problem (using TCONSL) almost concine and are in yuod agreement with lle analytical solution for the heat conduction problem in Ref.[9]. This indicates list the energy equation can be decoupled from the cquiliurium and continuity equations, similar to the clasaical uncoupled thermoelastic theory, Ref.[7]. The finite element pressure profiles for the thermoelastic consolidation problem (using TCONST.) are slightly less than the isothermal pressure response values (using TCONSL and CONSOL) which are in good agreement with Sandhu's solution [53]. This difference is due to thermal expansion which acts as an unloading mechanism 
for the surface tractions. The surface settlement history for the thermal and isothermal consolidation is illustrated in Fig.(4.4). In this figure, the thermal expansion reduces the thermoelastic consolidation settlement (using TCONSL) in comparison with the isothermal consolidation settlement (using TCONSL and CONSOL) which are in very good agreement with the results of Sandhu [53]. Furthermore, the steady state response with thermal effects is reached after a longer elapsed time:

\subsubsection{Efficiency of the Discretization Schemes}

The effect of spatial and temporal discretizations on the solution of the thermoelastic consolidation problem is investigated by analyzing the same one-dimensional problem in Fig.(4.1). The responses obtained from the two element schemes in Figs.(2.1) and (2.2) are compared for different values of $\alpha$. For both the elements, the following temporal partitioning $I$ is used; 10 steps of $\Delta t=.01$ over $[0, .1] ; 10$ steps of $\Delta t=.1$ over $[.1,1.1] ; 10$ steps of $\Delta t=10$ over [1.1, 101.1]; 10 steps of $\Delta t=100$ nver $[101.1,1101.1] ; 20$ steps of $\Delta t=1000$ over $[1101.1,21101.1]$. This same temporal discretization gave good agreement with the analytical solution to the isothermal consolidation [53].

Comparing the solutions of the aforementioned problem (Fig.(4.1)), using the two spatial interpolation schemes, it was found that when $\alpha=.5$, and for small values of the time variable, the 8-4-4 element gave larger oscillations in 
pressure and temperature profiles in a region very close to the loading surface. Also, the 8-8-8 element showed an oscillatory pressure response throughout the vertical domain for all times, with no oscillations in the temperature profile. When $\alpha$ is increased to .875 , the oscillations in the pore pressure associated with the 8-8-8 element vanish without a significant effect on the temperature distribution. The initial oscillations associated with the 8-4-4 element are reduced. Upon inorcasing the time variable, the two spatial interpolation schemes yield almost identical distributions for both the pressure and temperature profiles, Fig.(4.5a) through Fig. (4.5h).

The solution of the problem, in fact, depends not only on the choice of $\alpha$ but also on the size and the change in $\Delta t$. The sensitivity of the solution to the sudden change in $\Delta t$ is examined. It should be noted that, for the previous comparison, $\Delta t$ had heen shanged from .01 to 1000 in five stages, will a ratiu uf change equal to $1: 10$, except the ratio is 1:100 at $t=1.1$. To illustrate the effect of this sudden change in $\Delta t$, another time partitinn $I I$ is uecd. In thib new time partition, the ratio $1: 100$ is eliminated as follows: 10 steps of $\Delta t=.01$ over $[0, .1] ; 10$ steps of $\Delta t=.1$ over $[.1$, $1.1] ; 10$ steps of $\Delta t=1$ over $[1.1,11.1] ; 9$ steps of $\Delta t=10$ over $[11.1,101.1] ; 10$ steps of $\Delta t=100$ over $[101.1,1101.1]$; 20 steps of $\Delta t=1000$ over [1].01.1,21101.1]. A comparison between the two time partitions is performed using the 8-8-8 
element with $\alpha=.5$. It is found that the error associated with the sudden change in $\Delta t$ in scheme $I$ dies out in a few steps. As a increases to .875 , the sudden change in $\Delta t$ causes no difficulty, Figs.(4.6a) through (4.6c). Finally, it is found that the pore pressure is more significantly affected by the sudden change in $\Delta t$ than the temperature. Increasing a from .5 to .875, for the same spatial discretization and the same temporal partitioning, is found to have a negligible effect on the surface settlement. In addition, the settlement is slightly affected by the sudden change in $\Delta t$. However, the two spatial interpolation schemes yield slight differences in the surface settlement especially when $t .1$, (Table 4.1).

To increase the efficiency of the program TCONSL, a modified version, HCONSL, has been developed. In this program, the energy equation is decoupled from the equilibrium and continuity equations. As mentioned earlier, this decoupling is desirable since the temperature solution for thermoelastic consolidation and heat conduction are identical for this problem, Fig.(4.1). The approach is very slmilar to the one discussed in Chapter III. When HCONSL is used to solve the problem in Fig.(4.1), the same results as those from TCONSL are obtained. However, a tremendous saving in CPU time $(19.43 \mathrm{sec}$ for HCONSL versus $29.78 \mathrm{sec}$ for TCONSL on the OSU AMDAHL 470 system) is evident. 


\subsubsection{Elastoplastic Analysis}

The program PCONSL is an extension of HCONSL to solve elastoplastic plane strain thermal consolidation problems, using the developed formulation, Eq. (3.63) and the 8-8-8 and 8-4-4 elements. Again, there are no available solutions even for simple thermoelastoplastic consolidations. The code is checked by solving the following problems:

(i) A steady state plane strain thick circular cylinder subjected to internal pressure, Fig.(4.7). The material is modeled as an elastic-perfectly plastic material obeying the Von Mises yield criterion. The code PCONSL is used in this problem by prescribing the pressure and temperature to be zero at all nodes. The load is applifer incremently and the inner surface displacement versus the applied loading is shown in Fig. (4.8). Fond agreellent can be observed between the solutions using PCONSL and the analytical and numerical solulions in Ref. [40].

(ii) Thermoelastic consolidation problem: the continuum in prublem $\mathrm{F} 1 \mathrm{~g} \cdot(4.1)$ is remodeled as an elastic-work hardening material obeying the cap yield oriteria in Appendix B. To obtain the elastic response using PCONSL, the yield stress is assumed to be relatively large. The previoue elastir, themild and flow properties are assumed together with cohesion, $c=30$, internal friction angle, $\phi=30^{\circ}$, cap ratio, $R=3.5$, cap hardening constant, $\mathrm{G}=.0007$ (see Appendix B). The response is the same as 
that obtained by TCONSL and HCONSL, Figs. (4.3) and $(4.4)$.

(iii) Thermoelastic-plastic consolidation problem: This problem is identical to (ii) except the yield stress is lower. Four different cases are analyzed.

A. Elastic analysis

B. Elastoplastic analysis with $\mathrm{c}=.9$

C. Elastoplastic analysis with $c=.85$

D. Elastoplastic analysis with $c=.7$.

The surface settlement history and progression of plastic zone are illustrated in Fig.(4.9). As the elapsed time is increased, plastic flow accounts for an increasing surface settlement which increases drastically for case D. The temperature unloading is elastic in the context of the theory of plasticity. This causes the unloading portion of curves $B$ and $C$ to be almost parallel to curve $A$. In case D, plastic flow occurs very tally aild lemperature unloading oan not resist plastification. The surface displacement, therefore, 1ncreases in an expunential fashion. Rcgarding the progress of plastic flow, it should be noted that plastification starts from the bottom in case $B$ and from the top surface in case D. This is mainly due to the critical value of the deviatoric stress, which is reached at an earlier time in the 
upper element, for case $D$, while it reaches the lower element at a later stage in case B. Pore pressure and temperature profiles for cases $A$ and $C$ are illustrated in Fig.(4.10a-h). The elastoplastic analysis yields larger pressure response due to the increase in the volumetric strain over the elastic analysis. However, temperature profile is the same in the two caps due to decoupling the heat equation.

\subsection{Field Appliaation}

Elastic and elastoplastic thermal consolidation analyses are conducted for the Centralia Coal gasification site. Figure (4.11) illustrates the stratification and the finite element mesh used in the analysis. The material properties are listed in Table 4.2. These materials were modeled as elastic work hardening materials obeying the elliptic cap yield criteria in Appendix B. The cavity temperature history is shown in Fig.(4.12). The selllement history and the progression of plastic zones are illustrated in Fig.(4.13). In this figure, the temperature rise accounts for reducing the settlement in both elastic and plastic cases during the first 13 months. However, gravity loading dominates the settlement in the elastic and elastoplastic problems. Upon reducing cavity temperature, the settlements start increasing with the notable difference between the elastic and elastoplastic analysis. Progression of plastic zones is also illustrated in Fig. (4.13). Plastification starts as early as $t=.40$ month, and 
increases with time till $t=1$ month, with plastic zones propagated around the cavity. When the cavity temperature is reducing, the plastic zones start to propagate again. 
TABLE 4.1 Vertical Surface settlement History Using $\alpha=.875$ for the $8-8-8$ and the 8-4-4 Element

\begin{tabular}{lll}
\hline Time & the $8-8-8$ element & the $8-4-4$ element \\
.02 & $.20252 \times 10^{-5}$ & $.30899 \times 10^{-5}$ \\
.1 & $.53216 \times 10^{-5}$ & $.53273 \times 10^{-5}$ \\
.5 & $.12327 \times 10^{-4}$ & $.12037 \times 10^{-4}$ \\
21.1 & $.78613 \times 10^{-4}$ & $.791401 \times 10^{-4}$ \\
1101.1 & $.17709 \times 10^{-3}$ & $.17802 \times 10^{-3}$ \\
3101.1 & $.29569 \times 10^{-3}$ & $.29713 \times 10^{-3}$ \\
17100.1 & $.30271 \times 10^{-3}$ & $.30334 \times 10^{-3}$ \\
21101.1 & $.30106 \times 10^{-3}$ & $.30095 \times 10^{-3}$ \\
\hline
\end{tabular}


TABLE 4.2 Material Properties for the Centralia Coal Gasification Problem

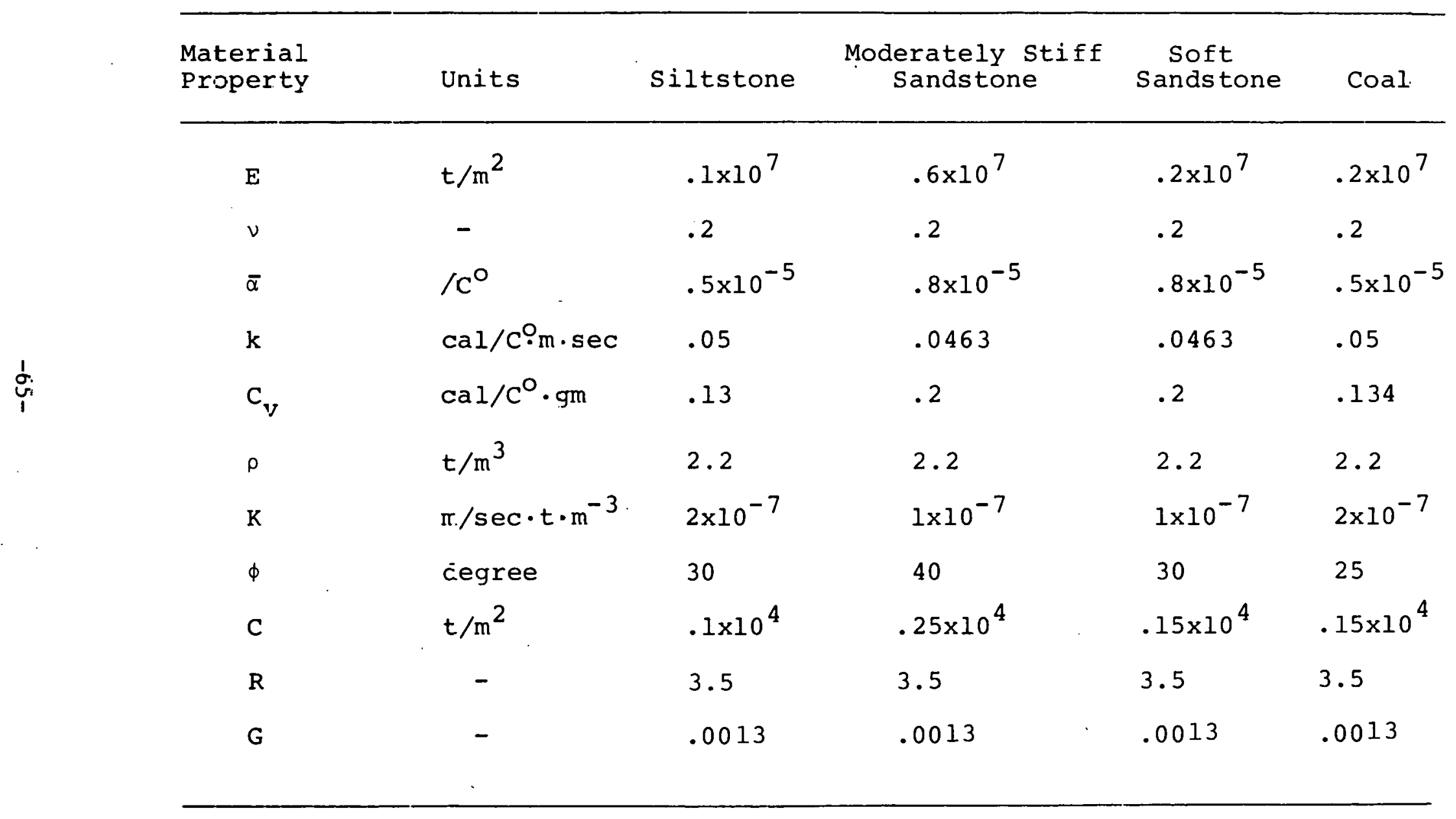




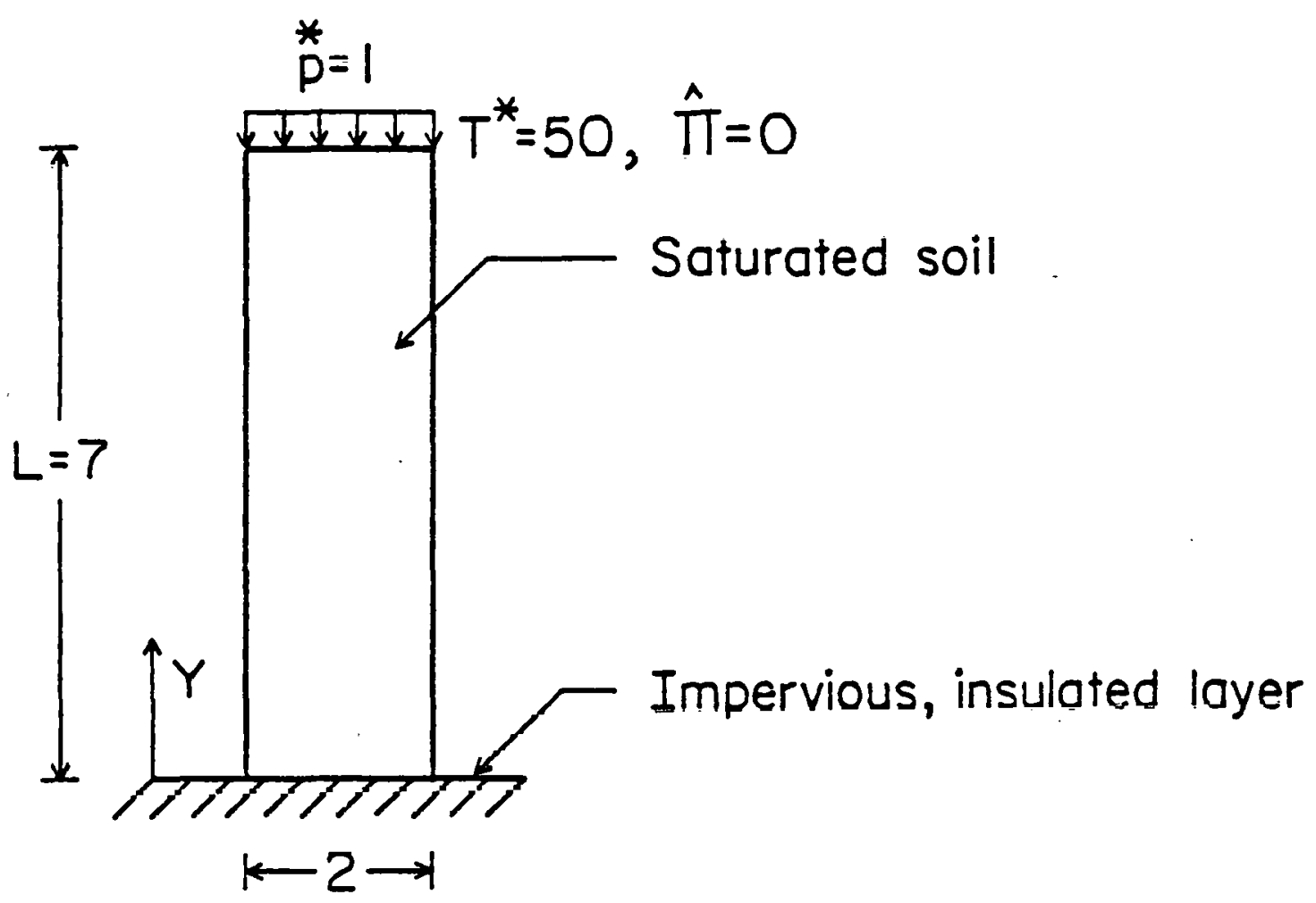

Figure 4.1. One-Dimensional Thermoelastic Consolidation 


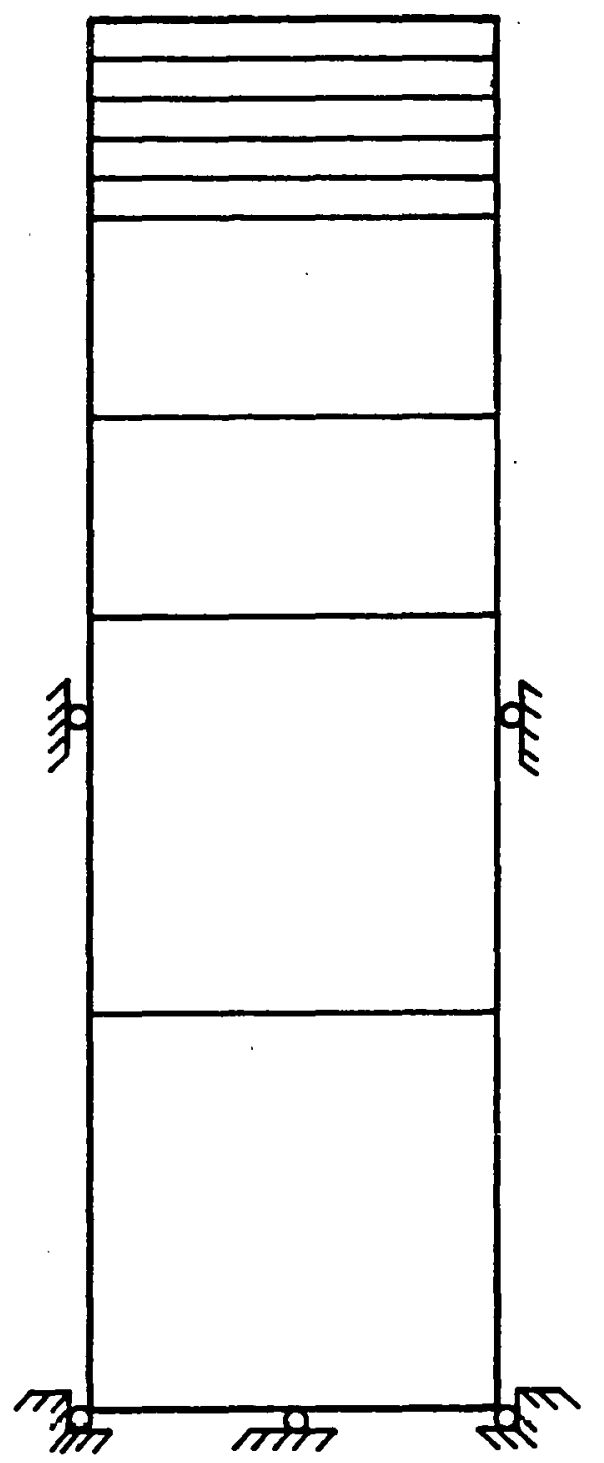

Figure 4.2. Mesh for the 8-4-4 and the 8-8-8 Element

$-67-$ 


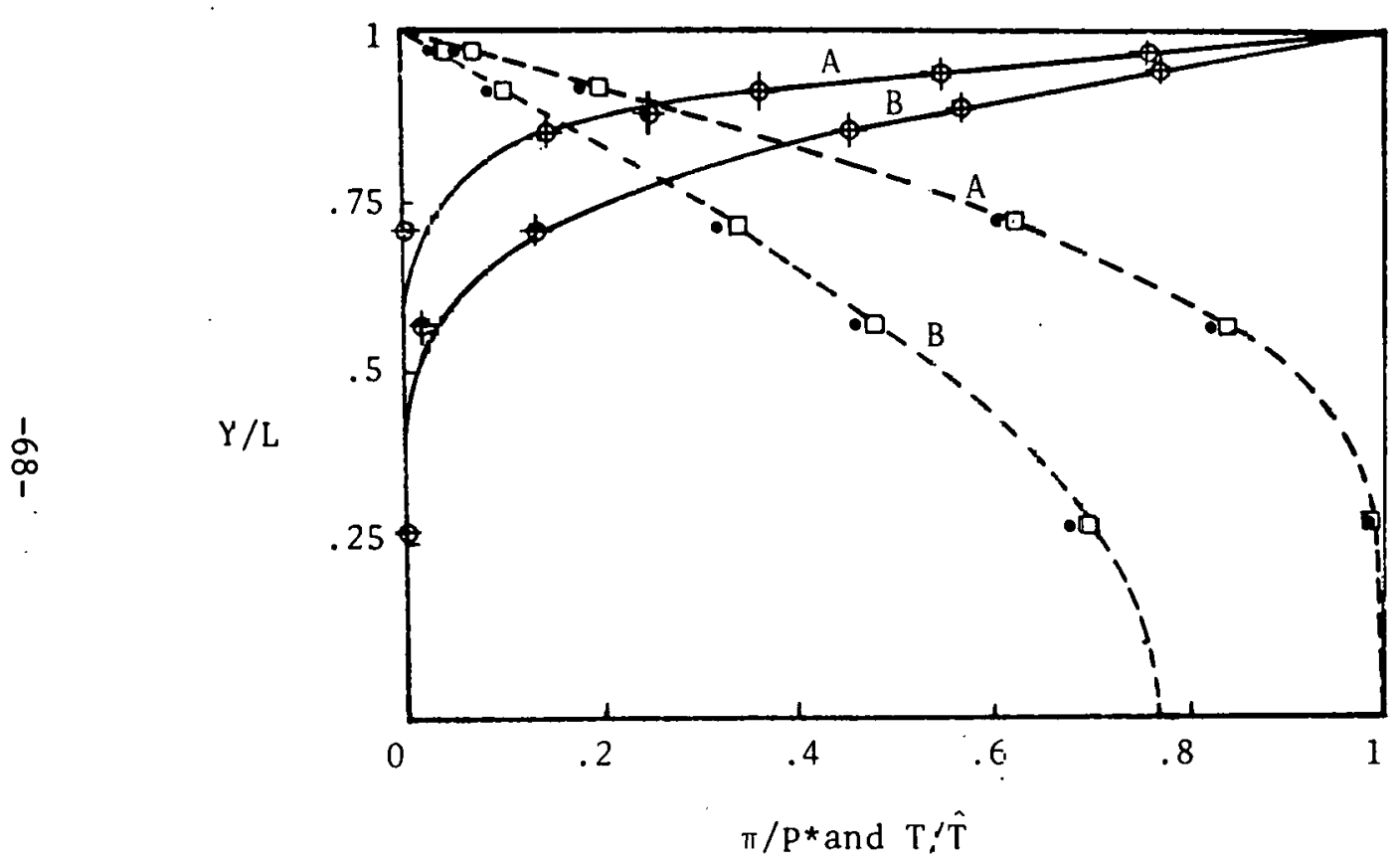

$\mathrm{T} / \hat{\mathrm{T}}$ p1ots;

- Series solution

(Ref.[9])

++ Present FEM heat conduction solution

- o Present FEM thermoelastic consolidation solution

$\pi / \mathrm{P}^{*} \mathrm{p}$ lots;

- Isothermal consolidation (Ref.[52])

D Isothermal consolidation (present solution)

- Thermoelastic consolidation (present solution)

Figure 4.3. Pressure and Temperaiure Profiles at Twa Different Time Steps: $\frac{k t}{\rho C_{v} L^{2}}=\left\{\begin{array}{l}0.005 \text { for case A } \\ 0.02 \text { for case } B\end{array}\right.$ 


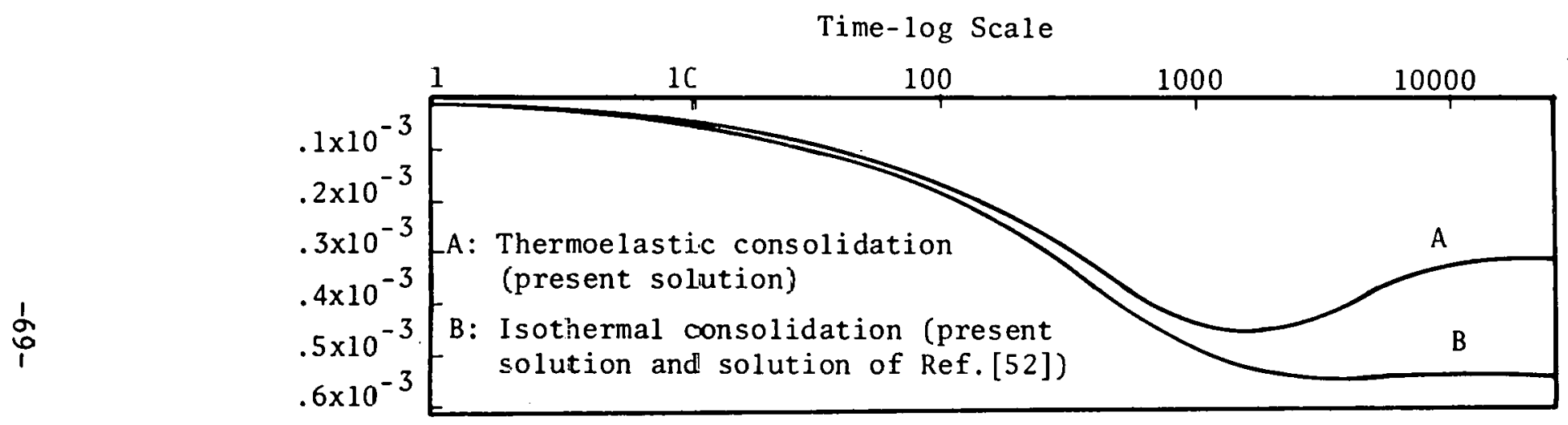

Figure 4.4. Surface Settlement History for Isothermal and Thermal Consolidation 


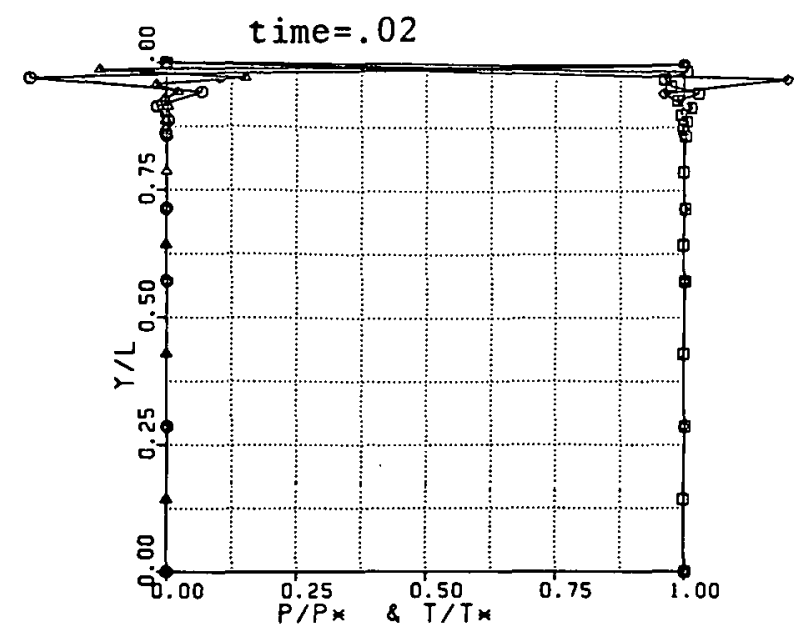

(a)

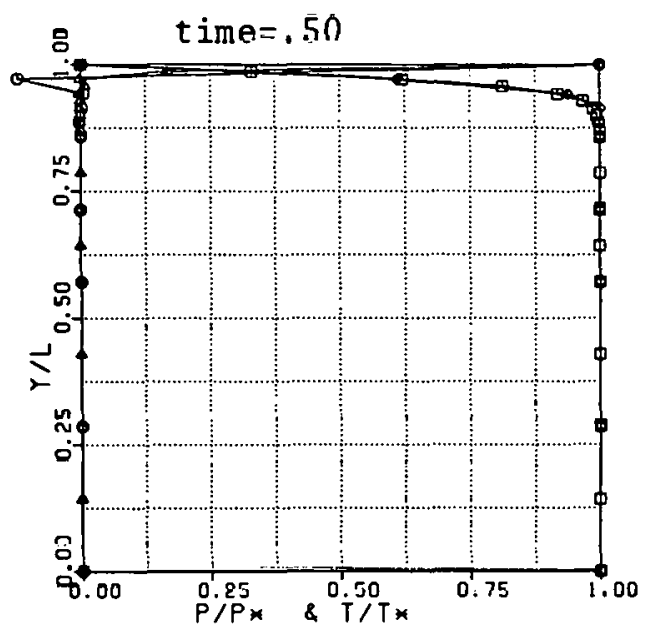

(c)

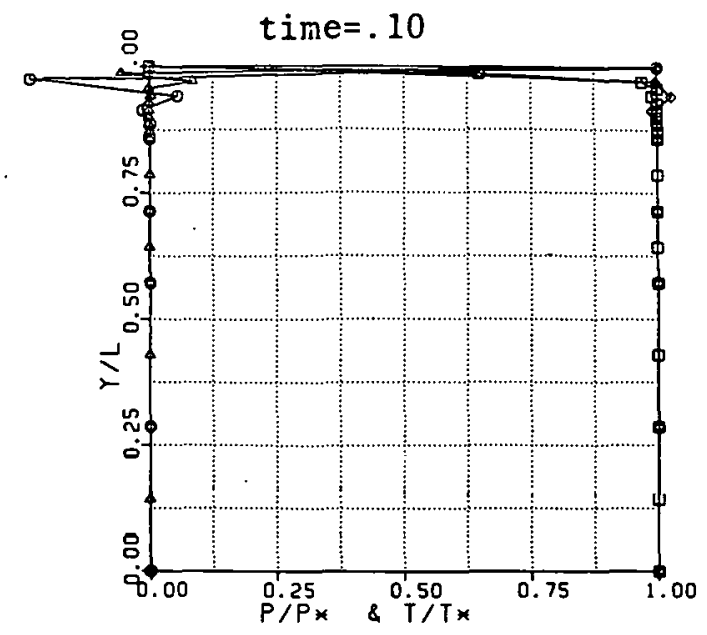

(b)

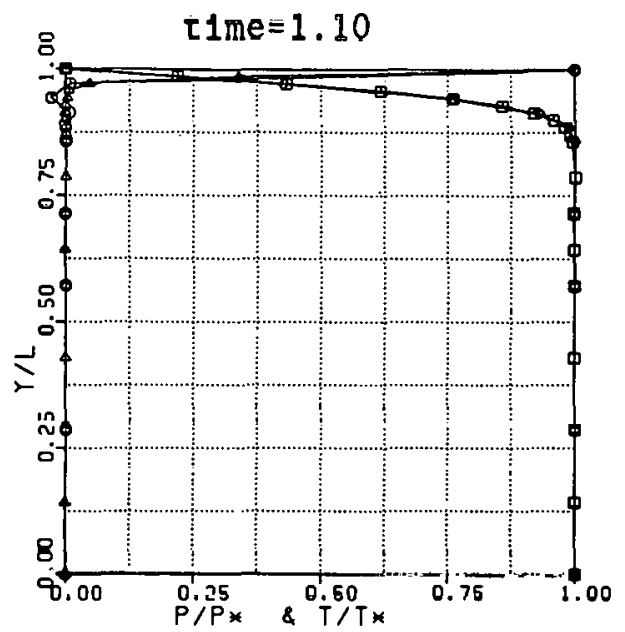

(d)

0. pressure using $8-8-8$ element, $\alpha=.875$, time partitioning I

$\triangle \quad$ temperature using $8-8-8$ element, $\alpha=.875$, time partitioning I

- pressure using 8-4-4 element, $\alpha=.875$, time partitioning I

- temperature using 8-4-4 element, $\alpha=.875$, time partitioning I

Figure 4.5a through 4.5d. Effect of Spatial Discretization on Pressure nnd Temperature Profile 


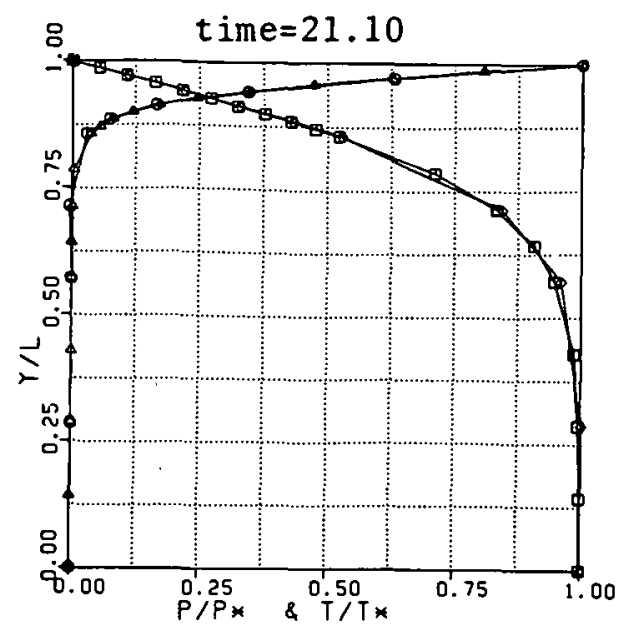

(e)

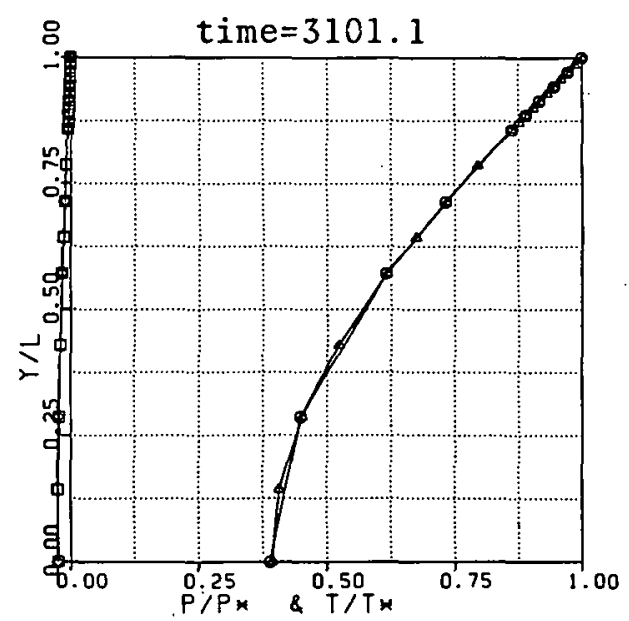

(g)

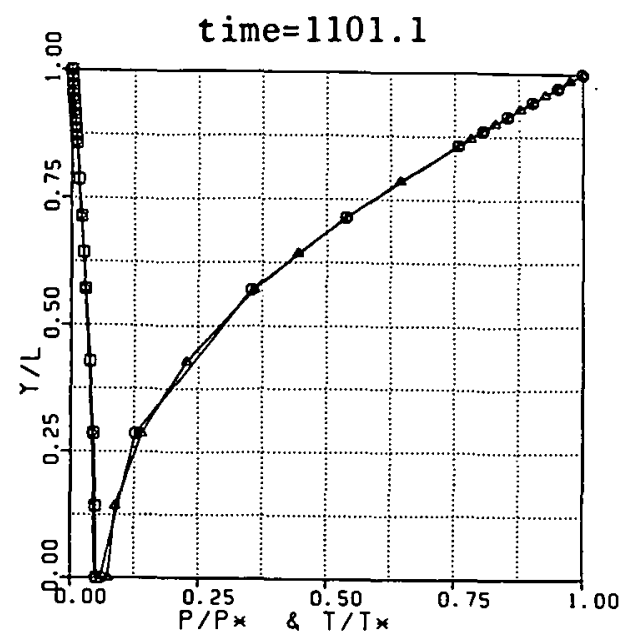

(f)

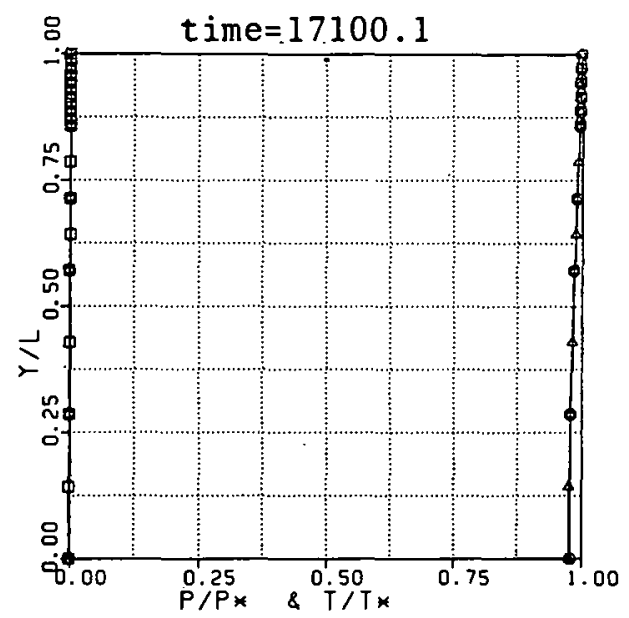

(h)

pressure using $8-8-8$ element, $\alpha=.875$, time partitioning I
$\Delta \quad$ temperature using $8-8-8$ element, $\alpha=.875$, time partitioning T
$\diamond \quad$ pressure using $8-4-4$ element, $\alpha=.875$, time partitioning I
0 temperature using $8-4-4$ element, $\alpha=.875$, time partitioning I

Figure $4.5 e$ through $4.5 \mathrm{~h}$. Effect of Spatial Discretization of Pressure and Temperature Profiles 


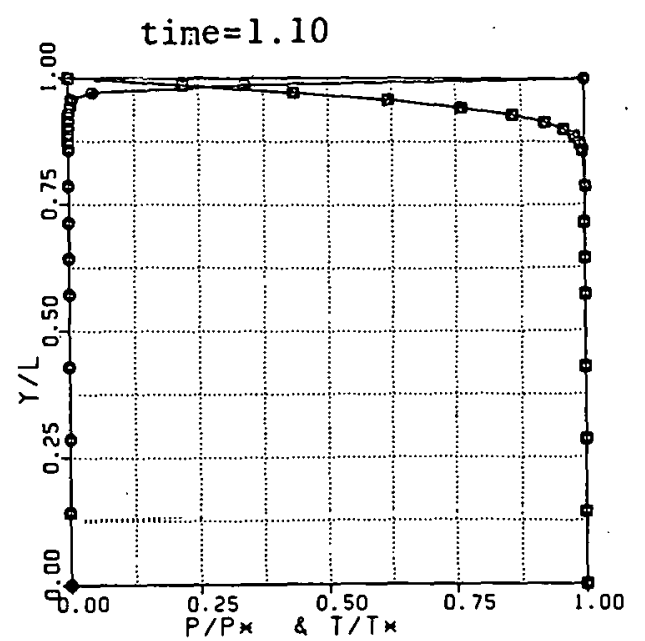

(a)

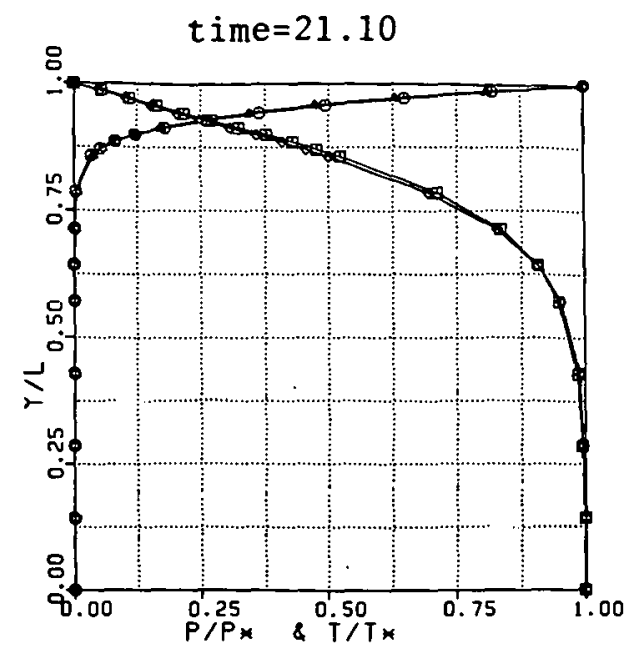

(b)

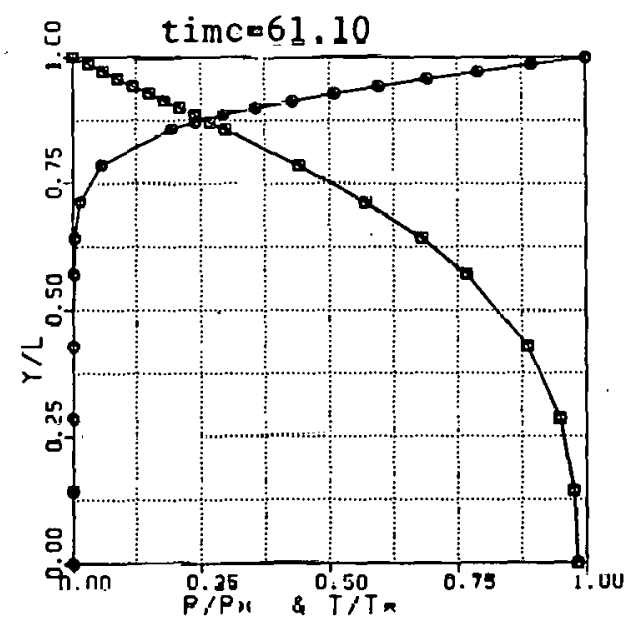

(c)

D pressure using 8-8-8 element, $\alpha=.875$, time partitioning I

$\triangle \quad$ temperature using 8-8-8 element, $\alpha=.875$, time partitioning I

- pressure using 8-8-8 element, $\alpha=.875$, time partitioning II

- temperature using 8-8-8 element, $\alpha=.875$, time partitioning II

Figure 4.6a through 4.6c. Effect of Temporal Partitioning on Pressure and Temperature "rofiles 


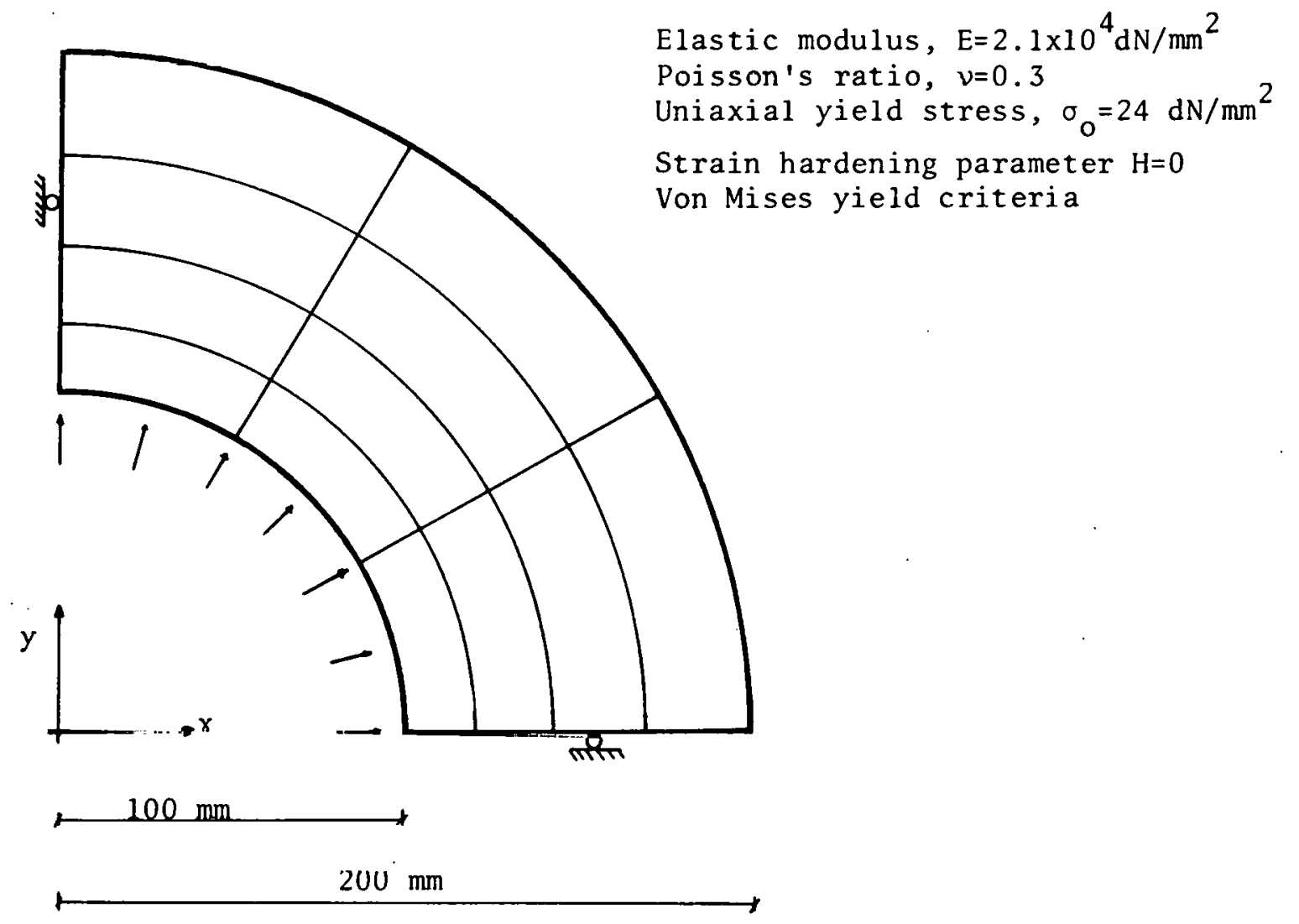

Figure 4.7. Thick Circular Cylinder Subjected to Internal Pressure 


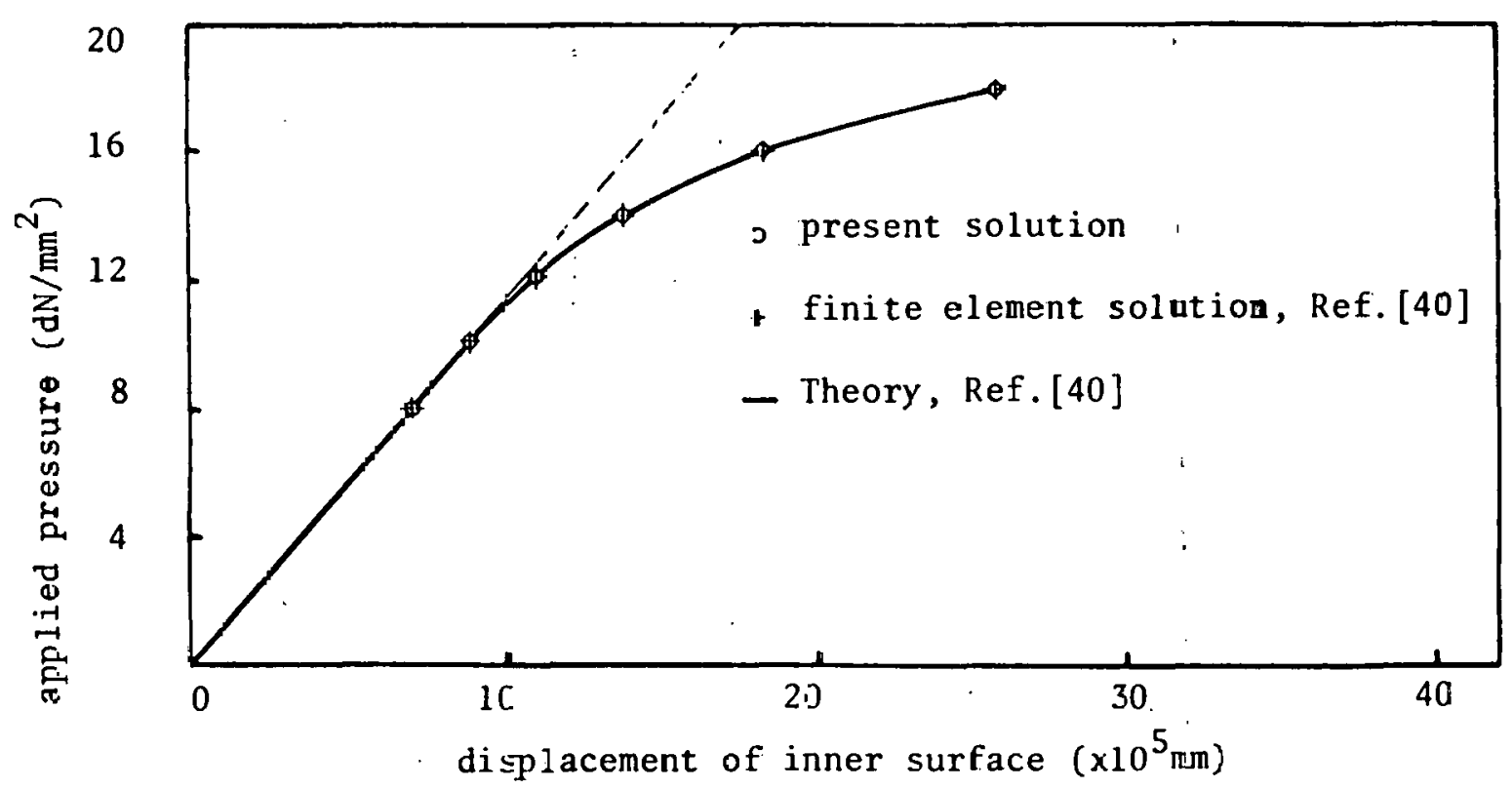

Figure 4.8. Inner Surface Displacenent with Increasing Pressure 
Time- $\log$ Scale
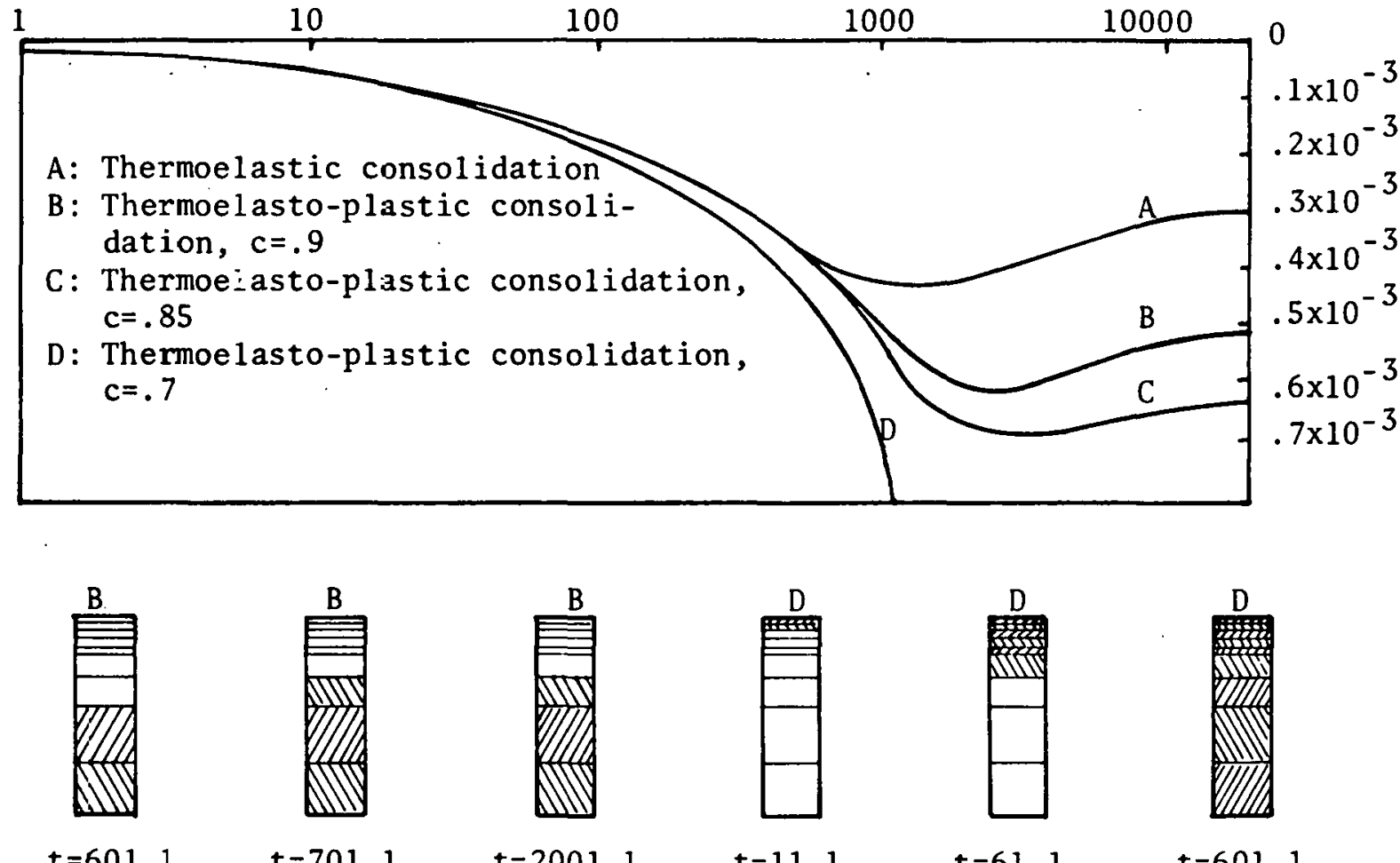

$t=601.1$

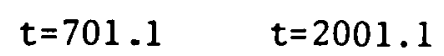

$t=11.1$

$t=61.1$

$t=601.1$

Figure 4.9. Surface Settlement History and Progression of Plastic Zones. (Hatched area implies yielded element.) 


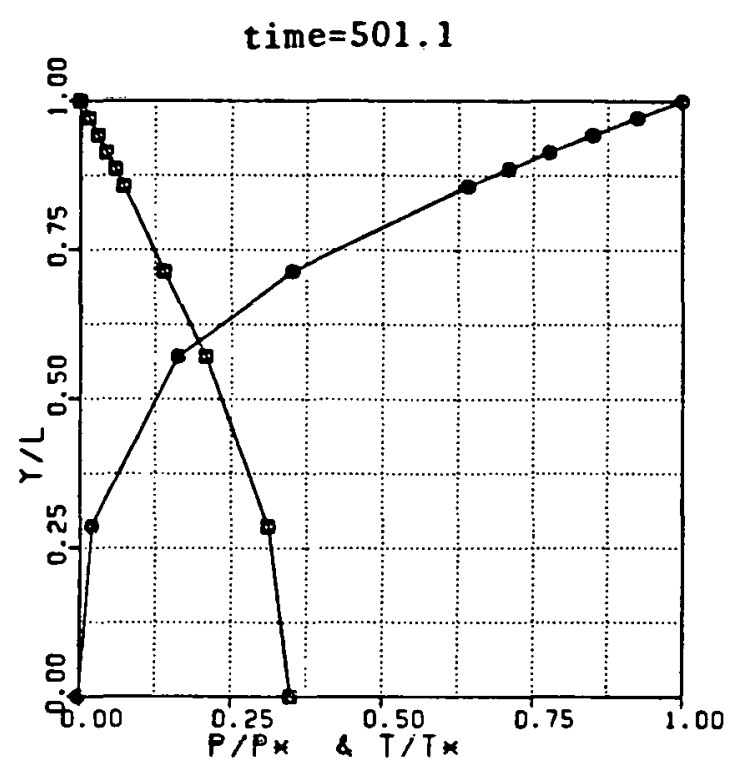

a

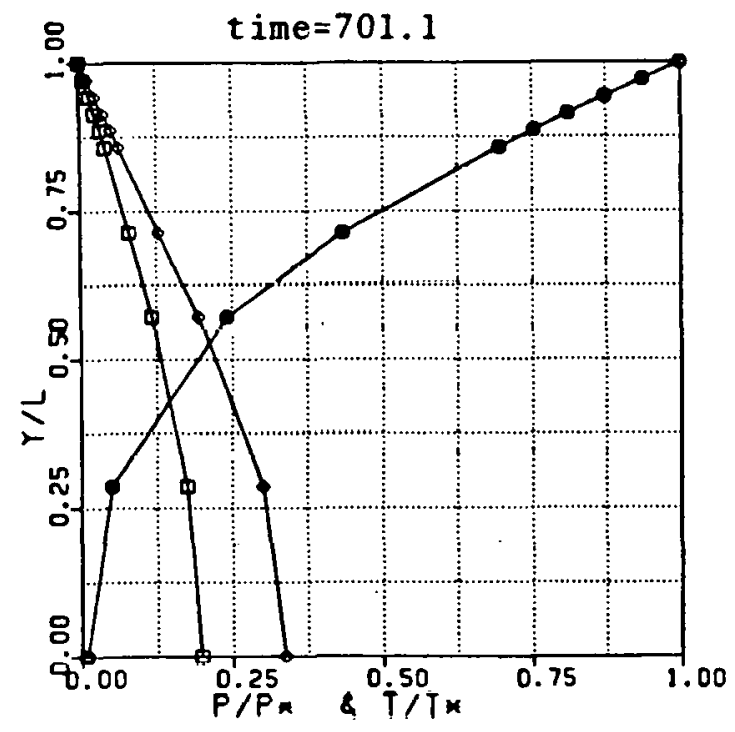

$\square$ Pressure, elastic analysis
$\triangle$ Temperature, elastic analysis
$\odot$ Pressure, elasto-plastic analysis
O Temperature, elasto-plastic analysis

Figure 4.10a-d: Elastic and Elasto-plastic Pressure and Temperature Profiles

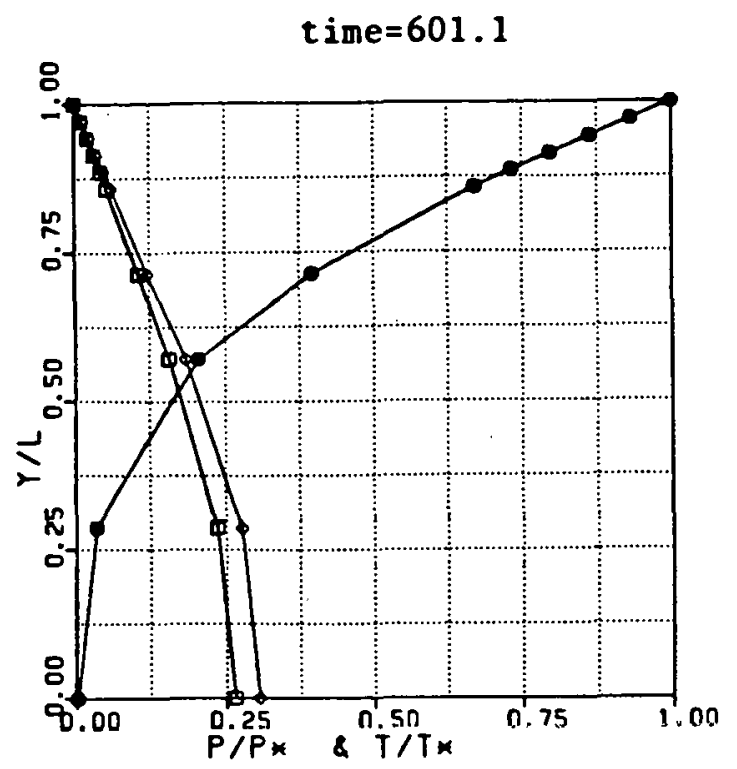

$\mathbf{b}$

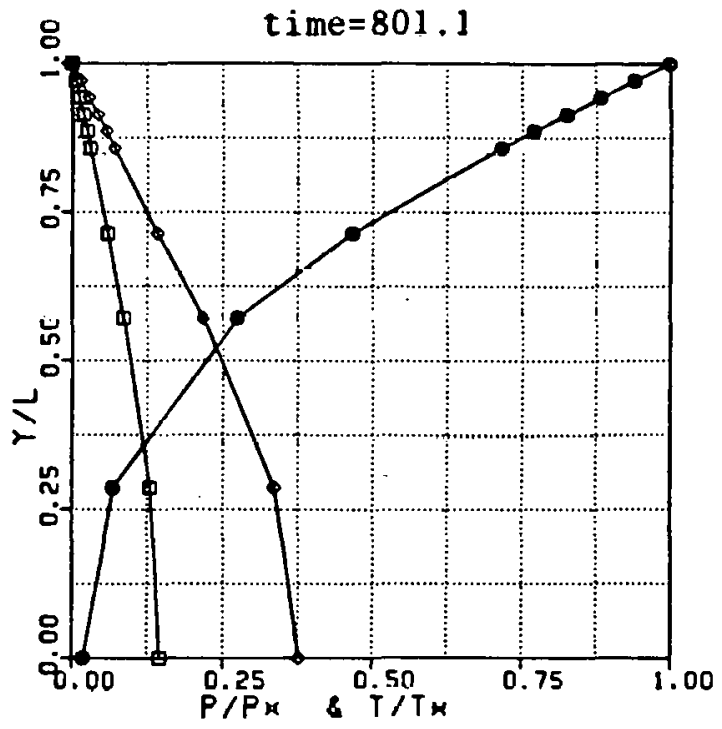

d 


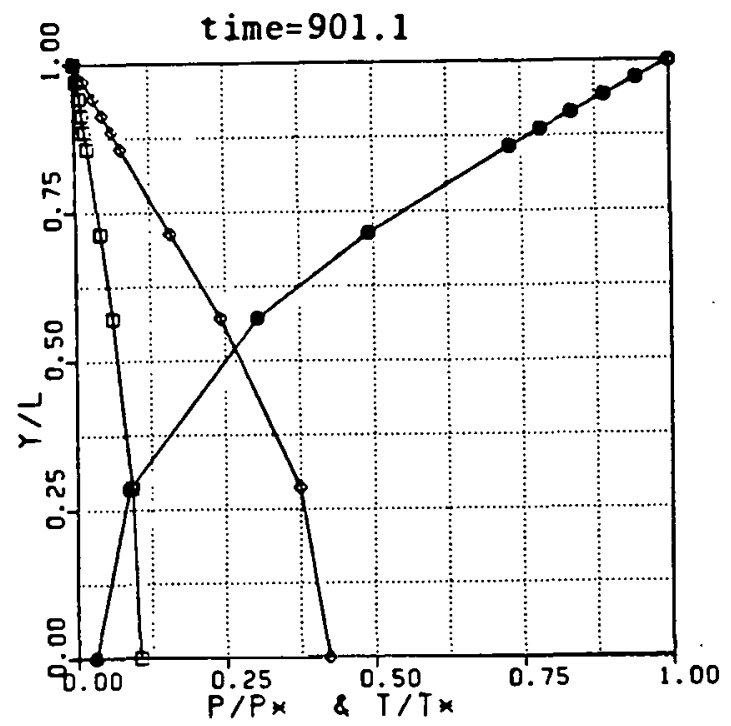

e

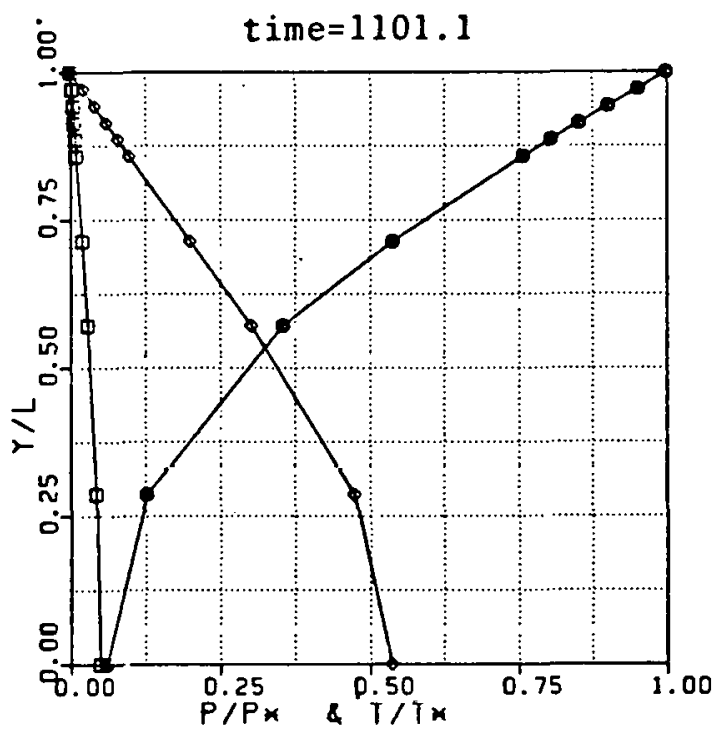

$\mathrm{g}$

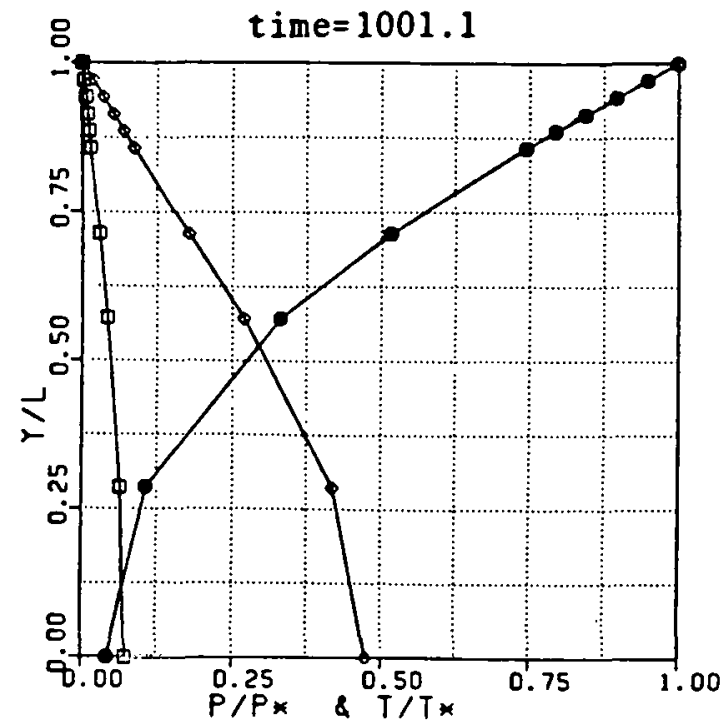

$\mathbf{f}$

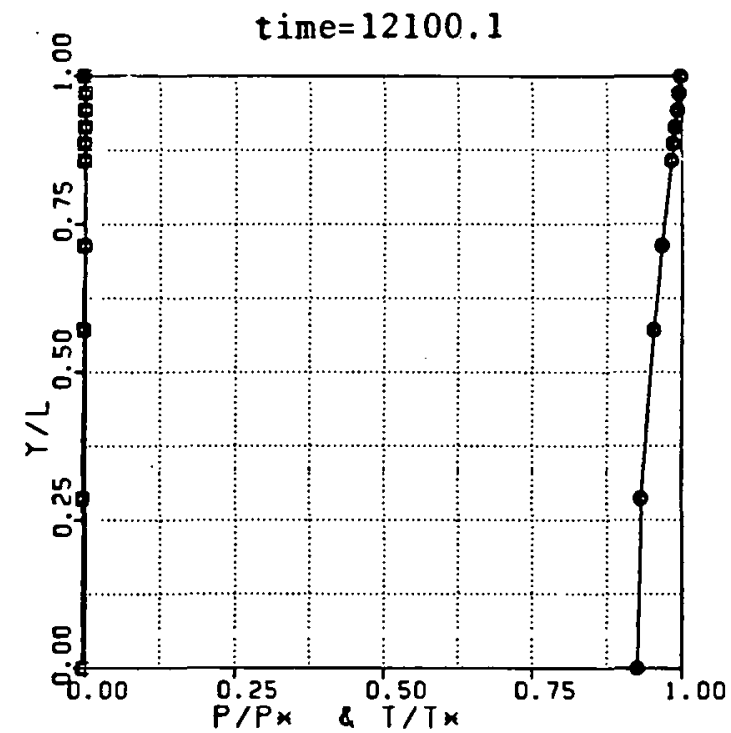

h

$\square$ Pressure, elastic analysis

$\triangle \quad$ Temperature, elastic analysis

- Pressure, elasto-plastic analysis

( ) Temperature, elasto-plastic analysis

Figure 4.10e-f: Elastic and Elasto-plastic Pressure and Temperature Profiles 


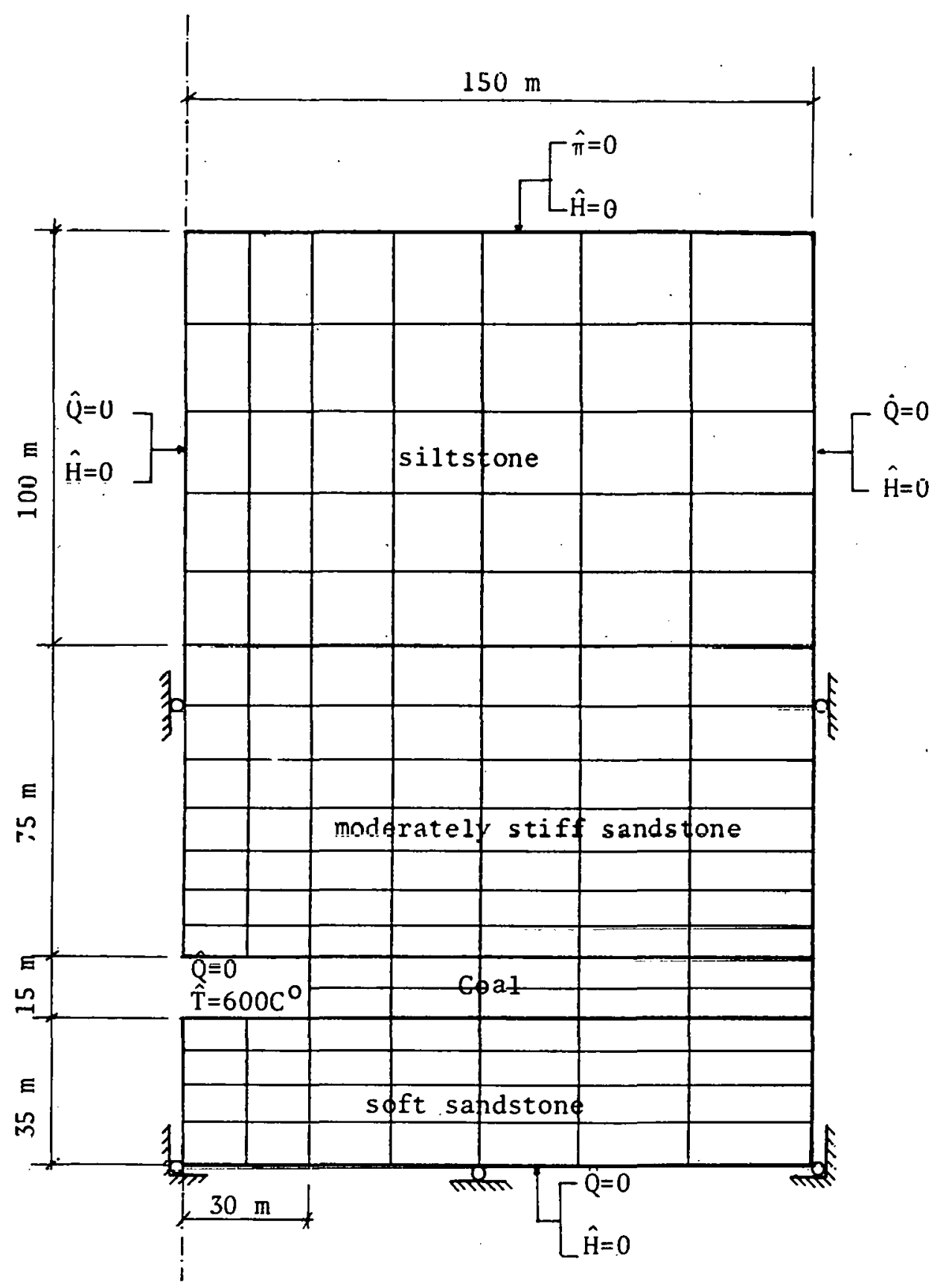

Figure 4.11. Finite Element Mesh and Strata Description for Centralia Coal Gasification Site 


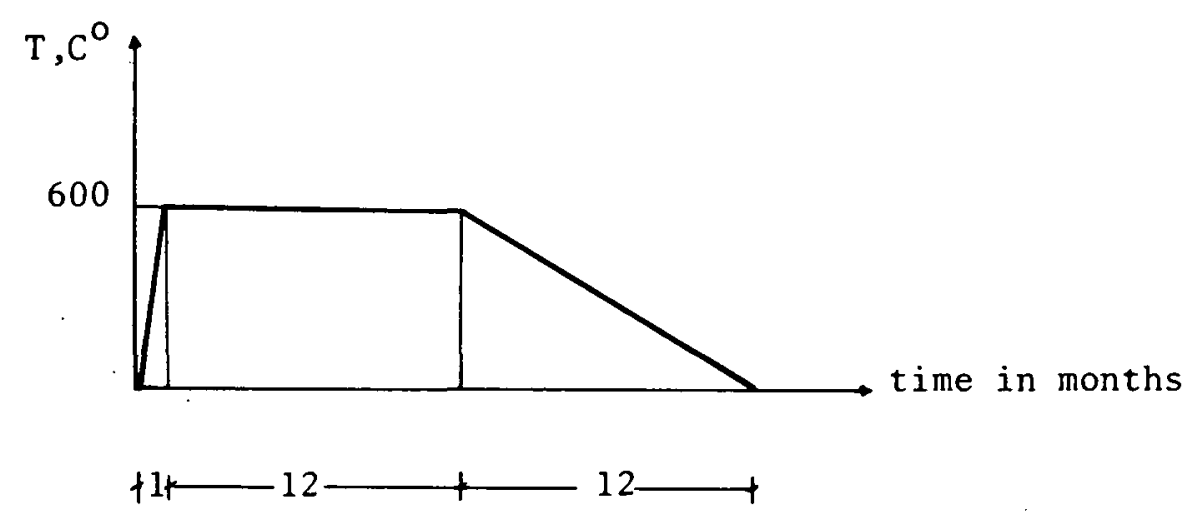

Figure 4,12. Cavity Temperature versus Time 


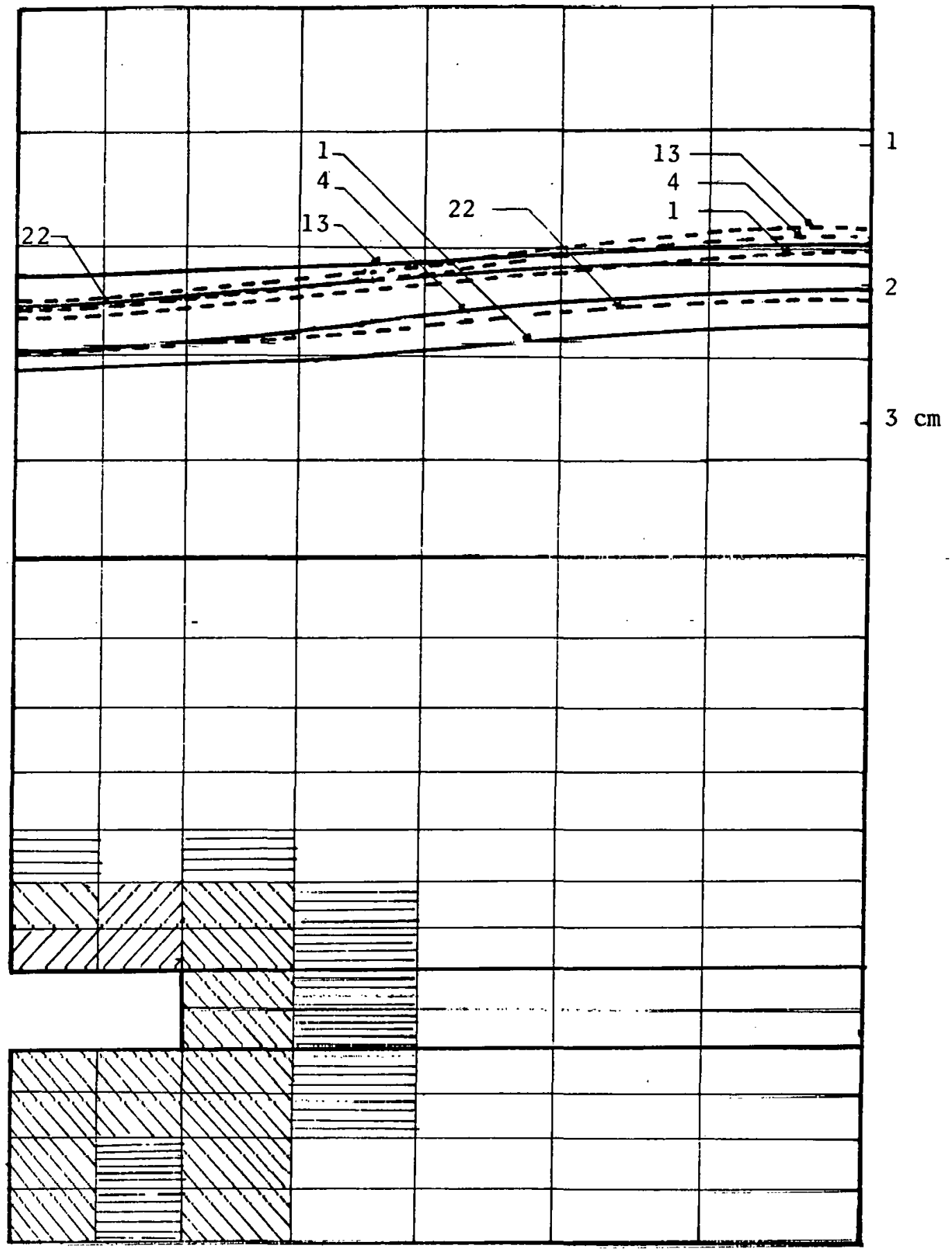

DDA $\mathrm{t}=.4$ month

$\angle \partial+\mathrm{W} \mathrm{t}=1$ month

$Z 7 D+\Delta V+\rightleftharpoons \mathrm{t}=19$ month
- Elastic Analysis

Elastoplastic Analysis

Figure 4.13 Surface Settlement History and Progression of Plastic Zones, Centralia Coal Gasification (Hatched area implies yielded element.) 
CHAPTER V

CONCLUSIONS

\subsection{Preliminary Remarks}

Governing equations and finite element formulations for the quasi-static infinitesimal theories of thermoelastic and thermoelastoplastic consolidation have been presented in this dissertation. Since there are no available solutions for such complex problems, the developed finite element codes have been validated against limiting cases available in the literature. The application of these investigations to underground coal gasification problems is also revealed.

\subsection{Concluding Remarks}

The major contributions of this dissertation are as

\section{follows :}

1. Using the theory of mixtures $[23,15]$ and the isothermal consolidation theory [48], the field equations for the quasi-static infinitesimal theory of thermoelastic consolidation are developed.

2. A variational principle equivalent to the field equations, initial and boundary conditions is developed using the approach in Refs. $[50,51]$. Extended variational principles 
as well as some specializations are also presented.

3. Finite element formulations based on the variational principle are presented. Two isoparametric elements are suggested in the spatial discretization.

4. The formulation is extended in order to account for plasticity effects. The rock medium is modeled as an elasticwork hardening material with an associated flow rule. Classical and recent yield criteria are included in the formulations.

5. The developed finite element codes are checked by solving available analytical and numerical solutions for isothermal consolidation, heat conduction, and elastoplastic analysis. Good agreements are observed between the results from developed codes and the available solutions.

6. Numerical efficiencies of the temporal and spatial interpolation schemes are investigated for a one-dimensional thermoelastic consolidation problem. It is found that for $\alpha=.5$, the 8-8-8 element gives an oscillatory pressure response. However, by increasing $a$, the 8-8-8 and the 8-4-4 elements gave identical results. The pressure is more influenced by the sudden change in the time increment than the Lempesdlure. However, the error associated with a sudden change in $\Delta t$ dies out in a few steps. Spatial and temporal discretization schemes have a negligible effect on the surface settlement. 
7. The excessive settlements observed in the thermoelastoplastic problem illustrate the importance of modeling the soil as an elastoplastic material and the need for conducting parameter sensitivity studies.

\subsection{Applications}

The presented formulation is applicable to problems of saturated continuum subjected to both thermal and mechanical loads. The continuum can be modeled as an elastic material or as an elastoplastic material with an associated flow rule. Geotechnical examples utilizing this formulation include problems dealing with underground coal gasification, geothermal energy recovery, hydraulic fracturing and nuclear waste management. Additionally, responses associated with polymer or composite structures subjected to hygrothermal loading can also be studied. 
5.4 Research Recommendations

The following research is recommended as a sequel to this study:

1. Inclusion of the coupling between heat and mass flow.

2. Inclusion of the effects of fluid compressibility.

3. Development and use of temperature dependent yield functions and material properties for the geotechuicd materials.

4. Consideration of effects of nonlinearity and anisotropy

5. Extension to thermo-visco-elasto-plastic consolidation formulations.

6. Consideration of temperature and stress dependent permeabilities, conductivites and thermal expansion cuefficients.

7. Incurporation of the effects on joint/fracture systells and bi-material interfaces. 
LIST OF REFERENCES

1. Basu, A.K., "New Light on the Nayak Alpha Technique," Letter to the Editor, Int. J. Num. Meth. Eng., 6, 152, 1973.

2. Biot, M.A., "General Theory of Three-Dimensional Consolidation," Journal of Applied Physics, 12, 155-164, 1941.

3. Biot, M.A., "Consolidation settlement Under a Rectangular Load Distribution," Journal of Applied Physics, 12 , $426-430$, 1941 .

4. Biot, M.A., "Theory of Deformation of a Porous Viscoelastic Anisotropic Solid," Journal of Applied Physics, $27,459-467,1956$.

5. Biot, M.A., "Thermoelasticity and Irreversible ThermoThermodynamics," Journal of Applied Physics, 27, 240$253,1956$.

6. Biot, M.A. and Willis, D.G., "The Elastic Coefficients of the Theory of Consolidation," Journal of Applied Physics, 24, 594-601, 1957 .

7. Boley, B.A. and Weiner, J.H., Theory of Thermal stresses, John Wiley \& Sons, Inc., 1960.

8. Booker, J.R. and Small, J.C., "An Investigation of the Stability of Numerical Solutions of Biot's Equations of Consolidation," Int. J. Solids Struct., 11, 907-917, 1975 .

9. Carslaw, H.S. and Jaeger, J.C., Conduction of Heat in Solids, D.R. Hillman and Sons Ltd., Great Britain, 1959.

10. Carter, J.P., Small, J.C. and Booker, J.R., "A Theory of Finite Elastic Consolidation," Int. J. Solids Struct., $13,467-478,1977$.

11. Carter, J.P., Booker, J.R. and Small, ..C.., "The Analysis of Finite Elasto-Plastic Consolidation," Int. J. Num. Meth. Geomechanics, 3, 107-129, 1979. 
12. Chen, W.F., Limit Analysis and Soil Plasticity, Elsevier Scientific Publishing Co., Amsterdam, Oxford and New York, 1975.

13. Chen, W.F., Plasticity in Reinforced Concrete, McGrawHill Book Company, 1982.

14: Christian, J.T. and Boehmer, J.W., "Plain Strain Consolidation by Finite Element Methods," ASCE, J. Soil Mech. and Foundation Div., 96, SMU, 1435-1457, 1970.

15. Crochet, M.J. and Naghdi, P.M, "On Constitutive Equations for Flow of Fluid Through an Elastic Solid," Int. J. Engng. Sci., 4, 383-401, 1966.

16. Desai, C.S. and Abel, J.F., Introduction to the Finite Element Method, A Numeriral Methoid for Enginegring hnalys1s, Vàn Nostrand Reinhold Compañy, 1972.

17. Desai, C.S., "Flow Through Porous Media," Numericel Methods in Geotechnical Engineering, Ed., C.S. Desái and J.T. Christian, 458-505, McGraw-Hill, Inc., 1977.

18. DiMaggio, F.L. and Sandler, I.S., "Material Models for Granular Soils," J. Eng. Mechanics Div., ASCE, 97, 936$950,1971$.

19. Drucker, C.D., Gibson, R.E. and Henkel, D.J., "Soil Mechanics and Work-Hardening Theories of Plasticity," Trans. ASCE, 122, 338-346, 1957.

20. Fung, Y.C., Foundations of Solid Mechanics, PrentireHall, Inc., 1965 .

21. Ghaboussi, J. and Wilson, E.L., "Flow of Compressible Fluid in Porous Elastic Media," Int. J. Num. Meth. Eng. Vol. 1, No. 3, 419-442, 1972 .

22. Ghaboussi, $j$, and Momer, H., "Plasticity Model for Cyclic Behavior of Sands," in Numerical Methods in Geomechanics, Vol. l, ed. W. Wittke, A.A. Blakema Rotterdam, 1979 .

23. Green, A.E. and Naghdi, P.M., "A Dynamical Theory of Interacting Continuua," Int. J. Engng. Sci., Vol. 3, 231-241, 1965 .

24. Gurtin, M.E., "Variational Principles for Linear Initial Value Problems," Quart. J. Appl. Math., 22, 252-756, 1964. 
25. Hwang, C.T., Morgenstern, N.R, and Murray, D.W., "On Solution of Plane Strain Consolidation Problems by Finite Element Methods," Canadian Geotech. J., Vol. 8, No. 1, 109-118, 1971 .

26. Kachanov, L.M., Fundamentals of the Theory of Plasticity, $M / R$ Publishers, Moscow English translation, 1974 .

27. Kammash, T.B., Murch, S.A. and Naghdi, P.M., "The Elastic-Plastic Cylinder Subjected to Radially Distributed Heat Source, Lateral Pressure and Axial Force with Application to Nuclear Reactor Fuel Elements," J. Mech. Phys. Solids, 8, 1-25, 1960 .

28. Krause, G., "Finite Element Schemes for Porous Elastic Media," J. Engrg. Mech. Div., ASCE, 104, 605-620, 1978.

29. Kreig, R.D. and Kreig, D.B., "Accuracies of Numerical Solution Methods for the Elastic-Perfectly Plastic Model," J. Pressure Vessel Technology, Trans. ASME, $99,510-515,1977$.

30. Lee, C.H. and Kobayashi, S., "Elastoplastic Analysis of Plane-Strain and Axisymmetric Flat Punch Identation by the Finite-Element Method," Int. J. Mech. Sci., 12, 349370,1970 .

31. Mendelson, A., Plasticity Theory and Application, The Macmillan Company, 1968.

32. Marcal, P.V., "A Stiffness Method for Elastic-Plástic Problems," Int. J. Mech. Sci., 7, 229-238, 1965.

33. Marcal, P.V. and King, I.P., "Elastic-Plastic Analysis of Two-Dimensional stress systems by the Finite Element Method," Int. J. Mech. Sci., 9, 143-155, 1967.

34. Mikhlin, S.G., The Problem of the Minimum of a Quadratic Functional, Holden-Day, 1965 .

35. Nadghi, P.M., Stress-Strain Relations in Plasticity and Thermoplasticity, Plasticity Proc. of the 2nd Symposium of Naval Structural Mechanics, Pergamon Press, 121-169, 1960 .

36. Nayak, G.C. and Zienkiewicz,O.C., "Convenient Form of Stress Invariants for Plasticity," J. Struct. Div., ASCE, 98, 949-954, 1971.

37. Nayak, G.C. and Zienkiewicz,O.C., "Elasto-Plastic Stress Analysis: A Generalization of Various Constitutive 
Relations Including strain Softening," Int. J. Num. Meth. Eng., 5, 113-135, 1972 .

38. Nayak, G.C. and Zienkiewicz, O.C., "Note on the 'Alpha' Constant Stiffness Method for Analysis of Nonlinear Problems," Int. J. Num. Meth. Eng., 4, 579-582, 1972.

39. Nickel, R.E. and Sackman, J.L., "Variational Principles for Linear Coupled Thermoelasticity," Quart. J. Appl. Math., 26, 11-26, 1968 .

40. Owen, D.R.J. and Hinton, E., Finite Elements in Plasticity Theory and Practice, Pineridge Press Limited, Swansee, U.K., 1980 .

41. Polubarinova-Kochina, P. Ya., The Theory of Ground Water Movement, princeton University Press, Princeton, Néw Jersey, 1962 .

42. Prager, W., "Non-Isothermal Plastic Deformation," Koninklijke Nederland Akademie Van Wetenschappen Te Amsterdam Proceedings of the section of Sciences, Ses. B, 61, 1958 .

43. Prevost, J.H., "Mechanics of Continuous Porous Media," Int. J. Engng. Sci., 18, 787-800, 1980 .

44. Prevost, J.H., "Consolidation of Anelastic Porous Media," J. Eng. Mechanics Div., ASCE, 107, 169-186, 1981.

45. Rice, J.R. and Tracy, D.M., "Computational Fracture Mechanics," in Numerical and Computer Methods in Structural Mechanics, ed. S.J. Fenver, Academic Press, New York, 585-623, 1973.

46. Roscoe, K.H., Schofield, A.N. and Thurairogah, ;' "Yielding of clays in states wetter than Critical," Geotechnique, 13,711-740, 1963.

47. Roscoe, K.H. and Poorooshasb, H.B., "A Theoretical and Experimental study of strains in Triaxial rompression Tests on Normally Consolidated Clays," Geotechnique, $13,12-38,1963$.

48. Sandhu, K.S., "Fluid Flow in Saturated Porous Elastic Media," Ph.D. Thesis, University of California at Berkeley, Berkeley, California, 1968.

49. Sandhu, R.S. and Wilson, E.I., "Finj.te Eleınent Analysis of Seepage in Elastic lledia," ASCE, J. Eng. Mech. Div." $95,641-652,1969$. 
50. Sandhu, R.S. and Pister, K., "A Variational Principle for Boundary Value and Initial Value Problems," Int. J. Solids Struc., 7, 639-654, 1971.

51. Sandhu, R.S., "Variational Principles for Soil Consolidation," National Science Foundation Grant GK-35900, The Ohio State University, Columbus, Ohio, 1975.

52. Sandhu, R.S. and Salaam, U., "Variational Formulation of Linear Problems with Nonhomogeneous Boundary Conditions and Internal Discontinuities," Computer Methods in Applied Mech. and Eng., 7, 75-91, 1975.

53. Sandhu, R.S., "Finite Element Analysis of Soil Consolidation," National Science Foundation Grant 72-04110-A, Geotechnical Engineering Report No. 6, The Ohio State University, Columbus, Ohio, 1976.

54. Scheidegger, A.E., The Physics of Flow Through Porous Media, MacMillan Co., New York, 1960 .

55. Schiffman, R.I., "A Thermoelastic Theory of Consolidation," Environmental and Geophysical Heat Transfer, 4, 78-84, Heat Transfer Div., Amer. Soc. of Mech. Engrs., New York, 1972 .

56. Schiffman, R.L. and Arya, S.K., "One-Dimensional Consolidation," Numerical Methods in Geotechnical Engineering, C.S. Desai and J.T. Christian, McGraw-Hill, Inc. $364-398,1977$.

57. Schofield, A.N. and Worth, P., Critical State Soil Mechanics, McGraw-Hill, New York, 1968 .

58. Schreyer, H.L., Kulak, R.F. and Kramer, J.M., "Accurate Numerical Solutions for Elastic-Plastic Models," ASME $\mathrm{J}$. of Pressure Vessel Technology, 101, 226-234, 1979.

59. Sharabi, M.N., "Finite Element Solutions of Nonlinear Coupled Flow-Stress Problems Based on Mixture Theory," Ph.D. Thesis, University of Texas at Austin, 1975.

60. Singh, R.D., "Mechanical Characterization and Finite Element Analysis of Elastic-Plastic, Work-Hardening Soils," Ph.D. Thesis, The Ohio State University, 1972.

61. Siriwardane, H.J. and Desai, C.S., "Two Numerical Schemes for Nonlinear Consolidation," Int. J. Num. Meth. Eng., 17, 405-426, 1981 . 
62. Small, J.C., Booker, J.R. and Davis, E.H., "ElastoPlastic Consolidation of Soil," Int. J. Solids Struct., $12,431-448,1976$.

63. Smith, I.M. and Hobbs, R., "Biot Analysis of Consolidation Beneath Embankments," Geotechnique, Vol. 26, No. 1, $149-171,1976$.

64. Terzaghi, and R,B. Peck, Soil Mechanics in Engineering Practice, J. Wiley, 1948 .

65. Witherspoon, P.A., Tsang, J.,. Long, C.S., and Noorishad, $\mathrm{J}$., "New Approach to Problems of Fluid Flow in Fractured Rock Masses," 22nd U.S. Symposium on Rock Mechanics, 120, MIT, 1981 .

66. Yamada, $Y$. and Yoshimura, N., "Plactio stroes-Strain Matrix and Its Application for the Solution of ElasticPlastic Problems by the Finite Element Method," Int. J. Mech. Sci., 10, 343-354, 1968.

67. Yokoo, Y., Yamagata, K., Nagaonka, H., "Finite Element Methods Applied to Biot's Consolidation Theory," Soils and Foundation, Vol. 11, No. 1, 29-46, 1971.

68. Yooko, Y., Yamagata, K., Nagaonka, H., "Variational Principles for Consolidation," Soils and Foundation, Vol. 11, No. 4, 25-35, 1971 .

69. Yooko, Y., Yamagota, K., Nagaonka, H., "Finite Element Analysis of Consolidation Following Undrained Deformation," Solls and Foundation, Vul. 11, No. 4, 37-58, 1971.

70. Zienkiewicz, O.C., Valliappan, S. and King, I.P., "Elasto-Plastic Solutions of Engineering Problems, Initial Stress Finite Element Approach," Int. J. Num. Meth. Eng., 1, 75-100, 1969 . 


\section{APPENDIX A}

\section{Classical Yield Criteria and Alternative Forms}

\section{A. 1) General}

The yield criterion determines the stress level at which plastic deformations begin and can be expressed as

$$
f\left(\sigma_{i j}\right)=K(k)
$$

or equivalently

$$
F\left(\sigma_{i j}, k\right)=0
$$

where $f$ is an invariant function of the state of stress, $\mathrm{K}$ is a material parameter to be determined experimentally and $\mathrm{k}$ is a hardening parameter. Among the classical yield criteria we cite Tresca, Von Mises, Mohr-Coulomb and Drucker-Prager. In the following a compact summary of these criteria is presented. The reader is referred to Refs $[12,13]$ for additional information. An illustration of these yield criteria is given in Figs A.1 and A.2.

\section{A. 2) Tresca Yield Criterion}

The Tresca Yield Criterion states that yielding of a material begins when the maximum shearing stress at a point reaches the value of the maximum shear stress occurring under simple tension. For $\sigma_{1} \geq \sigma_{2} \geq \sigma_{3}$, Tresca's criterion 
can be expressed as $1 / 2\left(\sigma_{1}-\sigma_{3}\right)=1 / 2 \sigma_{0}$

(A. 3)

where $\sigma_{0}$ is the uniaxial yield stress in simple tension. It should be noted that the yield stress in pure shear, $k$, is given by

$$
\mathrm{K}=1 / 2 \sigma_{0}
$$

\section{A. 3) Von Mises Yield Criterion}

The Von Mises Yield Criterion states that yielding of the material begins when the distortional energy equals the alstortional energy at yield in simple tenston, i.e.

$$
\frac{1}{2 \mathrm{G}} \mathrm{J}_{2}=\frac{1}{2 \mathrm{G}} \frac{1}{3} \sigma_{0}^{2}
$$

where $J_{2}$ is the second invariant of the deviatoric stress tensor defined by

$$
J_{2}=1 / 2 s_{i j} \cdot s_{i j}
$$

It should be noted that the yield stress in pure shear, $K$, is given by

$$
\mathrm{K}=\frac{\mathrm{o}_{\mathrm{O}}}{\sqrt{3}} .
$$

\section{A. 4) Mohr-Coulomb Yield Criterion}

The Mohr-Coulomb Yield Criterion states that yielding of the material begins when the maximum shear stress at a point is given by

$$
\tau=c-\sigma_{n} \tan \phi
$$


where $\tau$ is the magnitude of the shearing stress, $\sigma_{n}$ is the normal stress, $C$ is the cohesion and $\phi$ is the angle of internal friction. For $\sigma_{1} \geq \sigma_{2} \geq \sigma_{3}$, Eq (A.8) can be rewritten as

$$
1 / 2\left(\sigma_{1}-\sigma_{3}\right)=\mathrm{C} \cos \phi-1 / 2\left(\sigma_{1}+\sigma_{3}\right) \sin \phi .
$$

For the case of a frictionless material, $\phi=0$, the MohrCoulomb yield criterion reduces to the Tresca yield criterion with $\mathrm{C}=\mathrm{K}$.

\section{A.5) Drucker-Prager Yield Criterion}

The Drucker-Prager Yield Criterion is an approximation to the Mohr-Coulomb yield criterion and a modification of the Von Mises yield criterion. The influence of the hydrostatic stress component on yielding is introduced by inclusion of an additional term in the Von Mises Yield Criterion to give

$$
\alpha^{\prime} I_{1}+\sqrt{J_{2}}=I^{\prime}
$$

where $I_{1}$ is the first stress invariant, i.e.

$$
I_{1}=\sigma_{i i}
$$

$\alpha^{\prime}$ and $K^{\prime}$ are material constants that can be related to $C$ and $\phi$ by

$$
\alpha^{\prime}=\frac{2 \sin \phi}{\sqrt{3}(3-\sin \phi)}, K^{\prime}=\frac{6 c \cos \phi}{\sqrt{3}(3-\sin \phi)}
$$


In this case the Drucker-Prager circle coincides with the outer apices of the Mohr-Coulomb hexagon. Coincidence with the inner apices of the Mohr-Coulomb hexagon is provided by

$$
\alpha^{\prime}=\frac{2 \sin \phi}{\sqrt{3}(3+\sin \phi)}, K^{\prime}=\frac{6 C \cos \phi}{\sqrt{3}(3+\sin \phi)}
$$

A.6) Alternative Form of the Yield Criterion for Numerical Computation

This formulation is due to Nayak and Zienkiewicz [36], and its main advantage is that it permits the computer coding of the yield function and the flow rule in a general form and requires only the specification of three constants for any individual criterion.

The principal deviatoric stresses $S_{i}$ are given as the roots of the cubic equation

$$
S^{3}-J_{2} S^{2}-J_{3}=0 \text {. }
$$

Substituting $S=r \sin \theta$ in Eq (A.14) gives

$$
\sin ^{3} \theta-\frac{J_{2}}{r^{2}} \sin \theta-\frac{J_{3}}{r^{3}}=0
$$

Noting the triqonometric identity

$$
\sin ^{3} \theta-\frac{3}{4} \sin \theta+\frac{1}{4} \sin 3 \theta=0
$$

and comparing (A.15) and (A.16) gives

$$
r=2 \sqrt{J_{2}} / \sqrt{3}
$$




$$
\sin 3 \theta=-\frac{4 J_{3}}{r^{3}}=-\frac{3 \sqrt{3}}{2} \frac{J_{3}}{J_{2} \sqrt{J_{2}}}
$$

The first root of Eq (A.18) with $3 \theta$ in the range $\pm \frac{\pi}{2}$ was chosen as an alternative to $J_{3}$. Noting the cyclic nature of $\sin (3 \theta+2 n \pi)$, the principal stresses are

$$
\left\{\begin{array}{l}
\sigma_{1} \\
\sigma_{2} \\
\sigma_{3}
\end{array}\right\}=\frac{2 \sqrt{\mathrm{J}_{2}}}{\sqrt{3}}\left\{\begin{array}{ll}
\sin \left(\theta+\frac{2 \pi}{3}\right) \\
\sin \theta \\
\sin \left(\theta+\frac{4 \pi}{3}\right)
\end{array}\right\}+\frac{I_{1}}{3}\left\{\begin{array}{l}
1 \\
1 \\
1
\end{array}\right\}
$$

with $\sigma_{1}>\sigma_{2}>\sigma_{3}$ and $-\frac{\pi}{6} \leq \theta \leq \frac{\pi}{6}$.

\section{A.6.1) Yield Criteria}

The four yield criteria considered in sections (A.2)

through (A.5) can be rewritten in terms of

$I_{1}, J_{2}, \theta$ as follows:

\section{(i) Tresca Yield Criterion:}

Substituting for $\sigma_{1}$ and $\sigma_{3}$ from $\mathrm{Eq} \mathrm{(A.19)} \mathrm{into} \mathrm{Eq} \mathrm{(A.3)}$ gives

$$
2 \sqrt{J_{2}} \cos \theta=\sigma_{0}
$$

\section{(ii) Von Mises Yield Criterion:}

There is no change from the conventional form since the yield function depends on $\mathrm{J}_{2}$ only,

$$
\sqrt{3} \sqrt{J_{2}}=\sigma_{0}
$$

(iii) Mohr-Coulomb Yield Criterion:

Substituting for $\sigma_{1}$ and $\sigma_{3}$ from Eq (A.19) into Eq (A.9) gives $-95-$ 


$$
\begin{aligned}
\frac{1}{3} I_{1} \sin \phi+\sqrt{J_{2}}(\cos \theta & \left.-\frac{1}{\sqrt{3}} \sin \theta \sin \phi\right) \\
& =c \cos \phi
\end{aligned}
$$

(iv) Drucker-Prager Yield Criterion:

There is no change from the conventional form, i.e.,

$$
\alpha^{\prime} I_{1}+\sqrt{J_{2}}=K^{\prime}
$$

Fig (A.3) illustrates the intersection of these four yield criteria with the $\Pi-p l a n e$.

\section{A.6.2) Flow Rule}

The flow rule specifies the direction of the plastic. strain increment vector. The associated flow rule means that the plastic strain increment vector is normal to the yield surface at the current state of stress. Thus for purpose of elostoplastic analysis with the associated flow rule, it is necessary to evaluate the normal vector, or the derivative of the yield function with respect to stresses.

$$
\underset{\sim}{\mathrm{a}}=\frac{\partial \mathrm{F}}{\partial \underset{\sim}{\sigma}}=\frac{\partial F}{\partial \mathrm{I}_{1}} \frac{\partial \mathrm{I}_{1}}{\partial \underset{\sim}{\sigma}}+\frac{\partial \mathrm{F}}{\partial \mathcal{J}_{2}} \frac{\partial \sqrt{ } \mathrm{J}_{2}}{\partial \underset{\sim}{\sigma}}+\frac{\partial F}{\partial \theta} \frac{\partial \theta}{\partial \underset{\sim}{\sigma}}
$$

whe re

$$
\stackrel{\sigma}{\sigma}^{\mathrm{T}}=\left\{\sigma_{\mathrm{xx}}, \sigma_{\mathrm{yy}}, \sigma_{\mathrm{zz}}, \sigma_{\mathrm{xy}}, \sigma_{\mathrm{xz}}, \sigma_{\mathrm{yz}}\right\}
$$

Noting from Eq (A.18) that

$$
\frac{\partial \theta}{\partial \underset{\sim}{\sigma}}=\frac{-\sqrt{3}}{2 \cos 3 \theta}\left[\frac{1}{J_{2} \sqrt{J_{2}}} \frac{\partial J_{3}}{\partial \sigma}-\frac{\partial J_{3}}{\partial J_{2}} \frac{\partial \sqrt{ } J_{2}}{\partial \underset{\sim}{\sigma}}\right]
$$


Eq (A.24) can be rewritten as

$$
\underset{\sim}{a}=c_{1} \underset{\sim}{a_{1}}+c_{2} \underset{\sim}{a_{2}}+c_{3} \underset{\sim}{a_{3}}
$$

where

$$
\begin{aligned}
& \underset{\sim}{a_{1}^{T}}=\frac{\partial I_{1}}{\partial \underset{\sim}{\sigma}}=\{1,1,1,0,0,0\} \\
& \underset{\sim}{\mathrm{a}_{2}^{\mathrm{T}}}=\frac{\partial \sqrt{\mathrm{J}_{2}}}{\partial \underset{\sim}{\sigma}}=\frac{1}{2 \sqrt{J_{2}}}\left\{\mathrm{~s}_{\mathrm{xx}}, \mathrm{s}_{\mathrm{yy}}, \mathrm{s}_{z \mathrm{z}}, \mathrm{s}_{\mathrm{xy}}, \mathrm{s}_{\mathrm{xz}}, \mathrm{s}_{\mathrm{yz}}\right\} \\
& \underset{\sim}{a} \underset{3}{T}=\frac{\partial J_{3}}{\partial \underset{\sim}{\sigma}}=\left\{\left(S_{Y Y} S_{z z}-S_{Y z}^{2}+\frac{J_{2}}{3}\right),\left(S_{X x} S_{z z}-S_{x}^{2}+\frac{J_{2}}{3}\right),\right. \\
& \left(s_{x x} s_{y y}-s_{x y}^{2}+\frac{J_{2}}{3}\right),\left(s_{x z} s_{x y}-s_{x x} s_{y z}\right), \\
& \left.\left(s_{x y} s_{y z}-s_{y y} s_{x}\right),\left(s_{y z} s_{x}-s_{z z} s_{x y}\right)\right\}
\end{aligned}
$$

(A. 28)

and

$$
\begin{aligned}
& c_{1}=\frac{\partial F}{\partial I_{1}} \\
& c_{2}=\frac{\partial F}{\partial \sqrt{J_{2}}} \frac{\tan 3 \theta}{J_{2}} \frac{\partial F}{\partial \theta} \\
& c_{3}=\frac{-\sqrt{3}}{2 \cos 3 \theta} \frac{1}{J_{2} \sqrt{J_{2}}} \frac{\partial F}{\partial \theta}
\end{aligned}
$$

Only the constants $c_{1}, c_{2}$ and $c_{3}$ are then necessary to define the yield surface. Thus simplicity of programming can be achieved as only these three constants have to be varied between one yield surface and another. These three constants are given in the following table for the four yield criteria mentioned earlier.

$-97-$ 
Table A-I. Definition of C Constants

\begin{tabular}{|c|c|c|c|}
\hline Yield Criteria & $c_{1}$ & $c_{2}$ & $c_{3}$ \\
\hline Tresca & 0 & $2 \cos \theta(1+\tan \theta \tan 3 \theta)$ & $\frac{\sqrt{3} \sin \theta}{\sqrt{3} \cos 3 \theta}$ \\
\hline Von Mises & 0 & $\sqrt{3}$ & 0 \\
\hline Mohr-Coulomb & $\frac{1}{3} \sin \theta$ & $\begin{aligned} & \cos \theta[(1+\tan \theta \tan 3 \theta) \\
+ & \left.\frac{\sin \theta}{\sqrt{3}}(\tan 3 \theta \tan \theta)\right]\end{aligned}$ & $\frac{(\sqrt{3} \sin \theta+\cos \theta \sin \theta}{2 J_{2} \cos 3 \theta}$ \\
\hline Drucker-Prager & $\alpha^{\prime}$ & 1 & 0 \\
\hline
\end{tabular}




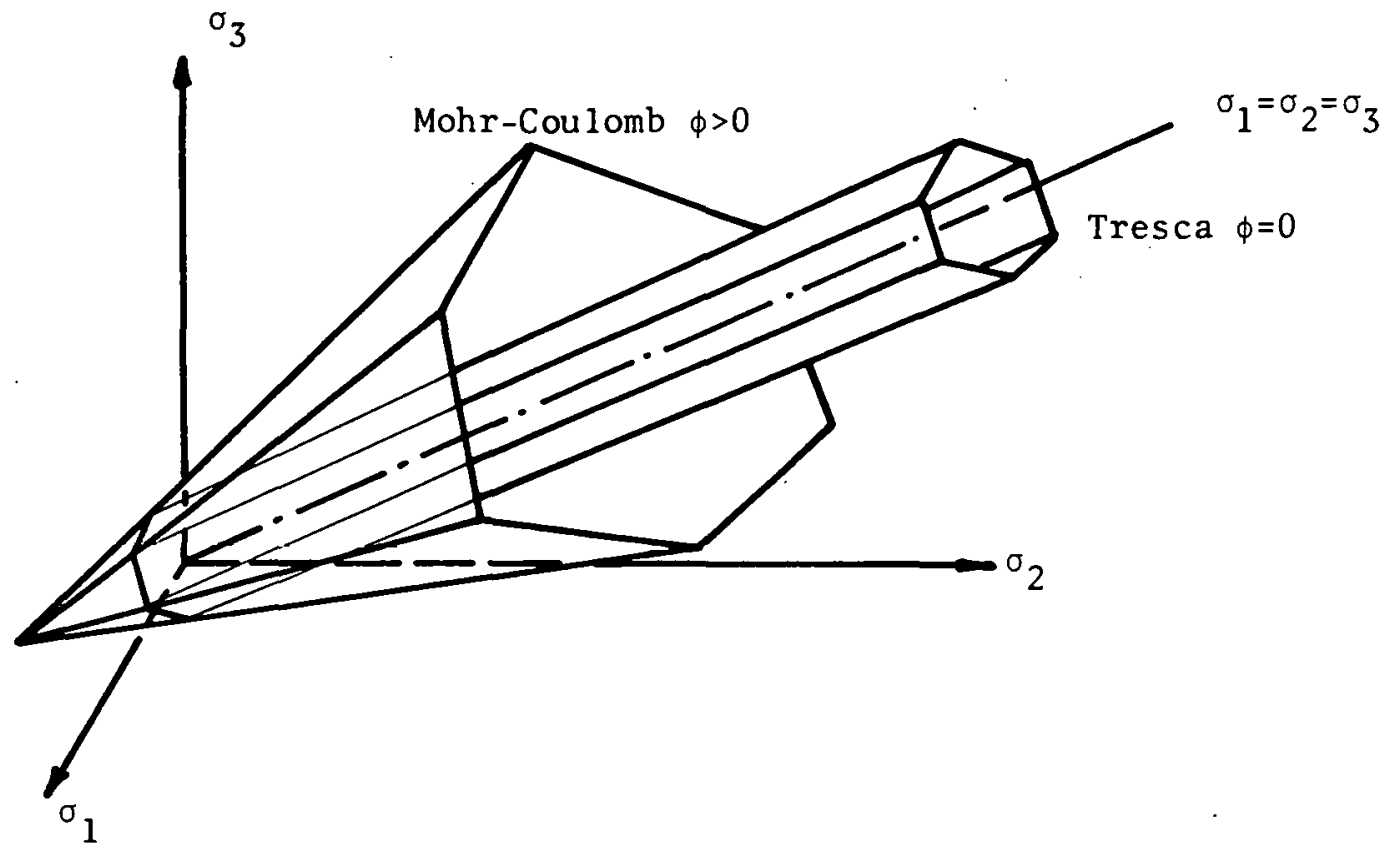

Figure (A.1): Mohr-Coulomb and Tresca Yield Criteria

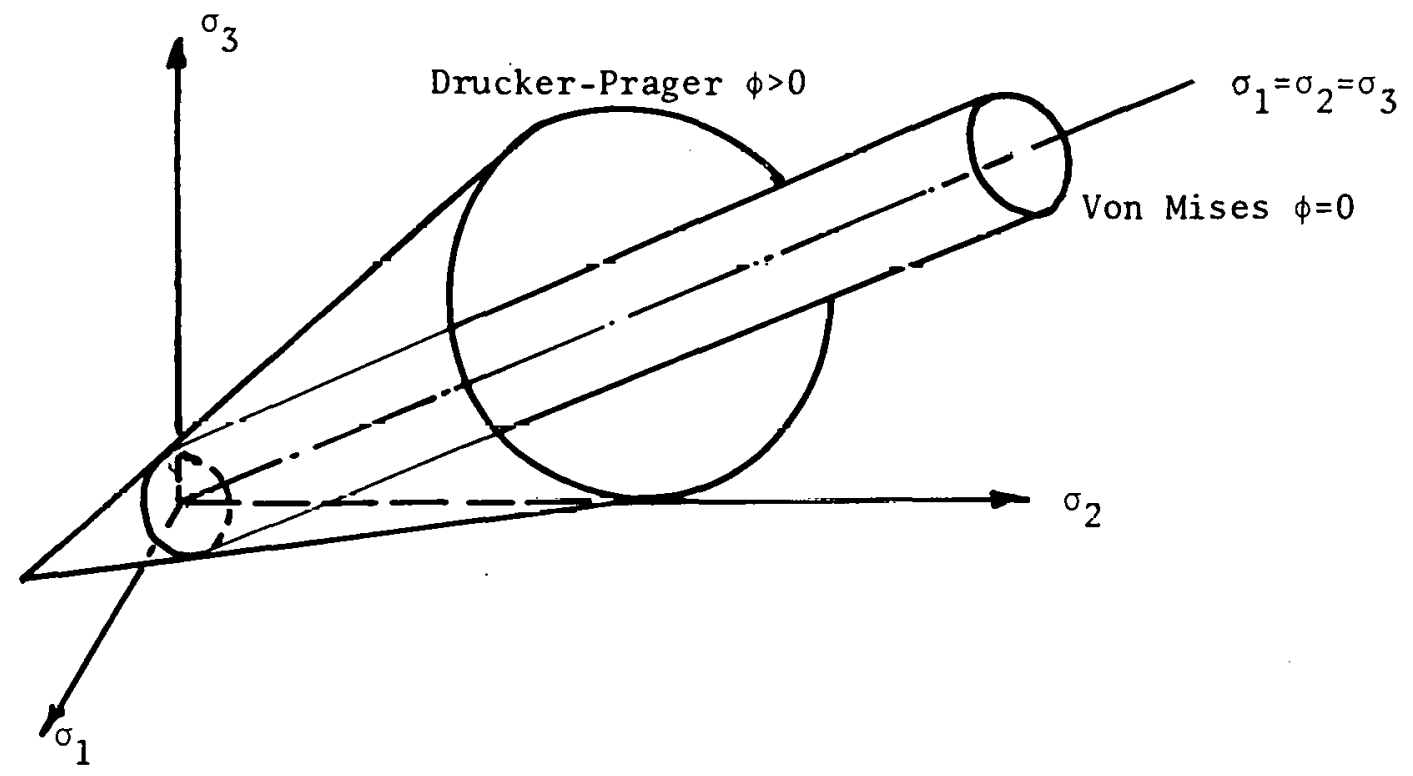

Figure (A.2): Drucker-Prager and Von Mises Yield Criteria 


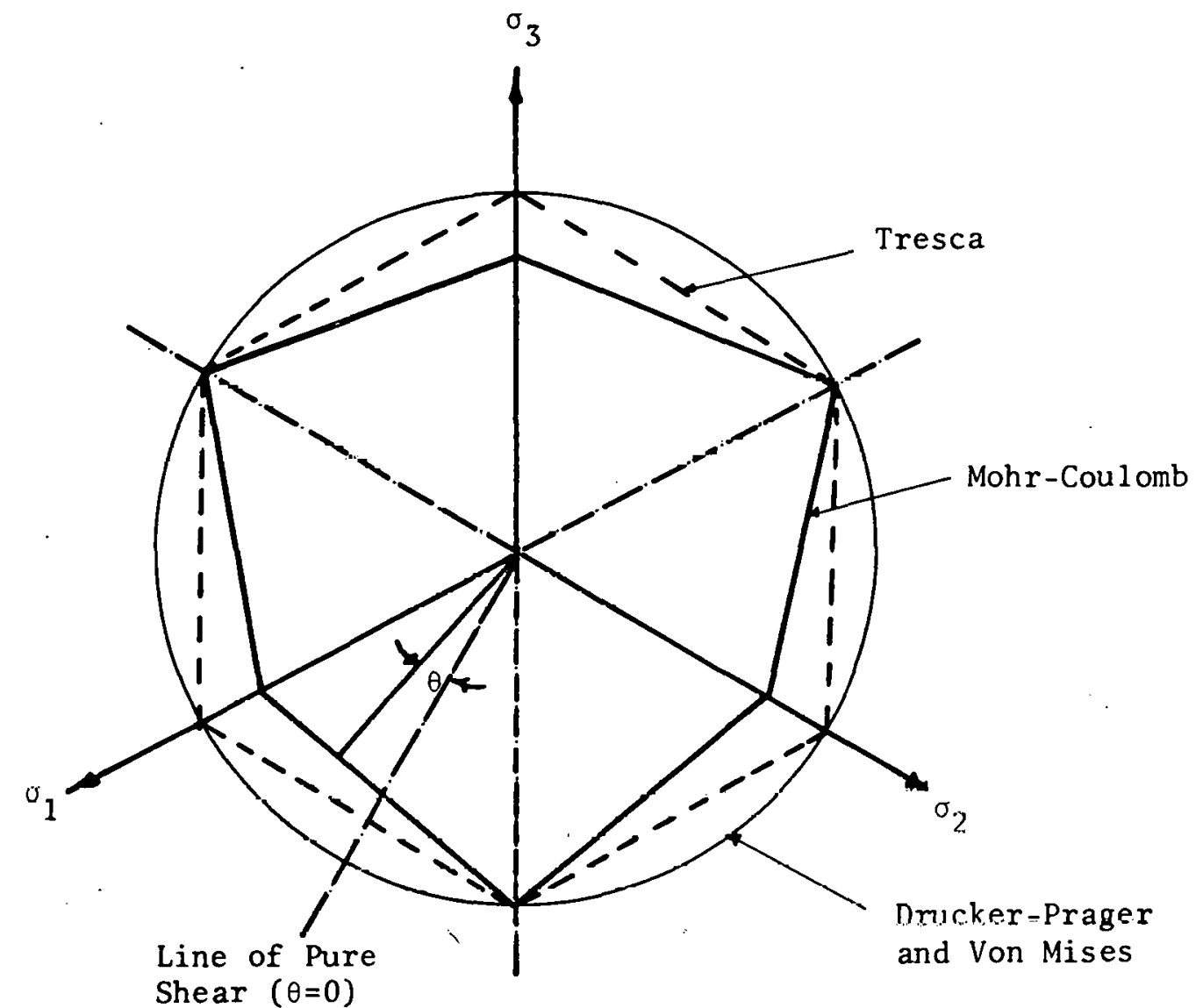

Figure (A.3): $\pi$-plane Representation of Tresca, Von Mises Mohr-Coulomb and Drucker-Prager Yield Criteria 
APPENDIX B

Yield Surface with an Expanding Cap

\section{B. 1) General}

The volumetric plastic strain increment, using the Drucker-Prager yield criterion Eq (A.10) with the associated flow rule Eq (3.19), is given by

$$
d e_{i i}^{p}=d \lambda \frac{\partial f}{\partial \sigma}=d \lambda \alpha^{\prime}
$$

Since $\alpha^{\prime} \geq 0$, the volumetric plastic strain is always nonnegative. This creates what is known as the dilatancy, i.e., an increase in plastic volume change under hydrostatic compression. To control this dilatancy, a volume dependent cap surface under hydrostatic compression is introduced. Drucker [19] was the first to introduce a spherical cap to control dilatancy of soils. Since then several plasticity models using the critical state concept have been developed, Ref [57] and a specific Cam clay model was suggested by Roscoe, et al. [46], for normallyconsolidated clays. Recently, the cap model has been modified by DiMaggio and Sandler [18], Singh [60] and Ghaboussi, et al. [22]. In Section (B-2) the cap modifications introduced by singh [66] are discussed. 


\section{B.2) Modified Cap Model [60]}

Fig (B.I) illustrates the model which consists of two parts:

a) Failure envelope in the form of a perfectly plastic Drucker-Prager line.

b) Yield surface consists of infinitely many elliptic caps each possessing a certain plastic volumetric strain. Mathmatically, the model can be expressed as

$$
\begin{array}{ll}
\alpha^{\prime} I_{1}+\sqrt{J_{2}}=K^{\prime} & I_{1} \geq P_{O} \\
\left(\frac{I_{1}-P_{0}}{a}\right)^{2}+\frac{J_{2}}{b_{2}}=1 & P_{C} \leq I_{1} \leq P_{O}
\end{array}
$$

where $\mathrm{P}_{0} \leq 0$ is the cap center, $\mathrm{a}$ and $\mathrm{b}$ are the major and minor axis of the ellipse and related by

$$
\mathrm{a}=\mathrm{R} \mathrm{b}
$$

and $P_{C}$ is given by

$$
P_{c}=P_{0}-a
$$

\section{B.2.1) Cap Geometry:}

Given the location of a certain point $\left(I_{1}, \sqrt{J_{2}}\right)$ in the stress space, the cap center, $\mathrm{P}_{\mathrm{O}^{\prime}}$, can be determined by solving Eqs (B.2) and (B.3), i.e.,

$$
P_{0}=\frac{-B+\sqrt{B^{2}-4 A C}}{2 A}
$$


where $A=1-\alpha^{\prime 2} \mathrm{R}^{2}$

$$
\begin{aligned}
& B=2 \alpha^{\prime} R^{2} K^{\prime}-2 I_{1} \\
& C=I_{1}^{2}+R^{2} J_{2}-R^{2} K^{\prime}
\end{aligned}
$$

Thus the dimension of the cap can be obtained by

$$
\begin{aligned}
& \mathrm{b}=\mathrm{K}^{\prime}-\alpha^{\prime} \mathrm{P}_{0} \\
& \mathrm{a}=\mathrm{R} \mathrm{b}
\end{aligned}
$$

\section{B.2.2) Cap Hardening}

Following Roscoe, et al. [47], the plastic volumetric strain is

$$
\left.e_{i i}^{p}=-\frac{1}{2 \cdot 3}\left(\frac{\Lambda-\zeta}{1+e_{0}}\right) \text { Ln } \frac{-P_{c}}{-P_{0}}\right)
$$

where $e_{0}$ is the initial void ratios, $\Lambda$ and $\zeta$ are the slopes of the compression and rebound curves for an idealized soil, Fig (B.2).

The hardening function $h$ can be written as

$$
h=-\frac{\partial F}{\partial \sigma_{j}} \quad \frac{\partial F}{\partial e p}
$$

which is zero for the Drucker-Prager line and except for the cap region will be given by

$$
h=\frac{12 b^{3}}{G(I+\alpha R)}\left(I_{1}-P_{0}\right)\left[\left(I_{1}-P_{0}\right)\left\{b+\alpha\left(I_{1}-P_{0}\right)+\alpha R^{2}\left(J_{2}-2 b^{2}\right)\right\}\right.
$$


where

$$
G=\frac{1}{2 \cdot 3} \frac{\Lambda-\zeta}{1+e_{0}}
$$

\section{B.2.3 Flow Vector}

Using the approach of Nayak and Zienkiewicz [36], presented in Appendix A, the flow vector for the cap is

$$
\underset{\sim}{a}=c_{1} \frac{\partial I_{1}}{\partial \underset{\sim}{\sigma}}+c_{2} \frac{\partial \sqrt{J_{2}}}{\partial \underset{\sim}{\sigma}}+c_{3} \frac{\partial J_{3}}{\partial \underset{\sim}{\sigma}}
$$

where

$$
\begin{aligned}
& c_{1}=\frac{\partial F}{\partial I_{1}}=2 b^{2}\left(I_{1}-P_{0}\right) \\
& c_{2}=\frac{\partial F}{\partial \sqrt{J_{2}}}=2 R^{2} b^{2} \sqrt{J_{2}} \\
& c_{3}=\frac{\partial F}{\partial J_{3}}=0
\end{aligned}
$$

\section{B.2.4) Subsequent Yield Caps}

In plastic loading, both initial and subsequent stress states must satisfy the yield condition. This requires the knowledge of both initial and subsequent yield surfaces. . The initial yield surface is very easy to locate by knowing the stress state at the beginning of an increment as described in section (B.2.1). The subsequent yield cap can be obtained as follows, Ref.[12]. 
Differentiating Eq (B.12) gives

$$
\mathrm{dP}_{\mathrm{C}}=-1 / \mathrm{G} \mathrm{P}_{\mathrm{C}} \mathrm{d} \mathrm{e}_{\mathrm{i} i}^{\mathrm{p}}
$$

The point $\left(\mathrm{P}_{\mathrm{C}}+\mathrm{dP}_{\mathrm{C}}, 0\right)$ lies on the new cap and the approach presented in section (B.2.1) can be used to locate that cap. 


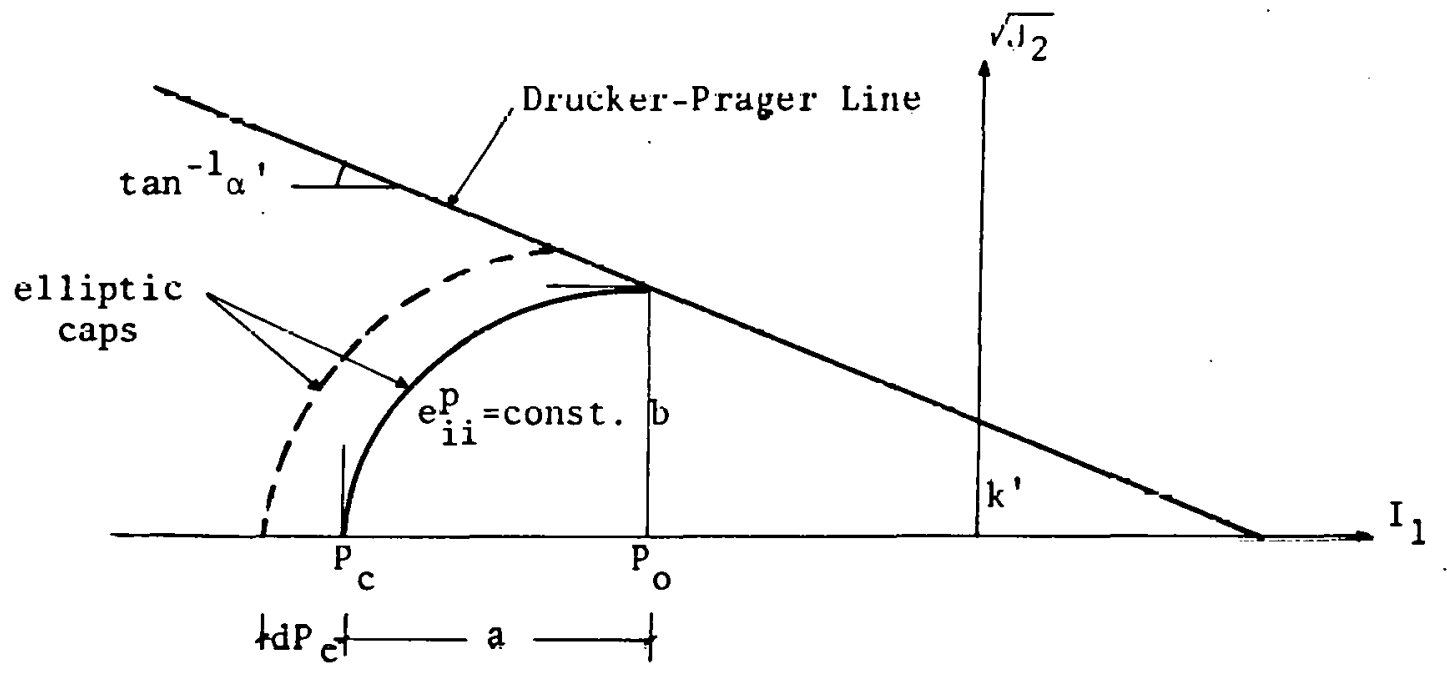

Figure (B.1): Elliptic Cap Mode1 


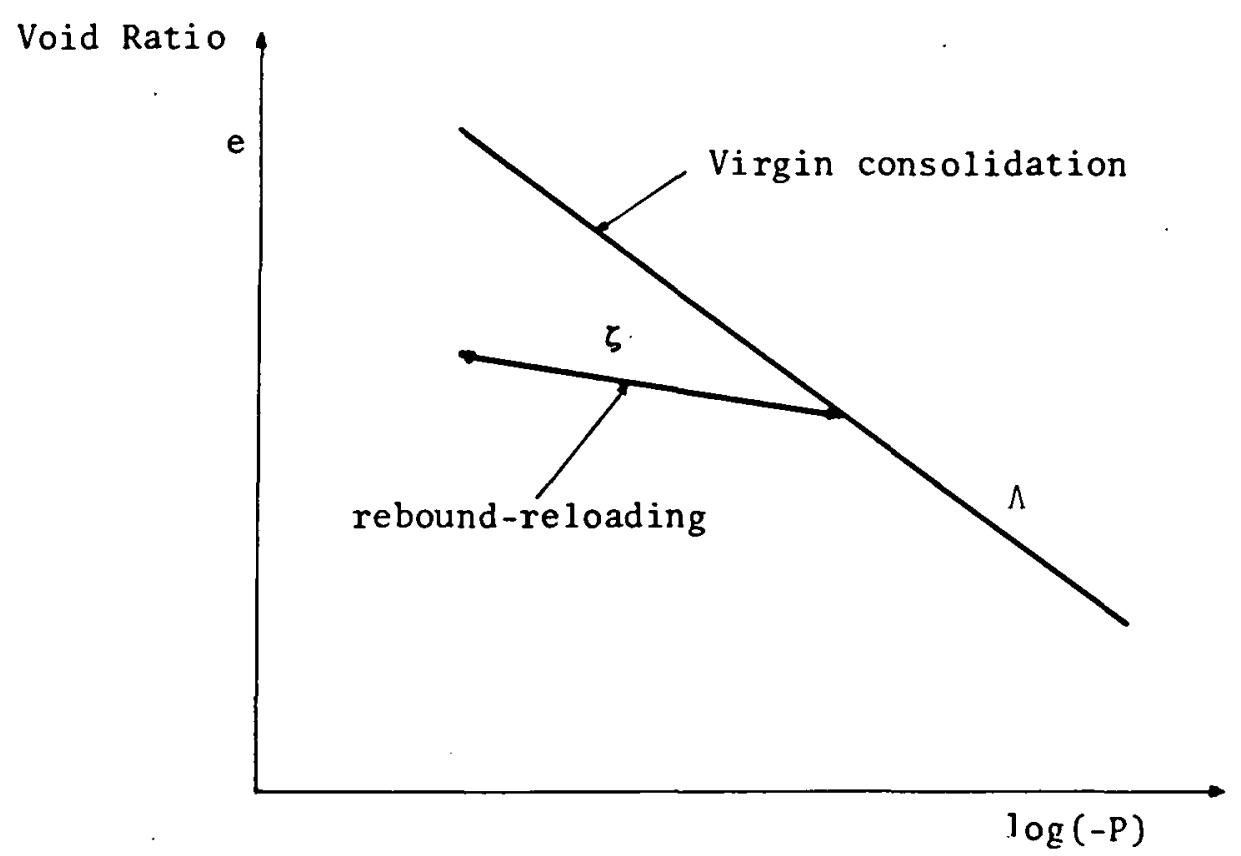

Figure (B.2): Response of Idealized Soil to Hydrostatic Stress 


\begin{abstract}
APPENDIX C
Incremental Stress Strain Relations in Plane strain

For plane strain, the general expressions for incremental stress strain relation, derived in Chapter ITT, have to be modified. Primarily the main alteration required is the deletion of the strain and stress components which vanish under the condition of plane strain. $\left(e_{z z}=e_{x z}=e_{y z}=0\right)$ Thus the effective stress vector will

$$
\stackrel{\sim}{\sigma}^{T}=\left\{\bar{\sigma}_{x x}, \bar{\sigma}_{y y}, \bar{\sigma}_{x y}, \bar{\sigma}_{z z}\right\}
$$
be

and the flow vector $\underset{\sim}{a}$ in Eq (A.24) will be

$$
a^{T}=\left\{\frac{\partial F}{\partial \bar{\sigma}_{x x}} ; \frac{\partial F}{\partial \bar{\sigma}_{Y Y}}, 2 \frac{\partial F}{\partial \bar{\sigma}_{x y}} ; \frac{\partial F}{\partial \bar{\sigma}} \underset{z z}{ }\right\}
$$

which corresponds to plastic strain increment given by

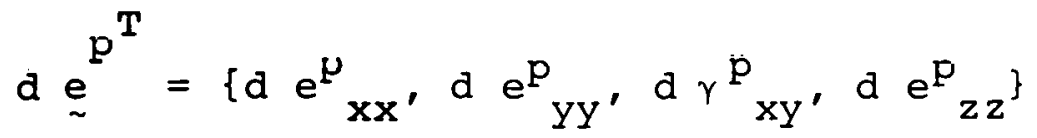

Eq (3.33) can be rewritten as

$$
d \underset{\sim}{\bar{\sigma}}=\left[D^{e p}\right] d \underset{\sim}{e}-{\underset{\sim}{\beta}}^{e p} d T
$$


where

$$
\begin{aligned}
& D_{i j k l}^{e p}=D_{i j k l}-D_{i j k l}^{p} \\
& D_{i j k l}=2 \mu \delta_{i k} \delta_{j l}+\lambda \delta_{k l} \delta_{i j} \\
& D_{i j k l}^{p}=\frac{D_{i j q r} \frac{\partial f}{\partial \bar{\sigma}_{s t}} \frac{\partial f}{\partial \bar{\sigma}_{S t}} D_{s t k l}}{h+\frac{\partial f}{\partial \bar{\sigma}_{m n}} \frac{\partial F}{\partial \bar{\sigma}_{u v}}} D_{\text {mnuv }}
\end{aligned}
$$

From Eq (A.24) $\frac{\partial f}{\partial \bar{\sigma}_{i j}}=\underset{\sim}{a}$

We let

$$
D_{i j k l} \frac{\partial f}{\partial \sigma_{k l}}=[D] \underset{\sim}{a}=\underset{\sim}{d}
$$

and obtain from Eq (C.7)

$$
\left[D^{\mathrm{P}}\right]=\frac{\stackrel{d}{\mathrm{~d}}{\stackrel{\sim}{a^{\mathrm{T}}}}^{\mathrm{T}}}{\mathrm{h}+{\underset{\sim}{\mathrm{a}}}^{\mathrm{T}} \underset{\sim}{\mathrm{d}}}
$$

We let

$$
\gamma=\mathrm{h}+{\underset{\sim}{\mathrm{a}}}^{\mathrm{T}} \underset{\sim}{\mathrm{d}}
$$

and rewrite $\mathrm{Eq}$ (C.5) in the form

$$
\left[D^{e P}\right]=[D]-\frac{1}{\gamma} \underset{\sim}{d} d_{\sim}^{T}
$$

We define $\beta_{i j}^{e p}$

$$
\beta_{i j}^{e p}=\beta_{i j}-\beta_{i j}^{p}
$$


where

$$
\begin{aligned}
& \beta_{i j}=\beta \delta_{i j}=(2 \mu+3 \lambda) \bar{\alpha} \delta_{i j} \\
& \beta_{i j}^{p}=\frac{D_{i j q r} \frac{\partial f}{\partial \bar{\sigma}_{q r}} \overline{\partial f}_{\text {d }}}{h+\frac{\partial f}{\partial \bar{\sigma}_{m n}} D_{m n u v} \frac{\partial f}{\partial \bar{\sigma}_{u v}}}
\end{aligned}
$$

Then ${\underset{\sim}{\beta}}^{p}$ can be written as

$$
\stackrel{\sim}{\beta}^{p}=\beta \frac{c_{1}}{\gamma} \underset{\sim}{d}
$$

where $c_{1}=\frac{\partial F}{\delta \pi_{i 1}}$ is defined in Table $(\Lambda .1)$ for different yield criteria.

The matrix [D] is defined by

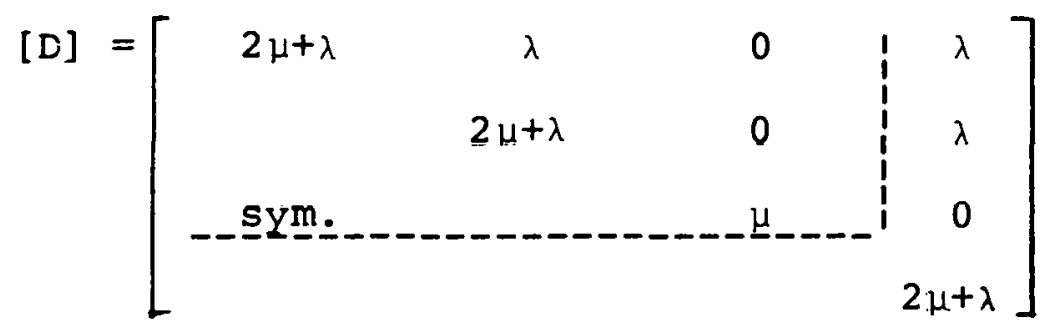

where the dashed lines identify the usual plane strain matrix for elastic problems.

The vector $\underset{\sim}{d}$ from Eq (C.8) is given by 
$\underset{\sim}{d}=\left\{\begin{array}{l}d_{1} \\ d_{2} \\ d_{3} \\ d_{4}\end{array}\right\}=\left[\begin{array}{l}(2 \mu+\lambda) a_{1}+\lambda\left(a_{2}+a_{4}\right) \\ \lambda a_{1}+(2 \mu+\lambda) a_{2}+\lambda a_{4} \\ \mu a_{3} \\ \lambda a_{1}+\lambda a_{2}+(2 \mu+\lambda) a_{4}\end{array}\right]=\left\{\begin{array}{l}2 \mu \\ a_{2} \\ \frac{a_{3}}{2} \\ a_{4}\end{array}\right\}+\left\{\begin{array}{l}a_{1} \\ 1 \\ 0 \\ 1\end{array}\right\}(c .17)=$

where $M=\lambda\left(a_{1}+a_{2}+a_{4}\right)$

Hence Eq (C.10) yields

$$
\gamma=h+2 \mu\left(a_{1}^{2}+a_{1}^{2}+\frac{1}{2} a_{3}^{2}+a_{4}^{2}\right)+M\left(a_{1}+a_{2}+a_{4}\right)
$$

and $\mathrm{Eq}$ (C.4) is given by 


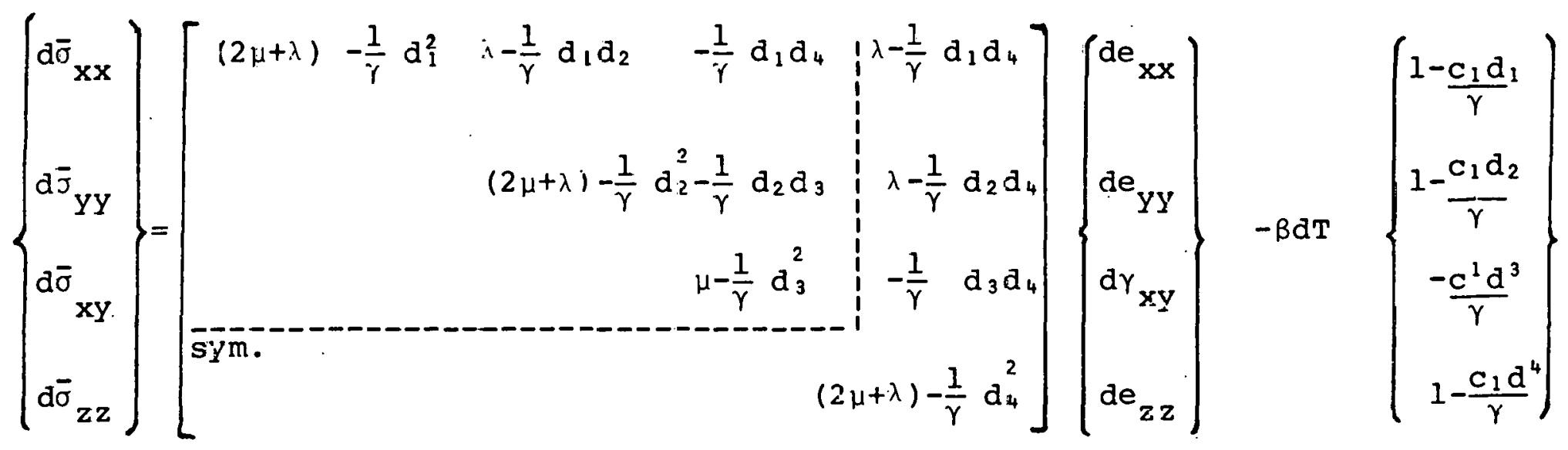

$\stackrel{1}{N}$

Using de ${ }_{z z}=$ zero for plane $\operatorname{strain}$ Eq (C.21) becomes

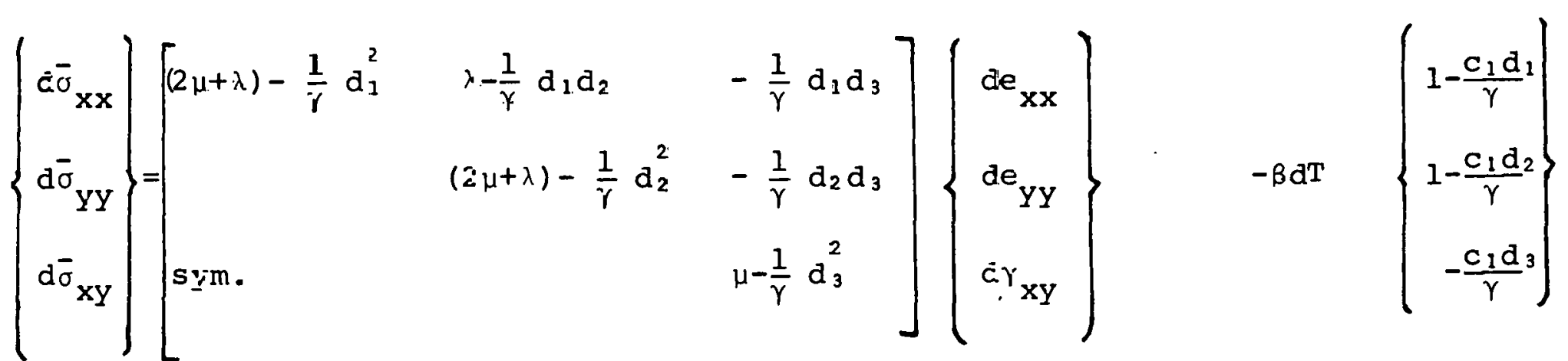

and

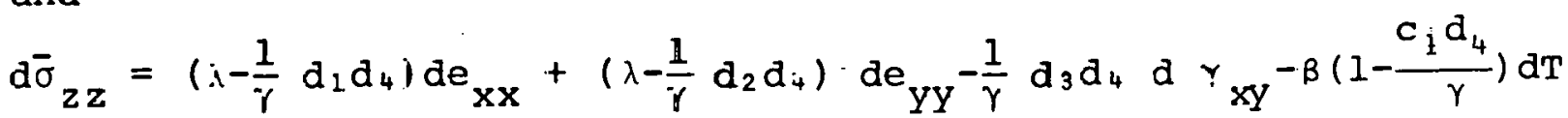


APPENDIX D

THE HARDENING FUNCTION, h

\section{D.I) Hardening Rule}

The hardening rule defines the motion of subsequent yield surfaces during plastic loading. For a perfectly plastic material, the yield stress does not depend on the degree of plastification. If the subsequent yield surfaces are a uniform expansion of the original yield curve, without translation, then the hardening rule is said to be isotropic. On the other hand, if the subsequent yield surfaces preserve their shape and orientation but translate in the stress space as a rigid body, the model is said to exhibit kinematic hardening. A combination of these two types of hardening results in the mixed hardening rule.

In this investigation, isotropic hardening is assumed with the four yield criteria cited in Appendix A. The yield criteria of Singh [60], (Appendix B), in which the elliptic cap moves and expands exhibits by definition mixed hardening.

In this Appendix, calculations of the hardening function, $h$, for an isotropic hardening model are presented. The calculation for $h$ ueing the model by singh [60] is presented in Appendix B. 


\section{D.2) The Hardening Function}

The hardening function, $h$, can be obtained by assuming that the slope of the stress-plastic strain curve for a uniaxial state of stress is the same as that for the multiaxial state case. This assumption can be achieved by appropriate definition of the equivalent stress and equivalent plastic strain, which reduces to the uniaxial case. The assumption is

$$
\frac{d \sigma}{d c_{p}}=H
$$

where $\sigma_{e}$ is the equivalent stress, $e_{p}$ is the equivalent plastic strain and $H$ is the slope of the uniaxial stressplastic strain, i.e.

$$
H=\frac{d \sigma x x}{d e_{x x}^{p}}
$$

where subscripts $x x$ refer to the uniaxial state of stress. The quantity $f\left(\sigma_{i j}\right)$ in $\mathrm{Eq.}(\mathrm{A.2})$ is commonly used to define of $[13,31]$ in the form

$$
f\left(\sigma_{i, j}\right)=c \sigma_{e}^{n}
$$

nr

$$
\sigma_{e}=\left[\frac{1}{c} f\right]^{1 / n}
$$

The definition of equivalent plastic strain, however, is not that simple since there are two definitions for $k$, Eqs. (3.3) and (3.5) using either the strain hardening or work hardening hypotheses. 
The strain hardening hypothesis leads to Eq.(3.6), i.e.

$$
d e_{p}=c\left(d e_{i j}^{p} d e_{i j}^{p}\right)^{1 / 2}
$$

The work hardening hypothesis, Eq. (3.4), can be written as

$$
d \omega_{p}=\bar{\sigma}_{i j} d e_{i j}^{p}
$$

Substituting from Eq.(3.19) into Eq.(D.6) gives

$$
d \omega_{p}=\bar{\sigma}_{i j} d \lambda \frac{\partial f}{\partial \bar{\sigma}}
$$

Since $f$ is homogeneous of degree $n$ in the stress, then by Euler's theorem

$$
d \omega_{p}=d \lambda n f
$$

By rewriting Eq. (D.6) as

$$
{ }^{d \omega_{p}}=\sigma e^{d e_{p}}
$$

the equivalent plastic increment can be defined as

$$
\mathrm{de}_{\mathrm{p}}=\frac{\mathrm{nf}}{\sigma_{\mathrm{e}}} \mathrm{d} \lambda
$$

For isotropic hardening Eqs. (3.1) and (3.2) reduce to

$$
F\left(\sigma_{i j}, k, T\right)=f\left(\sigma_{i j}\right)-\sigma_{o}(k, T)=0
$$

and hence Eq. (3.17) reduces to

$$
h=-\frac{\partial F}{\partial k} \bar{\sigma}_{i j} \frac{\partial F}{\partial \sigma_{i j}}
$$

i.e.

$$
h=-\frac{\partial F}{\partial k} \frac{1}{d \lambda}-d k
$$

Assuming an isothermal yield surface, Eq.(D.10) gives 


$$
\frac{\partial F}{\partial k}=-\frac{d \sigma_{o}}{d k}
$$

Hence Eq. (D.12) reduces to

$$
h=\frac{d \sigma_{0}}{d \lambda}
$$

Substituting Eqs.(D.9) and (D.10) into Eq.(D.14), we have

$$
h=\frac{d f}{d e_{p}} \frac{\sigma_{e}}{n f}
$$

Eq. (D.15) can be rewritten as

$$
h=\frac{d f}{d \sigma_{e}} \frac{d \sigma_{e}}{d e_{p}} \frac{\sigma_{e}}{n f}
$$

Substituting from Eq.(D.1) into Eq.(D.16) gives

$$
h=\frac{d f}{d \sigma_{e}} H \frac{\sigma_{e}}{n f}
$$

For $f=\sigma_{e}$ i.e., $n=1$, we have $h=H$. 


\section{APPENDIX E}

Numerical Procedure for Evaluating Stress Increment

\section{E.1 Introduction}

In this Appendix, the steps of using the incremental iterative scheme discussed in Chapter III are illustrated.

\section{E.2 Stress Increment}

For each element, stresses are assigned at its Gaussian points. During load application, plastic flow may commence at some Gaussian points, creating partially yielded or totally yielded elements. A procedure is designed in Ref. $[37,40]$ and is used in the code PCONSL, to determine the plastic portions and uses the appropriate constitutive relations to evaluate stress increment and corresponding residual loads. In this procedure, the following steps are repeated for every rth iteration and at every Gaussian point. (1) Incremental displacement and pore pressure are obtained by solving Eq.(3.63) in terms of the applied residual loads. Accordingly, the incremental strains are given by

$$
\Delta e_{\sim}^{r}=N_{e} \Delta{\underset{\sim}{u}}^{r}
$$

(2) Assuming elastic behavior, the elastic stress increment is 


$$
\Delta \underline{\sigma}^{r}=[D] \Delta e^{r}
$$

Hence the elastic stress vector

$$
\bar{\sigma}_{E}^{r}=\bar{\sigma}^{r-1}+\Delta \bar{\sigma}_{E}^{r}
$$

(3) The uniaxial yield stress is updated according to the hardening rule used. For the cap model, the procedure in Appendix $B$ is used. While, for the isotropic model, the yield stress is given by

$$
u_{0}^{r}=u_{u}+H e_{u}^{r-1}
$$

where $H$ and $e_{p}$ are given in Appendix $D$.

(4) Check the yield condition. The following flow chart is used to check whether yield starts this iteration or the point is previously yielded.

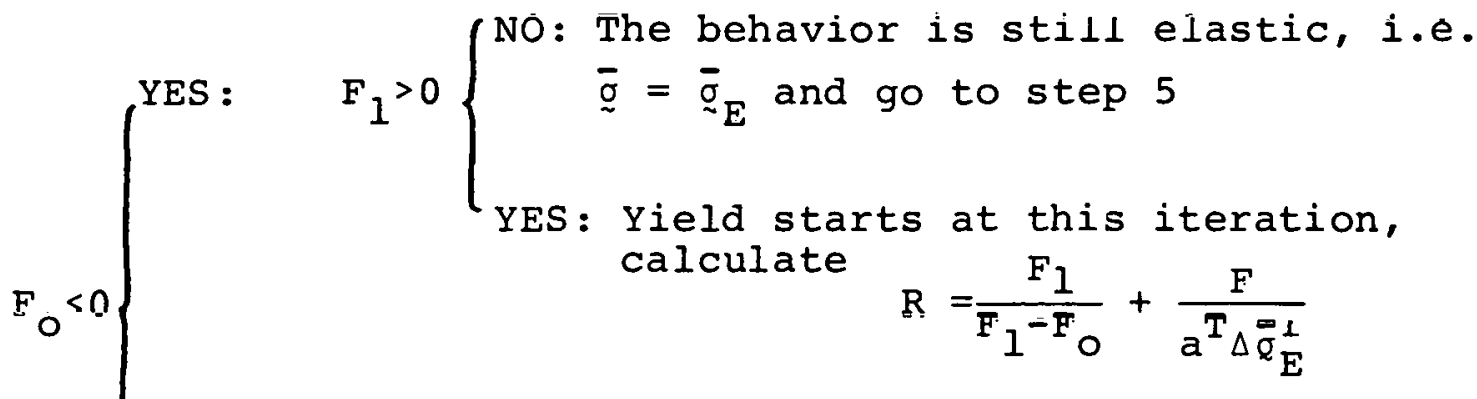

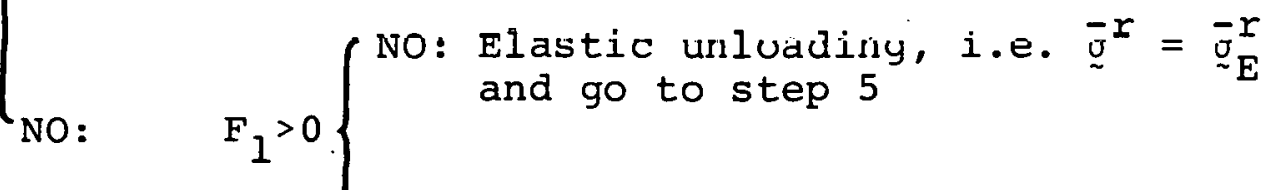

$$
\begin{aligned}
& \text { YES: Plastic flow continues, set } R=1
\end{aligned}
$$


where

$$
\begin{aligned}
& F_{0}=f\left(\bar{\sigma}^{r-1}\right)-\sigma_{0}^{r-1} \\
& F_{1}=f\left(\bar{\sigma}_{E}^{r}\right)-\sigma_{0}^{r-1} \\
& F=f\left(\underline{\tau} \frac{r}{l}\right)-\sigma_{0}^{r-1} \\
& \stackrel{\tau}{\tau}_{1}^{r}=\bar{\sigma}^{r-1}-\frac{F_{o}}{F_{1}-F_{O}} \Delta{\underset{\sim}{\sigma}}_{E}^{r} \\
& \stackrel{a}{a}^{r}=\frac{\partial F}{\partial \underset{\sim}{2} \underset{r}{r}}
\end{aligned}
$$

in which $F$ is the yield function. The condition $F_{0}<0$ and $F_{1}>0$ indicates a transition from elastic to plastic states occurs during this iteration. The reduction factor (1-R) is obtained above from the condition that $F=0$ at the contact state. Thus the trial elastic stress increment is subdivided into two parts. A stress state where the yield surface is contacted $\underline{\tau}^{r}$ given by

$$
\tau_{\sim}^{r}=\bar{\sim}^{r-1}+(1-B) \Delta \bar{\sigma}_{\tilde{E}}^{r}
$$

and a remaining part $\mathrm{R} \Delta \underset{\sim}{\sigma} \underset{\mathrm{E}}{\mathrm{r}}$ outside the yield surface. This part has to be eliminated by allowing plastic deformation to occur,

$$
\Delta{\underset{\sim}{\sigma}}^{r}=\Delta \bar{\sigma}_{E}^{r}-d \lambda[D] \underline{\sim}^{r}
$$

where $\underset{\sim}{a}=\frac{\partial F}{\partial \underset{\sim}{r}}$ and $d \lambda$ is given by Eq. (3.41). Figures (E.I) and (E.2) illustrate the incremental stress change in an already yielded point and a point at initial yield. 
It can be seen from these figures that even for a finite sized stress increment, the final stress point, D, may depart from the yield surface. This error can be eliminated by scaling the point, D, back to the yield surface, i.e.

$$
\bar{\sigma}^{r}=\bar{q}^{r} \times\left(\frac{\text { current yield stress }}{\sigma_{e}^{r}}\right)
$$

where $\sigma_{e}^{r}$ is the equivalent stress, (Appendix D). This method is known as the tangent stiffness method with radial relurn, Ref.[29,50]. The effective plastic strain is obtained using the work hardening hypothesis, as

$$
e_{p}^{r}=e_{p}^{r-1}+\frac{d \lambda a_{e}^{r} \sigma^{r}}{\sigma_{e}^{r}}
$$

(5) The residual loads are obtained using Eqs.(3.64) and $(3.65)$

\section{E.3 Refined Process for Scaling Stress Point to the Yield Siurface}

For relatively large stress increment, the scaling process, illustrated in Figs.(E.1) and (E.2), can lead to inaccurate results particularly if the point $D$ lies in the vicinity of a region of large curvature, Ref. [40].

In Refs. $[45,29]$ a secant stiffness method is presented for perfectly plastic von Mises material in lieu of the tangent stiffness method with radial return. The method is illustrated in Fig.(E.3), in which an intermediate state $\underline{s}^{r}$ is found as 


$$
\underline{\sim}^{r}=\frac{1}{2}\left({\underset{\sim}{E}}_{E}^{r}+\stackrel{\tau}{I}^{r}\right)
$$

and the flow vector is given as

$$
\stackrel{a}{r}^{r}=\frac{\partial F}{\partial S^{r}}
$$

then Eq.(E.II) is used to find the stress increment. It has been proved in Ref.[45] that the final state is exactly on the yield surface. However this method is not used in the present analysis since it is applicable only to perfectly plastic solids.

In Refs. $[37,40]$ a refined process is developed in using the tangent stiffness method with radial returns by relaxing the excess stress to the yield surface in several stages. The stress scaling can be performed after each stage or after the final stage only, Fig.(E.4). As the number of steps. increases, the accuracy increased and also the cost.

\section{E.4 Stiffness Update}

It was mentioned in Chapter III that the stiffness matrix has to be updated, otherwise excessive iterations are required. The stiffness is changed whenever $e_{p}>0$. This change can be performed in any iteration during the increment. It is recommended in Ret.[40] to re-evaluate the stiffness at the second iteration to increase the rate of convergence. 


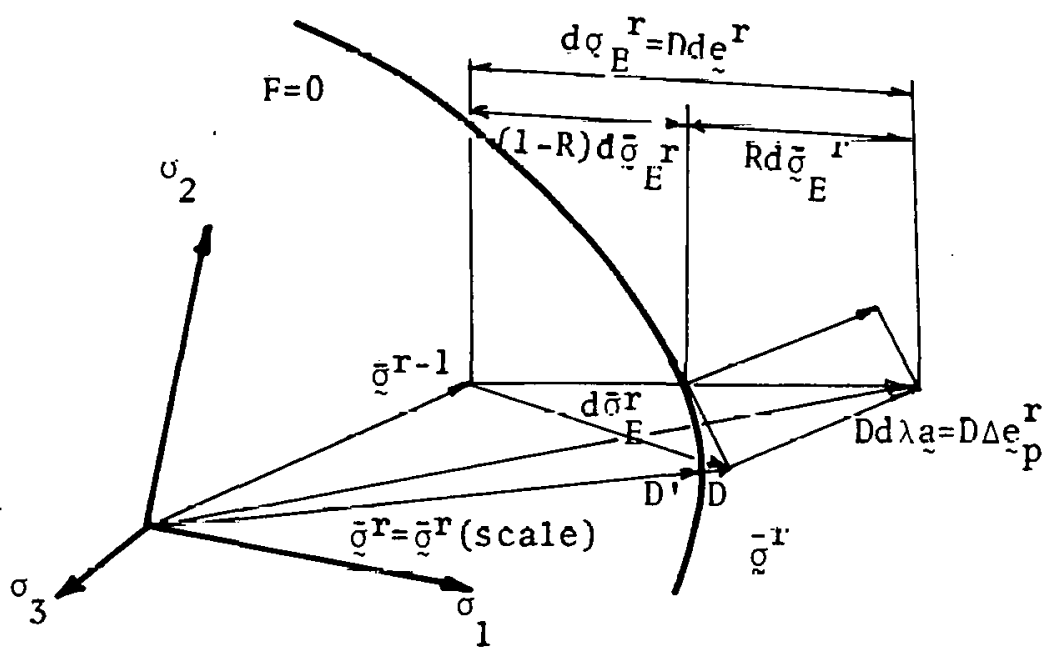

Figure (E. 1): Incremental Stress Changes at a Point in an Elasto-Plastic Continuum at Initial Yield 


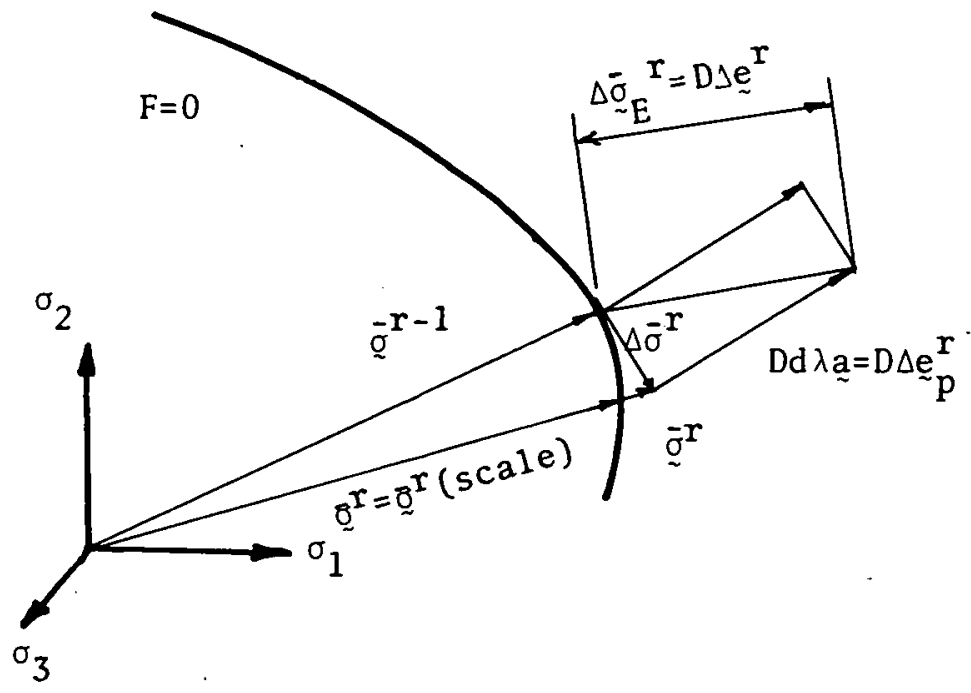

Pigure (E.2): Incremencal Stress Changes in an Already Yielded foint in an Elasto-Plastic Continuum 


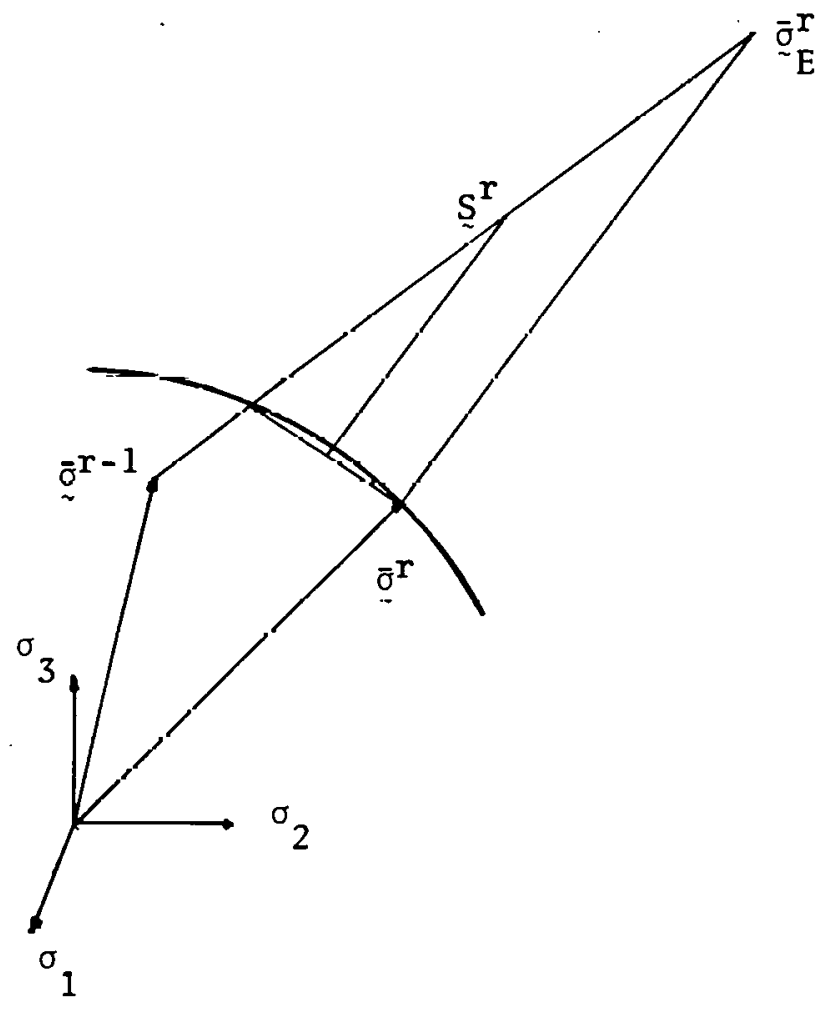

Figure (E.3): Secant Stiffness Method 


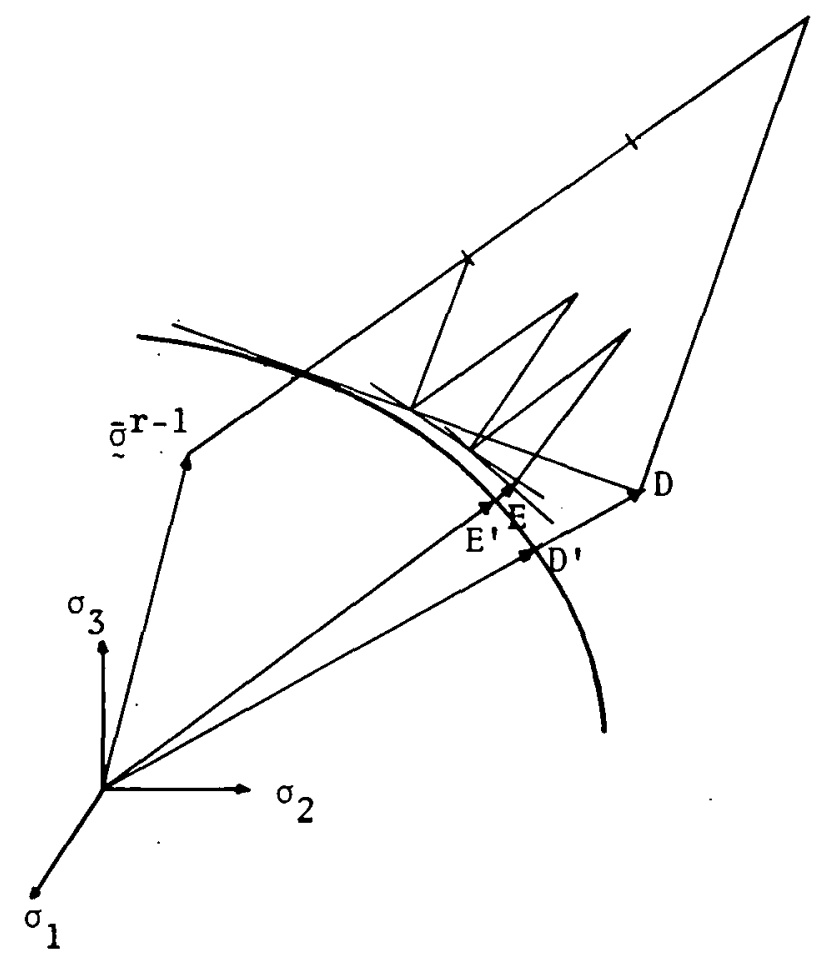

Figure (E.4): Multi-step Tangent Stiffness Method with Scaling 


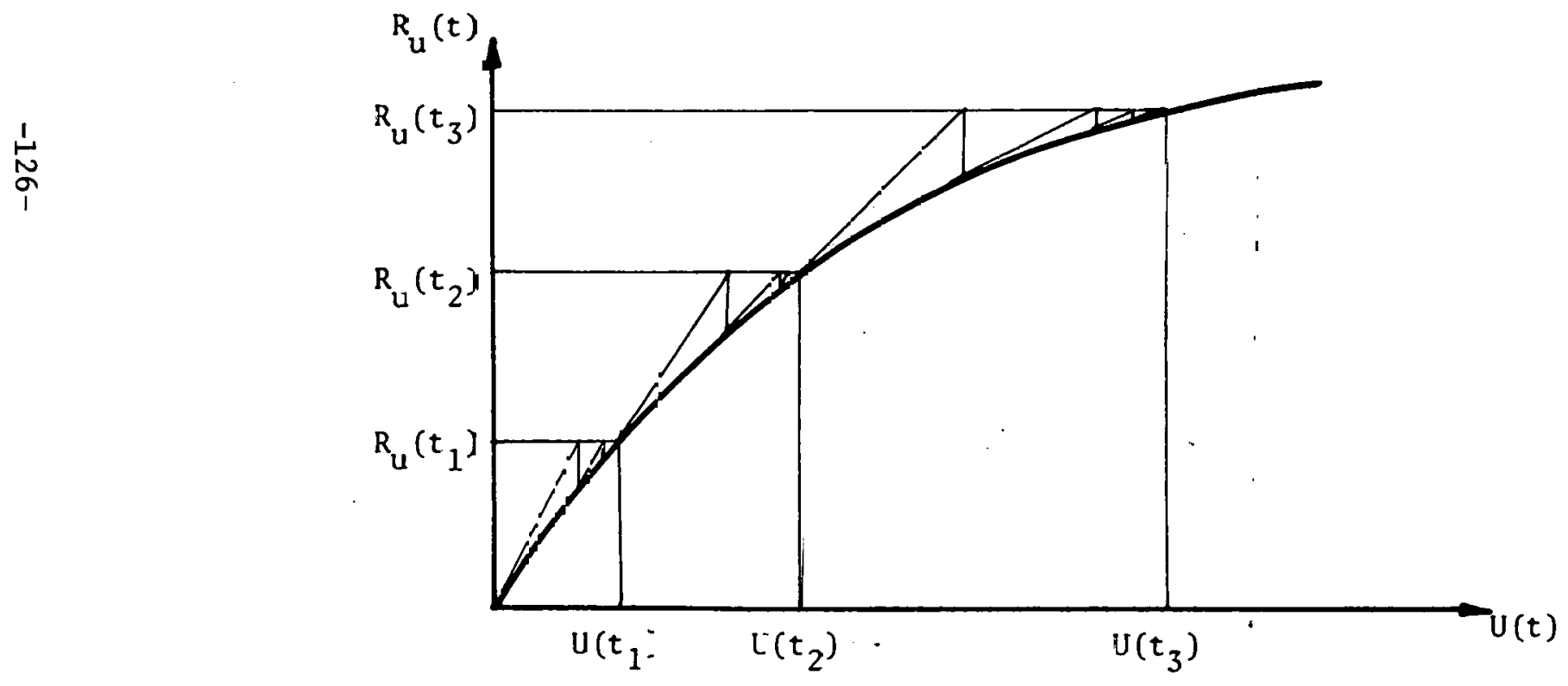

Figure (E.5): The Incremental Iterative Scheme 


\section{APPENDIX F}

\section{PROOF OF THEOREM I}

To prove this theorem we let

$$
\bar{\omega}=\left\{\bar{u}_{i}, \bar{e}_{i j}, \bar{\sigma}_{i j}, \bar{\pi}, \bar{q}_{i}, \bar{\theta}_{i}, \bar{T}, \bar{h}_{i}, \bar{\phi}_{i}\right\}
$$

Then

$$
\begin{aligned}
& \Delta \bar{\omega} J_{l}(\omega)=\int_{R}-\bar{u}_{i} *\left[\left(\sigma_{i j}+\pi \delta_{i j}-\beta T \delta_{i j}\right)_{, j}+2 \rho f_{i}\right] d R \\
& +\int_{R} \sigma_{i j} * \bar{u}_{(i, j)} d R+\int_{R} \pi * \bar{u}_{i, i} d R-\int_{R} T * B \bar{u}_{i, i} d R \\
& +\int_{S_{2}} \bar{u}_{i} *\left[\left(\sigma_{i j}+\pi \delta_{i j}-\beta T \delta_{i j}\right) n_{j}-2 t_{i}\right] d S \\
& -\int_{s_{1}}\left(\sigma_{i j}+\pi \delta_{i j}-\beta T \delta_{i j}\right) * n_{j} \bar{u}_{i} d s \\
& +\int_{R} \bar{e}_{i j}{ }^{*}\left[E_{i, j k l} e_{k l}-\sigma_{i j}\right] d R-\int_{R} u_{i}{ }^{*} \bar{\sigma}_{i j, j} d R-\int_{R} e_{i j}{ }^{* \bar{\sigma}_{i j}} d R \\
& +\int_{R} \bar{\sigma}_{i j}{ }^{*}\left(u_{(i, j)}-e_{i j}\right) d R+\int_{S_{2}} u_{i}{ }^{*} \bar{\sigma}_{i j} n_{j} d S \\
& -\int_{s_{1}} \bar{\sigma}_{i j}{ }^{*} n_{j}\left(u_{i}-2 \hat{u}_{i}\right) d s-\int_{R} u_{i}{ }^{*} \bar{\pi}, i d R-\int_{R} q_{i}{ }^{*} g^{\prime *} \bar{\pi}_{, i} d R \\
& +\int_{R} \bar{\pi} *\left[u_{i, i}+g^{\prime *} q_{i, i}\right] d R+\int_{s_{2}} u_{i}{ }^{* \bar{\pi} \delta}{ }_{i j} n_{j} d S \\
& -\int_{S_{1}} \bar{\pi} \delta_{i j}{ }^{*} n_{j}\left(u_{i}-2 u_{i}\right) d S+\int_{S_{3}} Q^{*} g^{\prime *} \bar{\pi} d S-\int_{S_{4}} \bar{\pi}^{*} g^{\prime *}(Q-2 \hat{Q}) d S
\end{aligned}
$$




$$
\begin{aligned}
& +\int_{R} q_{i}{ }^{*} g *\left(\theta_{i}-\pi, i^{-2 \rho}{ }_{2} f_{i}\right) d R+\int_{R} \pi * g{ }^{*} \bar{q}_{i, i} d R \\
& +\int_{S_{3}} \bar{q}_{i} n_{i}{ }^{*} g^{\prime *}(\pi-2 \hat{\pi}) d S-\int_{S_{4}} \pi * g^{\prime *} q_{i} n_{j} d S+\int_{R} q_{i}{ }^{*} g^{\prime *} \bar{\theta}_{i} d R \\
& +\int_{R} \bar{\theta}_{i}{ }^{*} g^{\prime *}\left(q_{i}-2 k_{i j}{ }^{\theta}\right) d R+\int_{R} u_{i}{ }^{*} \bar{T}, i d R+\int_{R} h_{i}{ }^{*} g^{\prime *} \bar{T}, i \frac{1}{\tau_{0}} d R \\
& -\int_{R} \bar{T}^{\star}\left[\beta u_{i, i}+\frac{1}{\tau_{0}} g^{\prime *} h_{i, i}+\frac{2 \rho C C_{v}}{T_{o}}\right] d R-\int_{S_{2}} u_{i} \star_{\beta} \bar{T} \delta_{i j} n_{j} d S \\
& +\int_{\varepsilon_{1}} B \overline{\mathrm{T}} \delta{ }_{i j}{ }^{\star} n_{j}\left(u_{i}-2 \hat{\mathrm{u}}_{i}\right) d S-\int_{S_{j}} H^{*} g^{\prime} * \overline{\mathrm{T}} \frac{\mathrm{l}}{\tau_{n}} \mathrm{dS} \\
& +\int_{R} \bar{h}_{i}{ }^{*} g^{\prime *}\left(T, i^{-\phi}{ }_{i}\right) \frac{1}{\tau_{0}} d R-\int_{R} \phi_{i}{ }^{*} g^{\prime} * \bar{h}_{i} \frac{1}{\tau_{0}} d R-\int_{R} T^{*} g^{\prime} * \bar{h}_{i, i} \frac{1}{\tau} d R \\
& -\int_{S_{5}} \bar{h}_{i^{\prime}} n_{i}{ }^{*} g^{\prime *}(T-2 \hat{T}) \frac{l}{\tau_{0}} d S+\int_{S_{6}} T^{*} g^{\prime *} \bar{h}_{i} n_{i} \frac{l}{\tau_{0}} d S-\int_{R} h_{i} g^{\prime *}{ }_{i} \frac{1}{0} d R \\
& -\int_{R} \bar{\phi}_{i}{ }^{*}\left(2 k_{i j}{ }^{*} \phi_{j}+g^{\prime *} h_{i}\right) \frac{l}{\tau_{0}} d R
\end{aligned}
$$

Substitution of Eqs.(2.68) through (2.72) into Eq.(F.1) $\because y i e l d s$

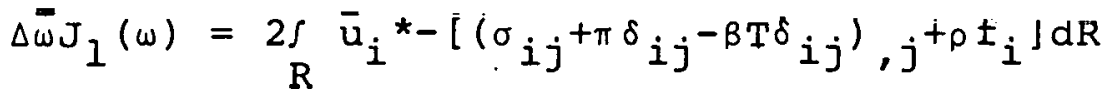

$$
\begin{aligned}
& +2 \int \bar{u}_{i} *\left[\left(\sigma_{i j}+\pi \delta_{i j}-\beta T \delta_{i j}\right) n_{j}-\dot{t}_{i}\right] d S \\
& +\underset{R}{2 \delta_{1 j}} \bar{e}_{1 j}\left[E_{i j k l} e_{k l}-\sigma_{i j}\right] d R+\underset{R}{2 \delta_{i j}} \bar{\sigma}_{i j}\left[u_{(i, j)}-e_{i j}\right] d R \\
& -2 S_{S_{1}} \bar{\sigma}_{i j}{ }^{*} n_{j}\left(u_{i}-\hat{u}_{i}\right) d s+2 f_{R} \bar{\pi} *\left(u_{i, i}+g^{\prime *} q_{i, i}\right) d R
\end{aligned}
$$




$$
\begin{aligned}
& -2 \int S_{1} \bar{\pi} \delta_{i j}{ }^{*} n_{j}\left(u_{i}-\hat{u}_{i}\right) d S-2 S_{S} \bar{\pi}^{*} g^{\prime *}(Q-\hat{Q}) d S \\
& +2 \int_{R} \bar{q}_{i}^{*} g^{\prime *}\left(\theta_{i}-\pi, i^{-\rho} f_{i}\right) d R+2 S_{S_{3}} \bar{q}_{i} n_{i}{ }^{*} g^{\prime *}(\pi-\hat{\pi}) d S \\
& +\underset{R}{2 \int} \bar{\theta}_{i}^{*} g^{\prime *}\left(q_{i}-K_{i j}{ }_{j}\right) d R-\underset{R}{2 \int} \bar{T} *\left[\beta u_{i, i}+\left(g^{\prime} * h_{i, i}+\rho C_{v} T / \tau o\right] d R\right. \\
& +\underset{S_{1}}{2 \int} \beta \bar{T} \delta_{i j}{ }^{*} n_{j}\left(u_{i}-\hat{u}_{i}\right) d S+\underset{S_{6}}{2 S} \bar{T}{ }^{\prime} g^{\prime *}(H-\hat{H}) \frac{l}{\tau_{0}} d S \\
& +\underset{R}{2 \int} \bar{h}_{i}{ }^{*} g^{\prime *}\left(T, i-\phi_{i}\right) \frac{l}{\tau_{0}} d R-2 \int \bar{h}_{i} n_{i}{ }^{*} g^{\prime *}(T-\hat{T}) d S \\
& -\underset{R}{2 \int} \bar{\phi}_{i}^{*} g^{\prime *}\left(h_{i}+k_{i j}{ }^{\phi} j\right) \frac{l}{\tau_{0}} d R
\end{aligned}
$$

If the field equations (2.32) through (2.40) together with the boundary conditions (2.41) through (2.48) are satisfied, the right hand side of Eq.(F.2) vanishes as one of the arguments of the bilinear map vanishes at the solution state. Conversely, if $\Delta_{\omega} J_{1}(\omega)$ vanishes for every smooth function $\bar{w}$, we choose, for example, $\bar{\omega}=\{\bar{u}, 0,0,0,0,0,0,0,0\}$ with $\vec{u}=0$ on $\mathrm{S}_{2} \times[0, \infty)$, then by the non-degenerate property of the bilinear map, for arbitrary $\bar{u}$ implies that Eq. (2.38) is obtained. Following the same procedure for the choice of $\bar{w}$ such that all but one term on the right hand side of Eq.(F.2) identically vanishes, implies the satisfaction of a field or boundary equation. Thus vanishing of $\Delta-J_{1}(\omega) \quad \bar{\omega} \in W$ implies the field Eqs.(2.32-2.40) together with the boundary conditions $(2.41-2.48)$. 